\title{
Process-related Impurities of Pazopanib
}

\section{Svetlana Terentjeva, Dzintra Muceniece and Viesturs Lüsis*}

Latvian Institute of Organic Synthesis, Riga, LV 1006, Latvia

\section{SUPPORTING INFORMATION}

NMR spectra (including ${ }^{1} \mathrm{H},{ }^{13} \mathrm{C}, \mathrm{COSY}$, HSQC, HMBC, NOESY) of obtained compounds.

$\begin{array}{llcl}\text { Compound } & \text { page } & \text { Compound } & \text { page } \\ \mathbf{3} & \text { S2- S4 } & \mathbf{1 6} & \text { S34- S37 } \\ \mathbf{4} & \text { S5- S7 } & \mathbf{1 7} & \text { S38- S41 } \\ \mathbf{8} & \text { S8- S11 } & \mathbf{1 8} & \text { S42- S47 } \\ \mathbf{9} & \text { S12- S15 } & \mathbf{1 9} & \text { S48, S49 } \\ \mathbf{1 0} & \text { S16- S19 } & \mathbf{2 1} & \text { S50- S53 } \\ \mathbf{1 2} & \text { S20- S23 } & \mathbf{2 2} & \text { S54- S57 } \\ \mathbf{1 3} & \text { S24- S28 } & \mathbf{2 3} & \text { S58- S61 } \\ \mathbf{1 4} & \text { S29- S31 } & \mathbf{2 5} & \text { S62- S65 }\end{array}$

$15 \quad$ S32, S33




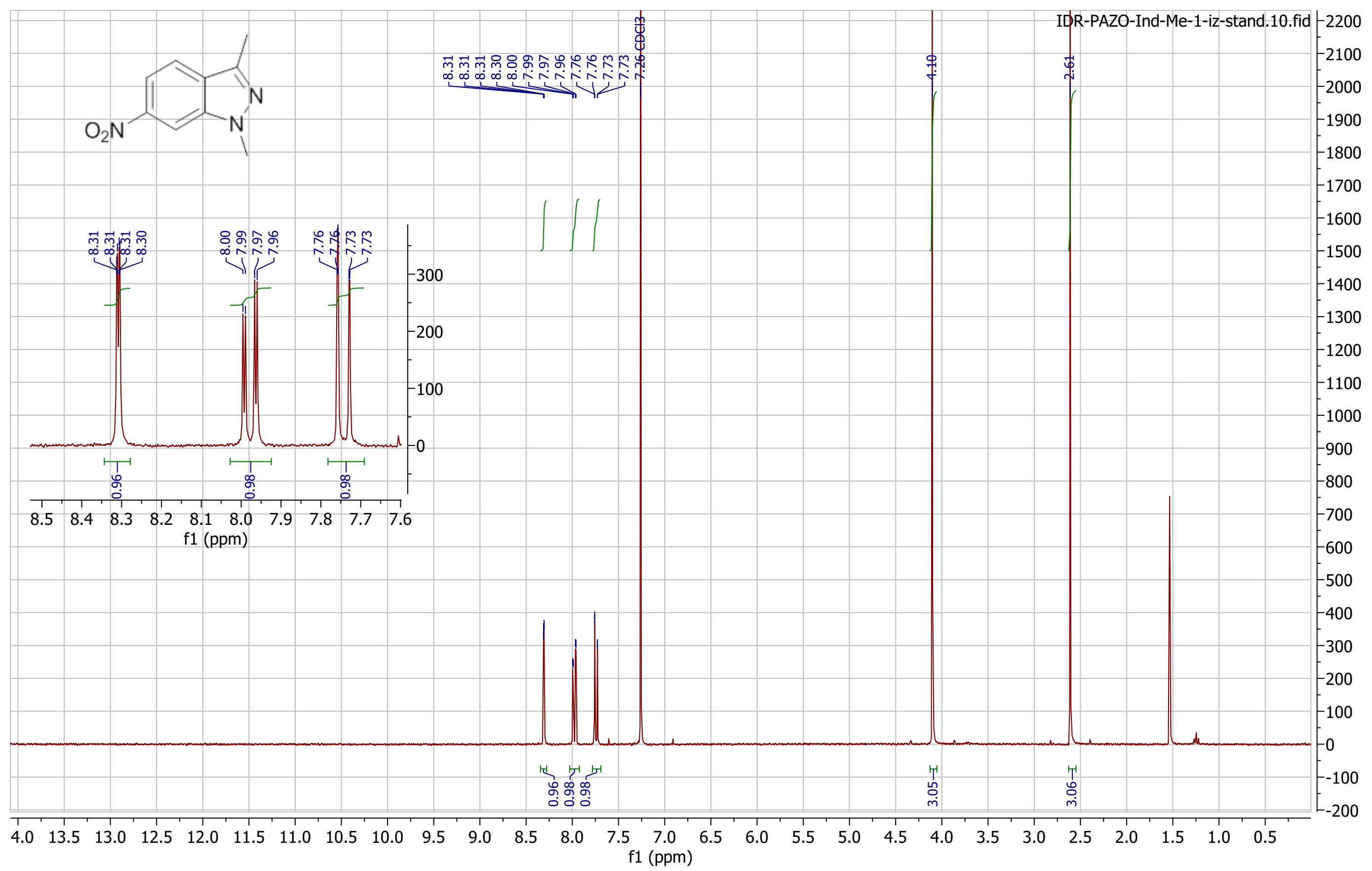

Fig. ${ }^{1} \mathrm{H}-\mathrm{NMR}$ spectrum (300 MHz, $\left.\mathrm{CDCl}_{3}\right)$ of 1,3-dimethyl-6-nitro- $1 H$-indazole (3) 


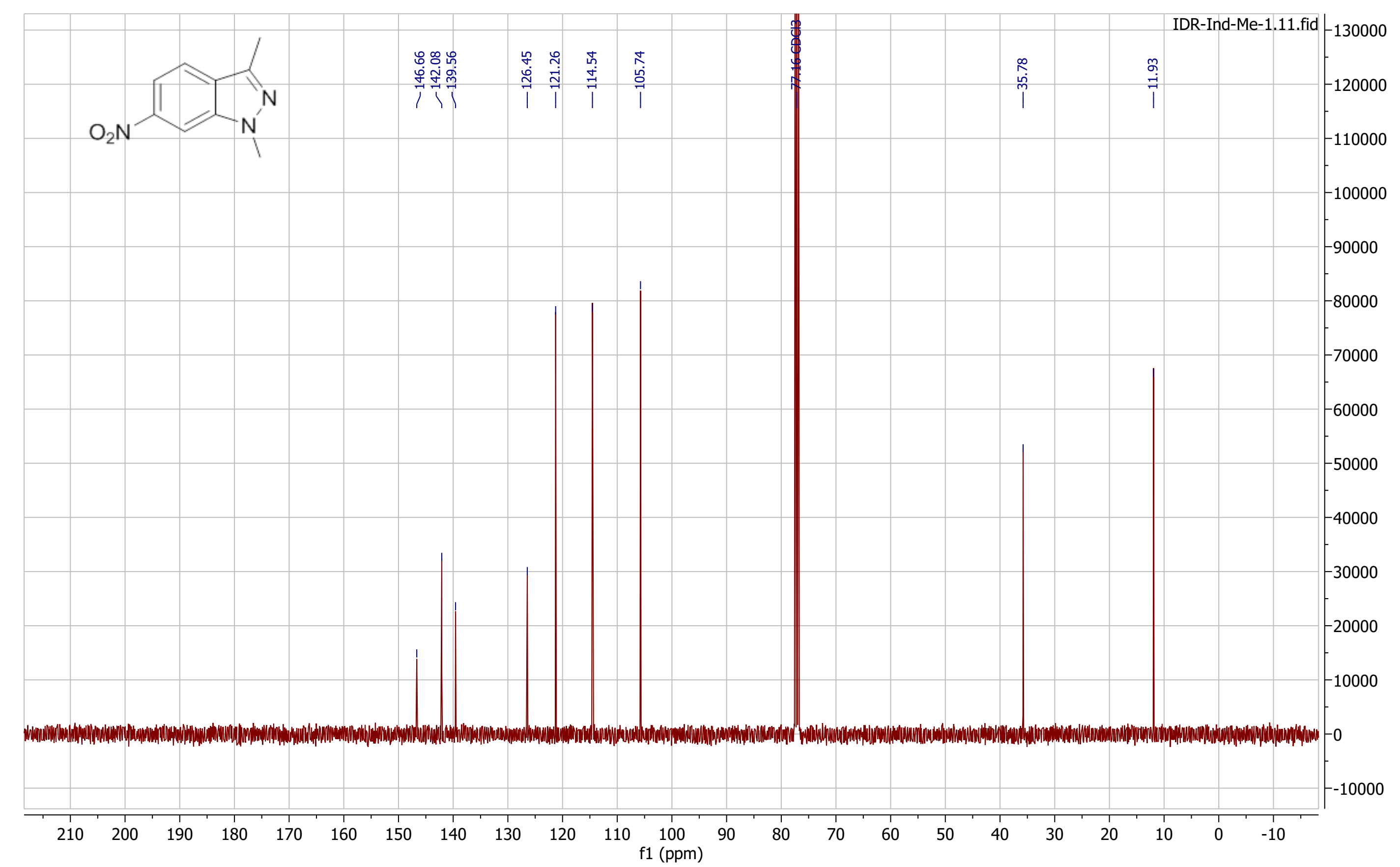

Fig. $2{ }^{13} \mathrm{C}$-NMR spectrum (100 MHz, $\left.\mathrm{CDCl}_{3}\right)$ of 1,3-dimethyl-6-nitro- $1 H$-indazole (3) 


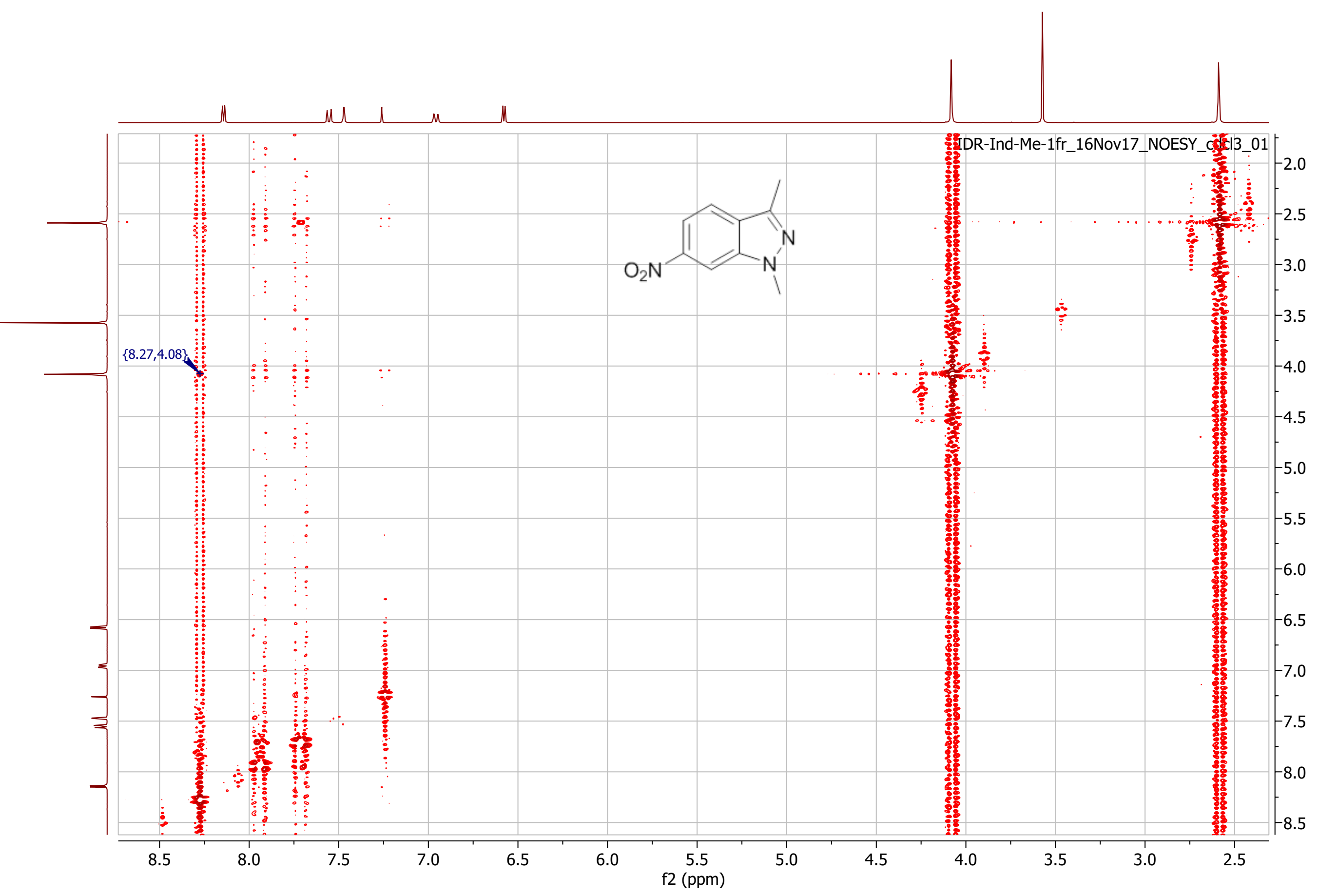

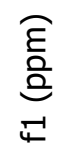

Fig.3 Part of NOESY spectrum (400 MHz, $\left.\mathrm{CDCl}_{3}\right)$ of 1,3-dimethyl-6-nitro- $1 H$-indazole (3) 


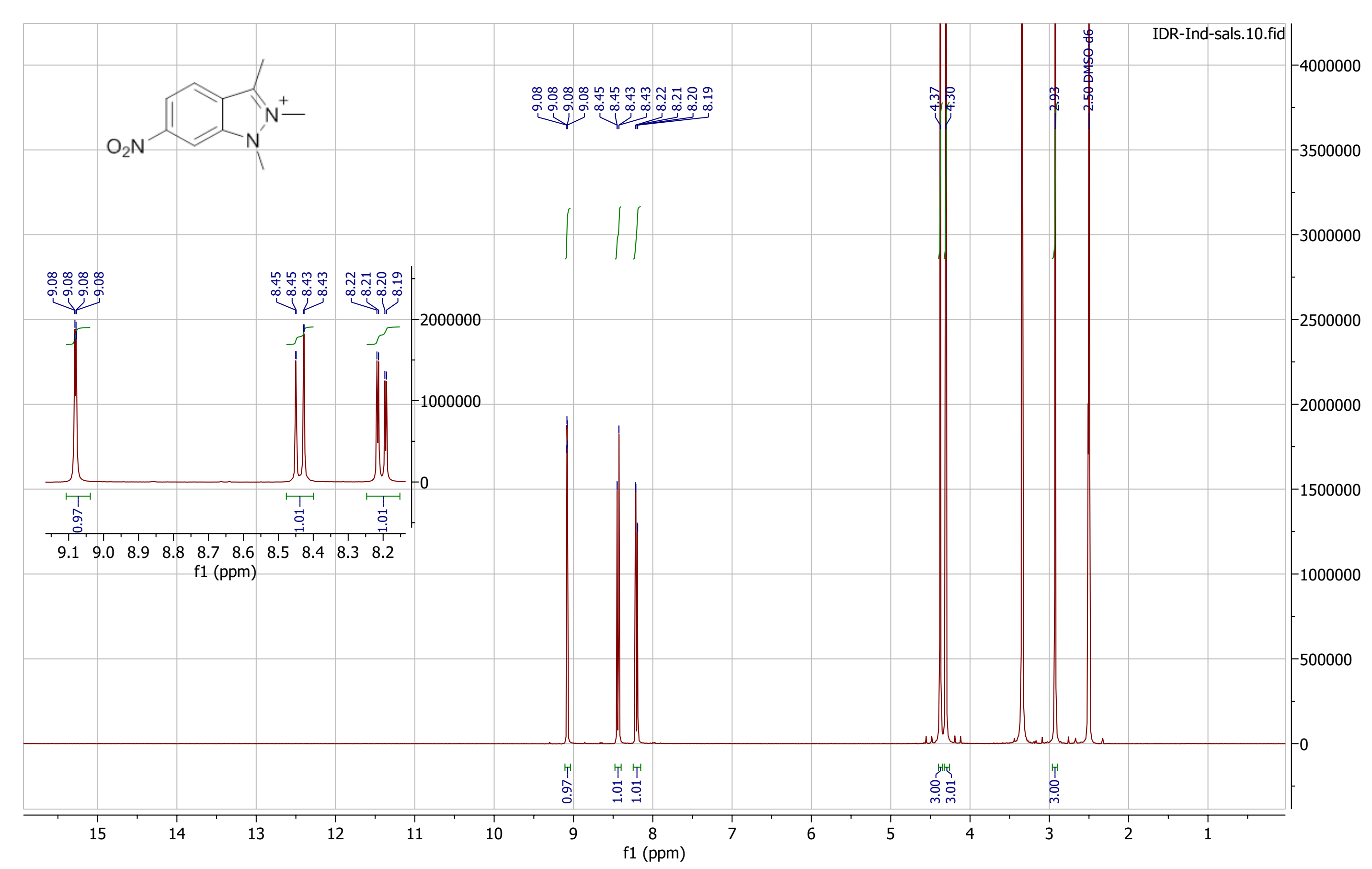

Fig. $4{ }^{1} \mathrm{H}$ NMRspectrum (300 MHz, DMSO-d 6 ) of 1,2,3-trimethyl-6-nitro- $1 H$-indazol-2-ium perchlorate (4-ClO4) 


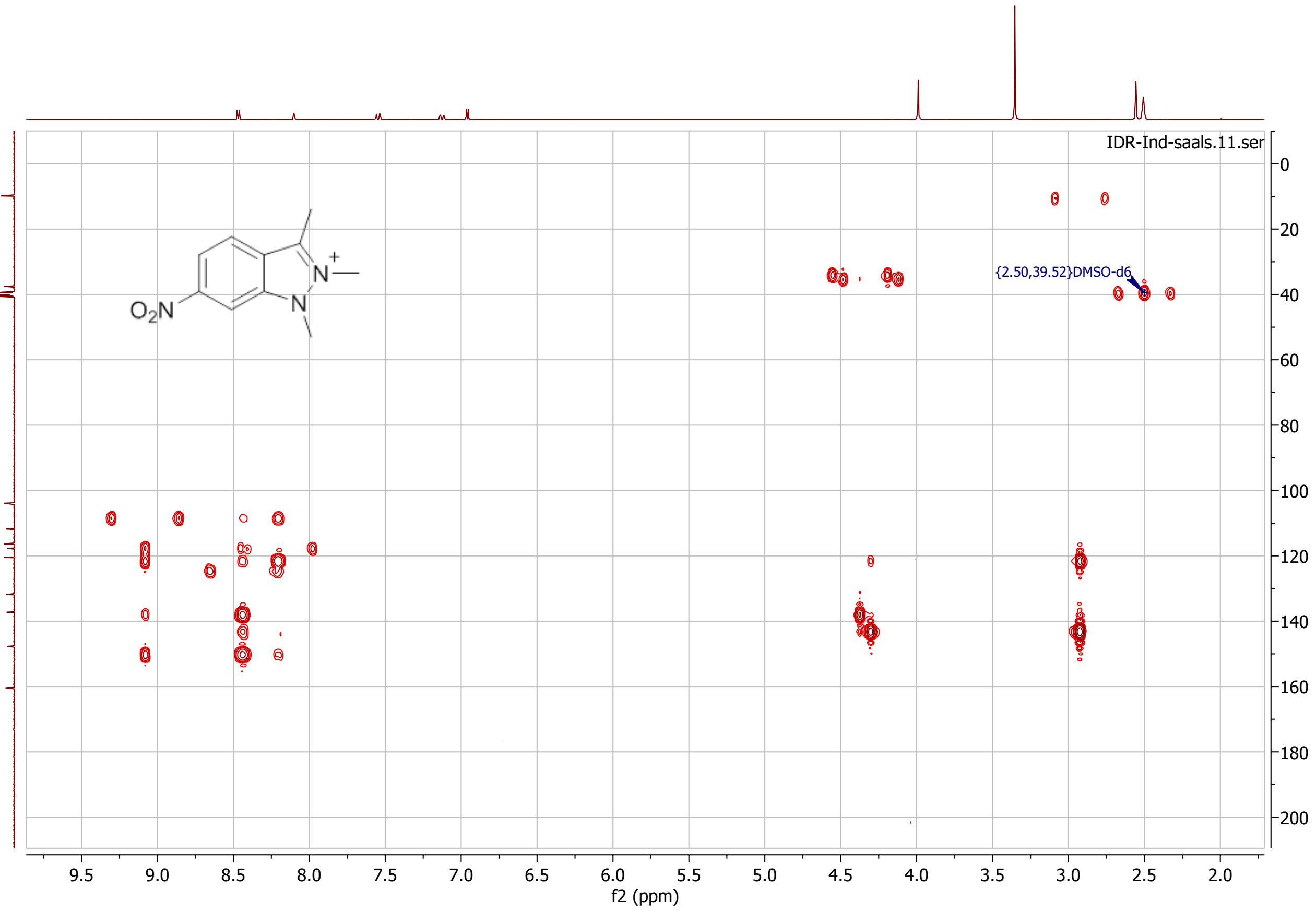

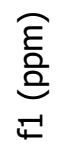

Fig.5 HMBC spectrum (400 MHz, $\left.100 \mathrm{MHz}, \mathrm{DMSO}-\mathrm{d}_{6}\right)$ of 1,2,3-trimethyl-6-nitro-1H-indazol-2-ium perchlorate $\left(\mathbf{4} \cdot \mathbf{C l O}_{4}\right)$ 


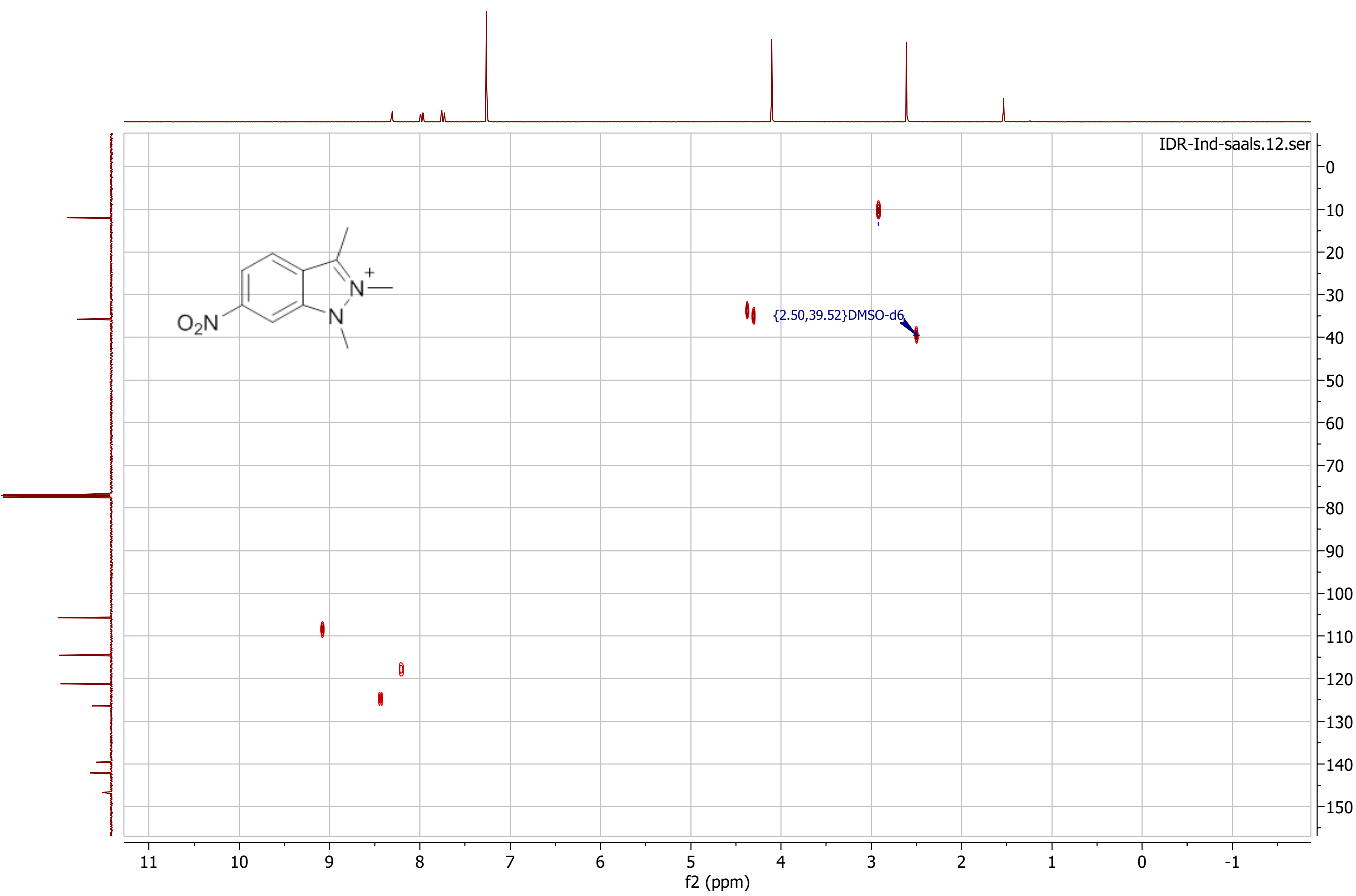

Fig.6 HSQC spectrum $\left(400 \mathrm{MHz}, 100 \mathrm{MHz}, \mathrm{DMSO}-\mathrm{d}_{6}\right)$ of 1,2,3-trimethyl-6-nitro-1H-indazol-2-ium perchlorate $\left(\mathbf{4} \cdot \mathbf{C l O}_{\mathbf{4}}\right)$ 

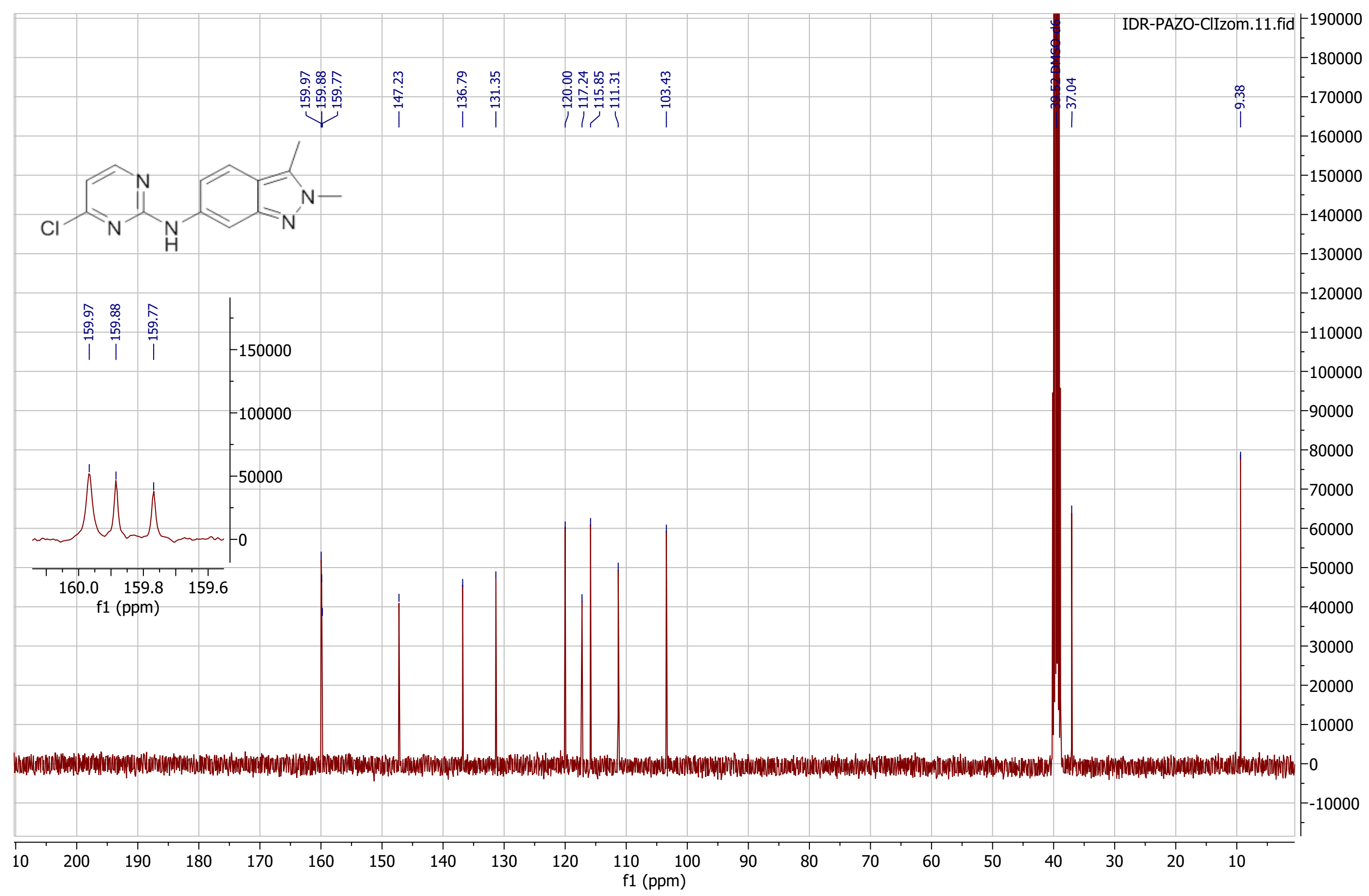

Fig. $8{ }^{13} \mathrm{C}-\mathrm{NMR}$ spectrum (100 MHz, DMSO-d $\mathrm{d}_{6}$ ) of (4-chloropyrimidin-2-yl)-(2,3-dimethyl-2H-indazol-6-yl)amine (8) 


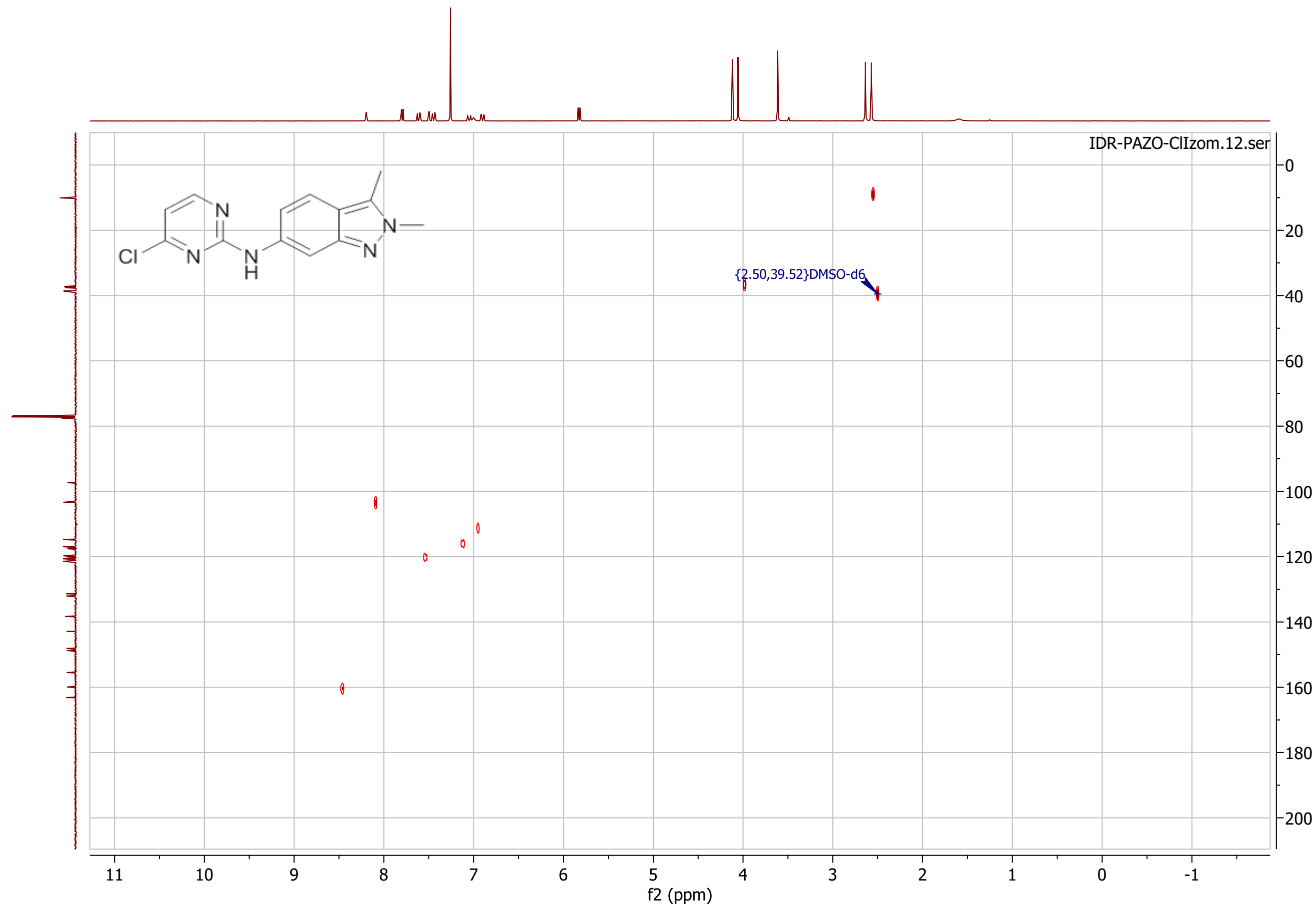

Fig.9 HSQC spectrum (400 MHz, $100 \mathrm{MHz}$, DMSO-d 6 ) of (4-chloropyrimidin-2-yl)-(2,3-dimethyl-2H-indazol-6-yl)amine (8) 


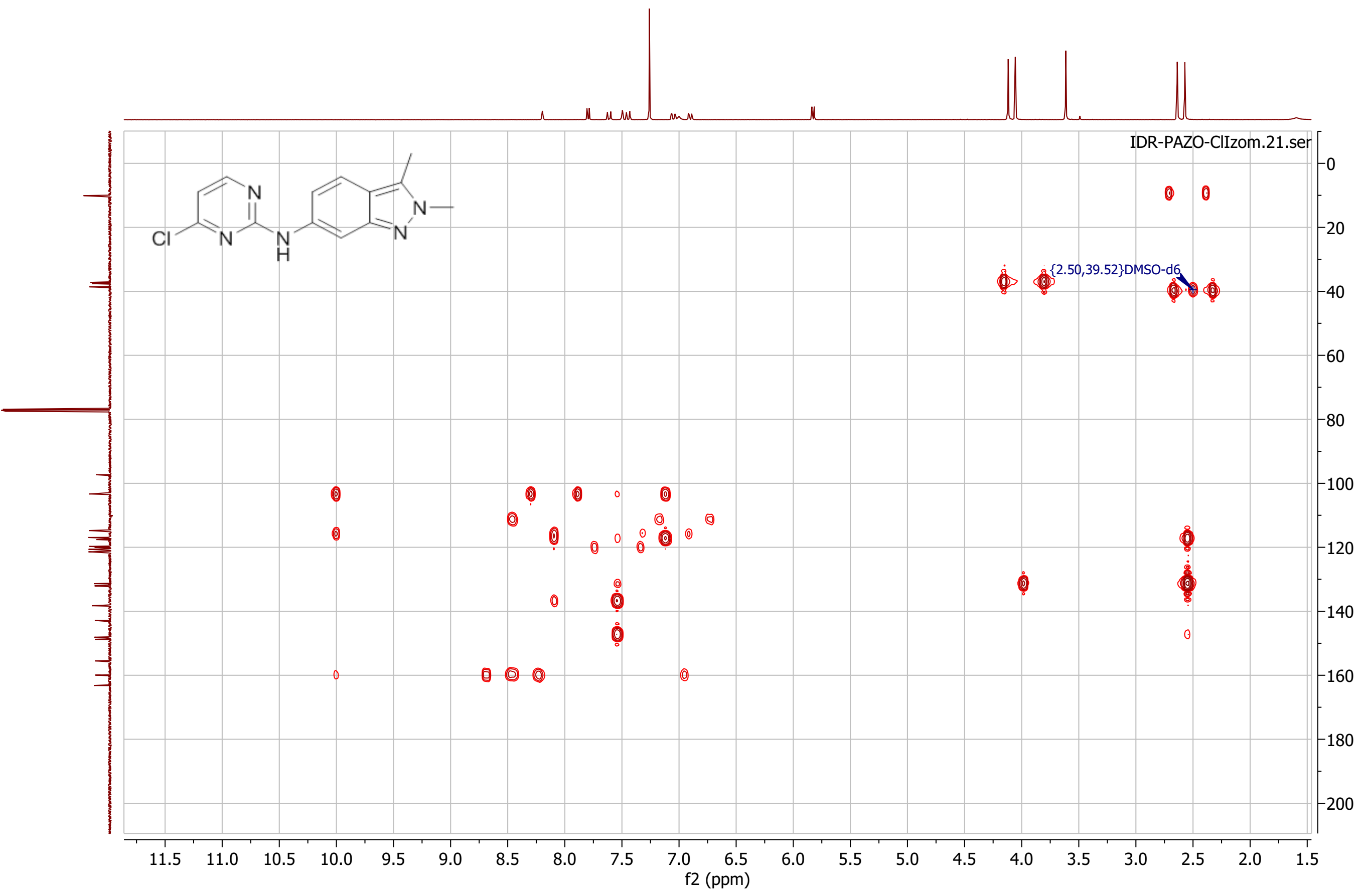

Fig.10 HMBC spectrum (400 MHz, 100 MHz, DMSO-d ) of (4-chloropyrimidin-2-yl)-(2,3-dimethyl-2H-indazol-6-yl)amine (8) 


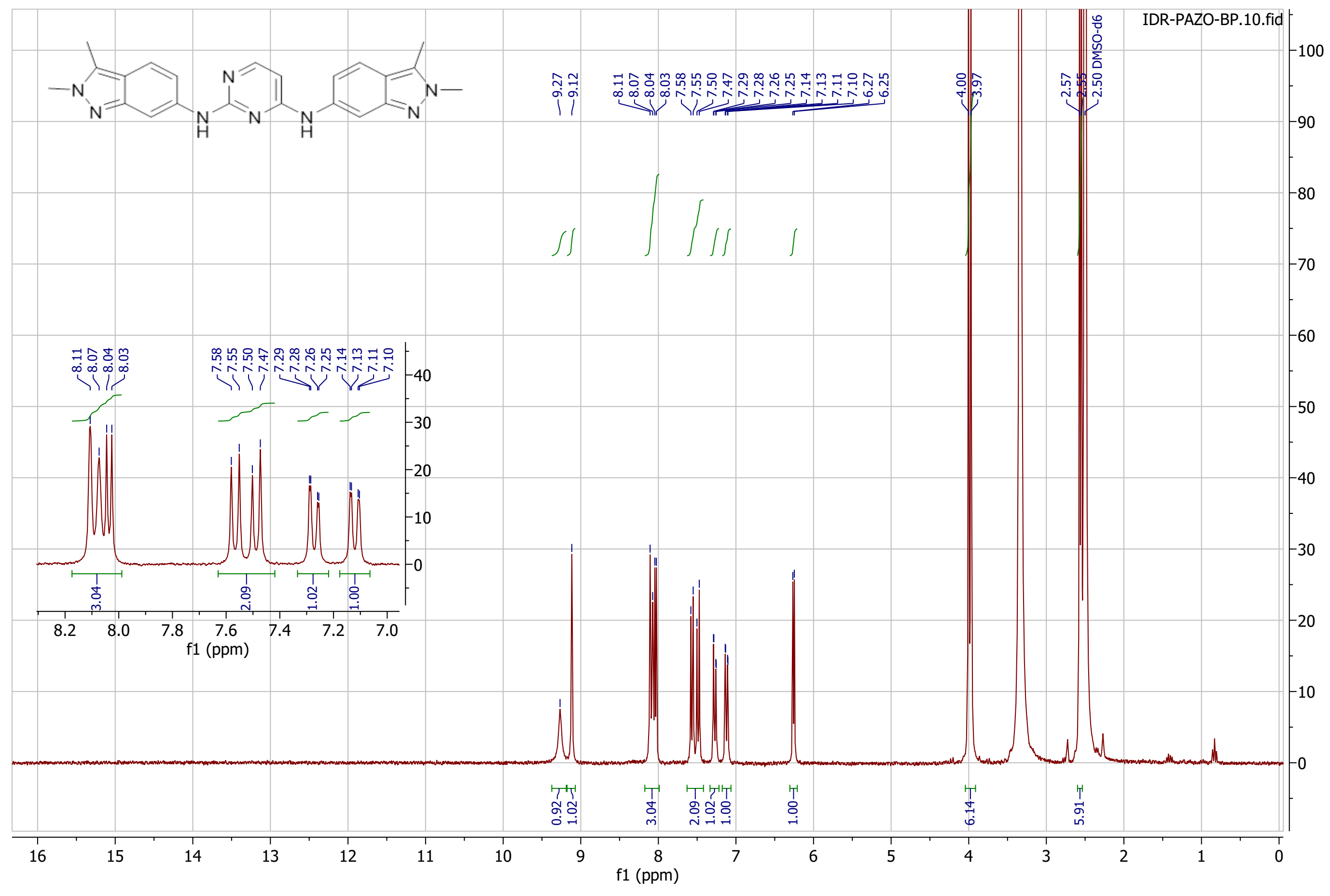

Fig. $11{ }^{1} \mathrm{H}$ NMR specturm (300 MHz, DMSO-d 6 ) of $N^{2}, N^{4}$-bis-(2,3-dimethyl-2H-indazol-6-yl)-pyrimidine-2,4-diamine (9) 


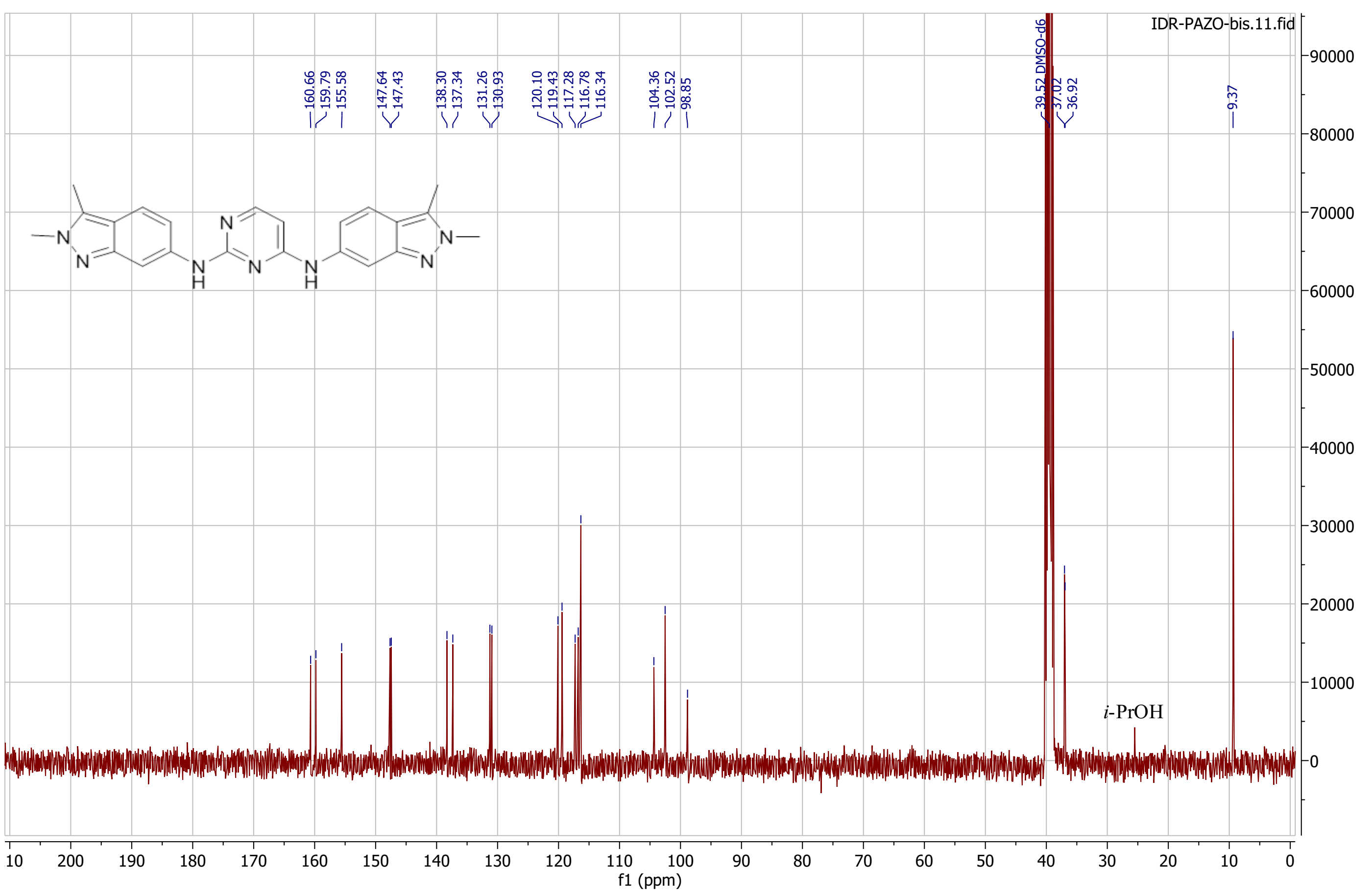

Fig.12 ${ }^{13} \mathrm{C}$ NMR spectrum (100 MHz, DMSO-d 6 ) of $N^{2}, N^{4}$-bis-(2,3-dimethyl-2 $H$-indazol-6-yl)-pyrimidine-2,4-diamine (9) 


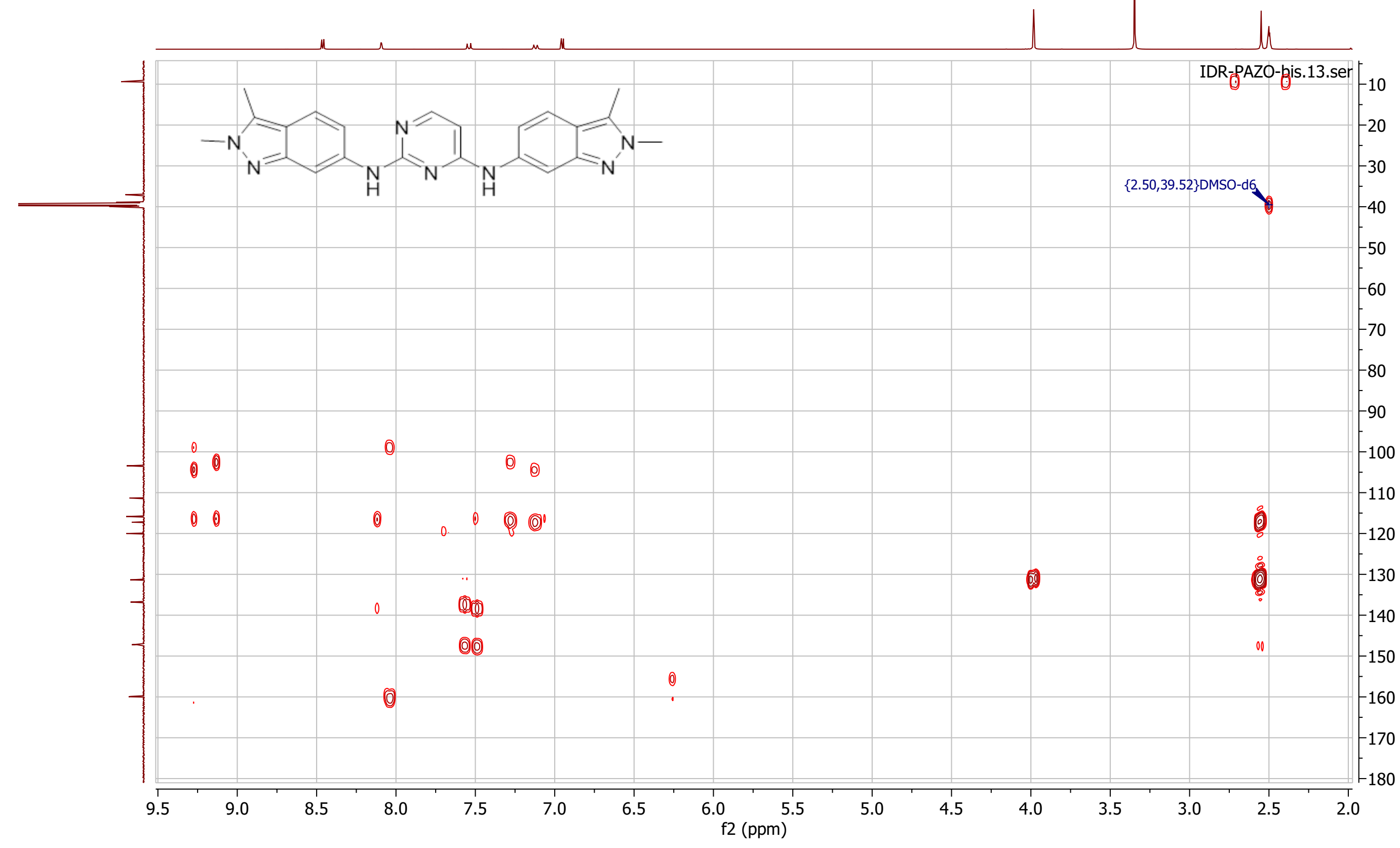

Fig.13 HMBC spectrum (400 MHz, $\left.100 \mathrm{MHz}, \mathrm{DMSO}-\mathrm{d}_{6}\right)$ of $N^{2}, N^{4}$-bis-(2,3-dimethyl-2H-indazol-6-yl)-pyrimidine-2,4-diamine (9) 


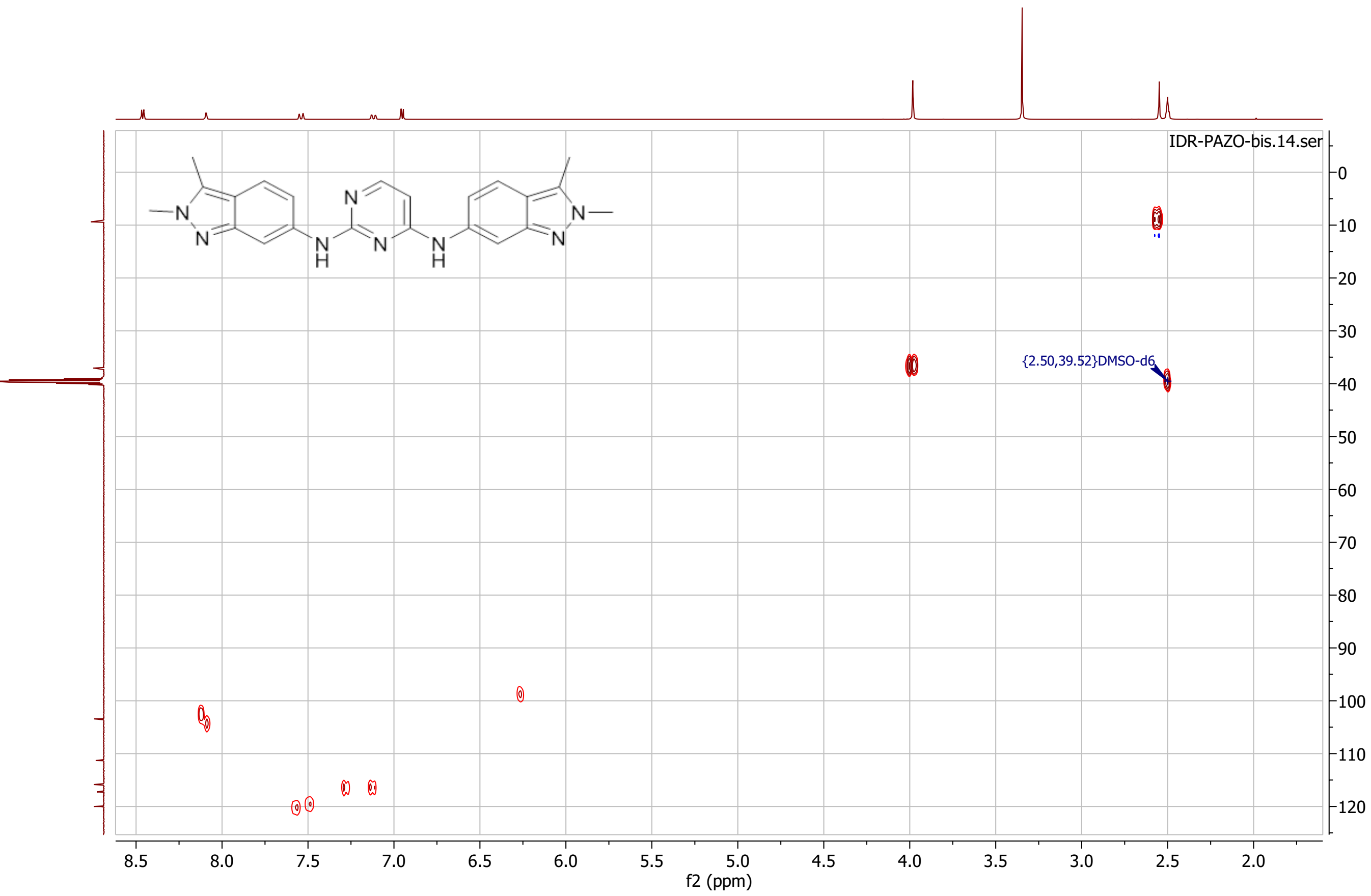

Fig.14 HSQC spectrum (400 MHz, $\left.100 \mathrm{MHz}, \mathrm{DMSO}-\mathrm{d}_{6}\right)$ of $N^{2}, N^{4}$-bis-(2,3-dimethyl-2H-indazol-6-yl)-pyrimidine-2,4-diamine (9) 


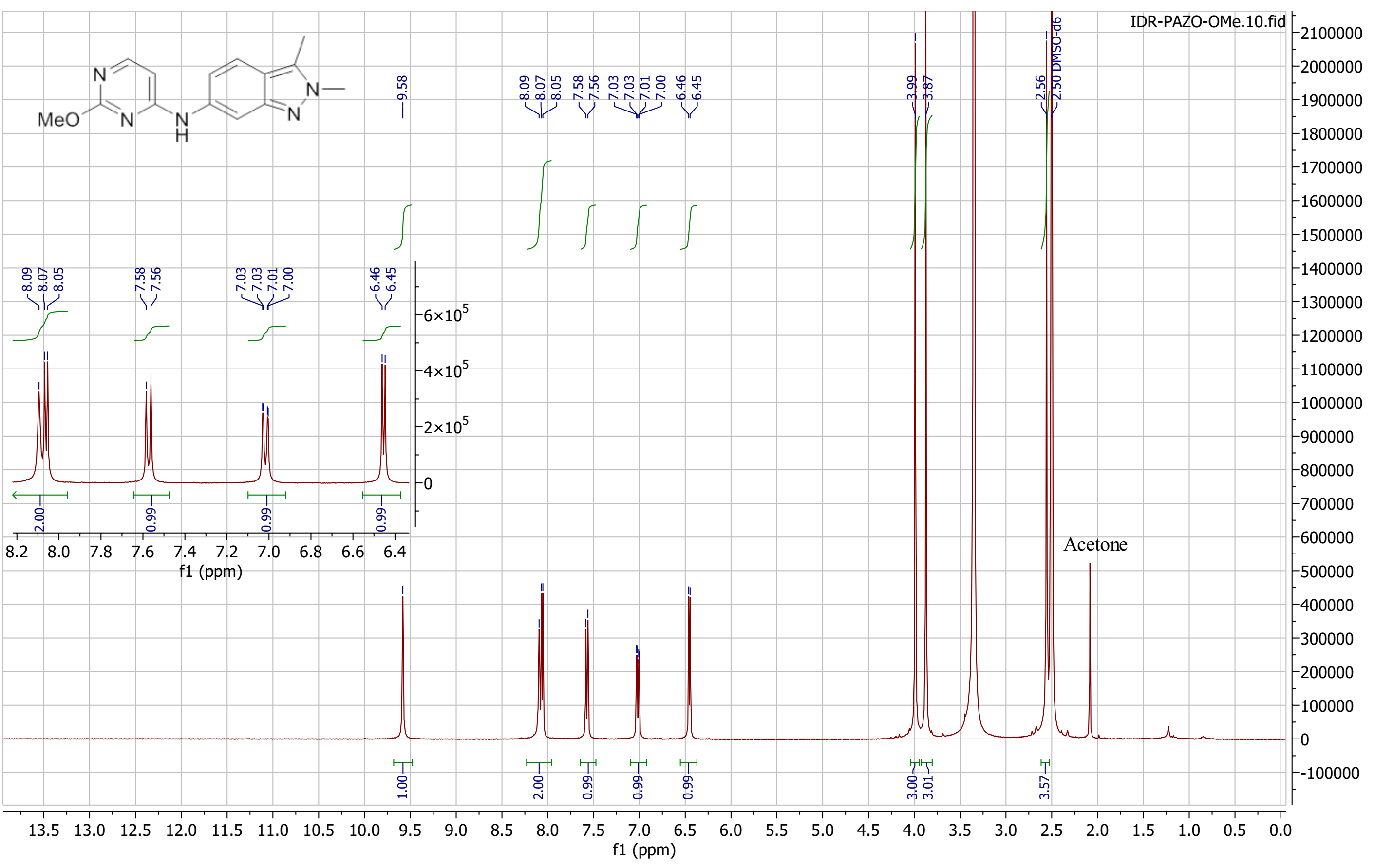

Fig. $15{ }^{1} \mathrm{H}$ NMR spectrum (400 MHz, DMSO-d 6 ) of (2,3-dimethyl-2H-indazol-6-yl)-(2-methoxypyrimidin-4-yl)-amine (10) 


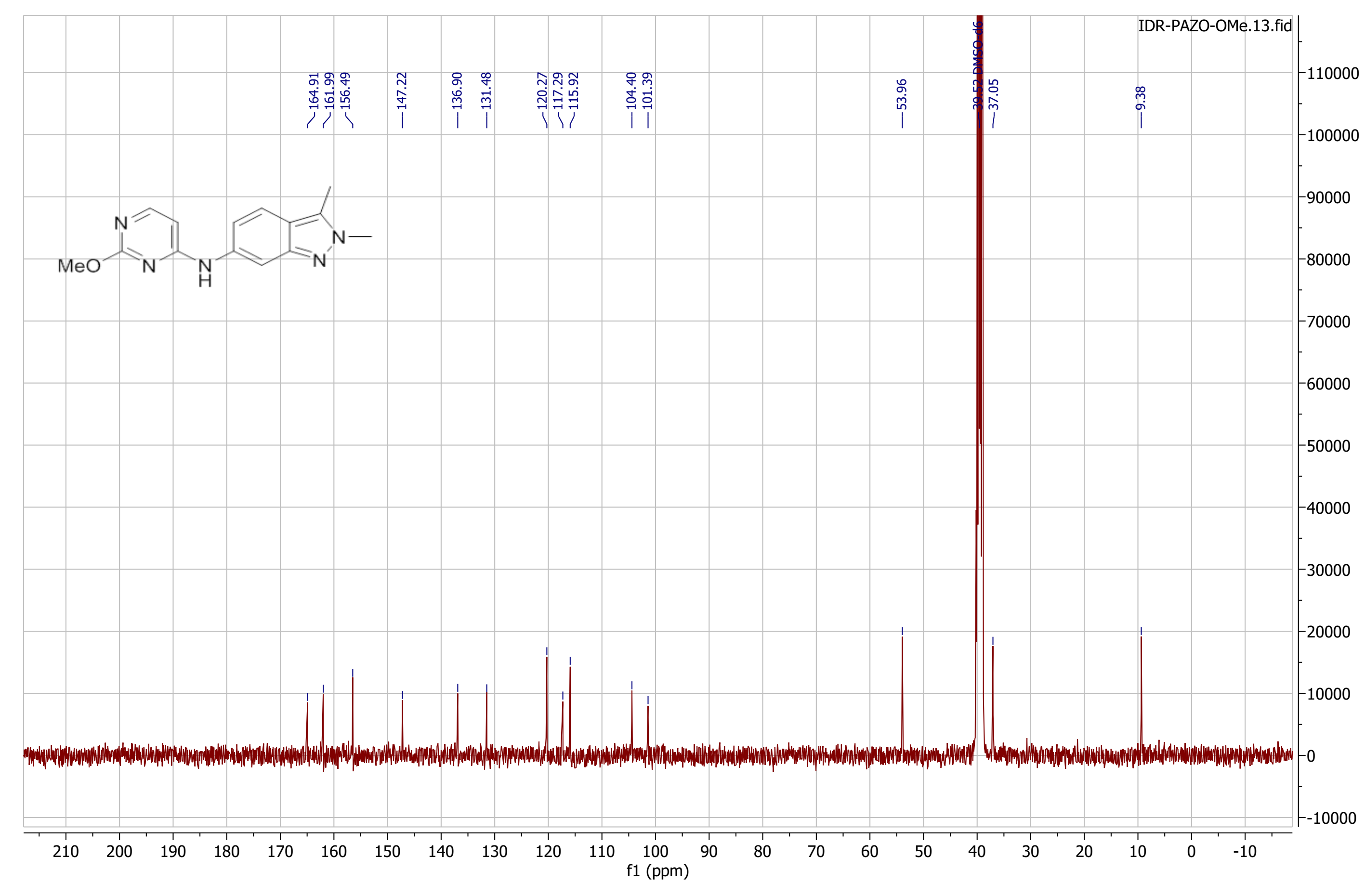

Fig. $16{ }^{13} \mathrm{C}$ NMR spectrum (100 MHz, DMSO-d 6 ) of (2,3-dimethyl-2H-indazol-6-yl)-(2-methoxypyrimidin-4-yl)-amine (10) 


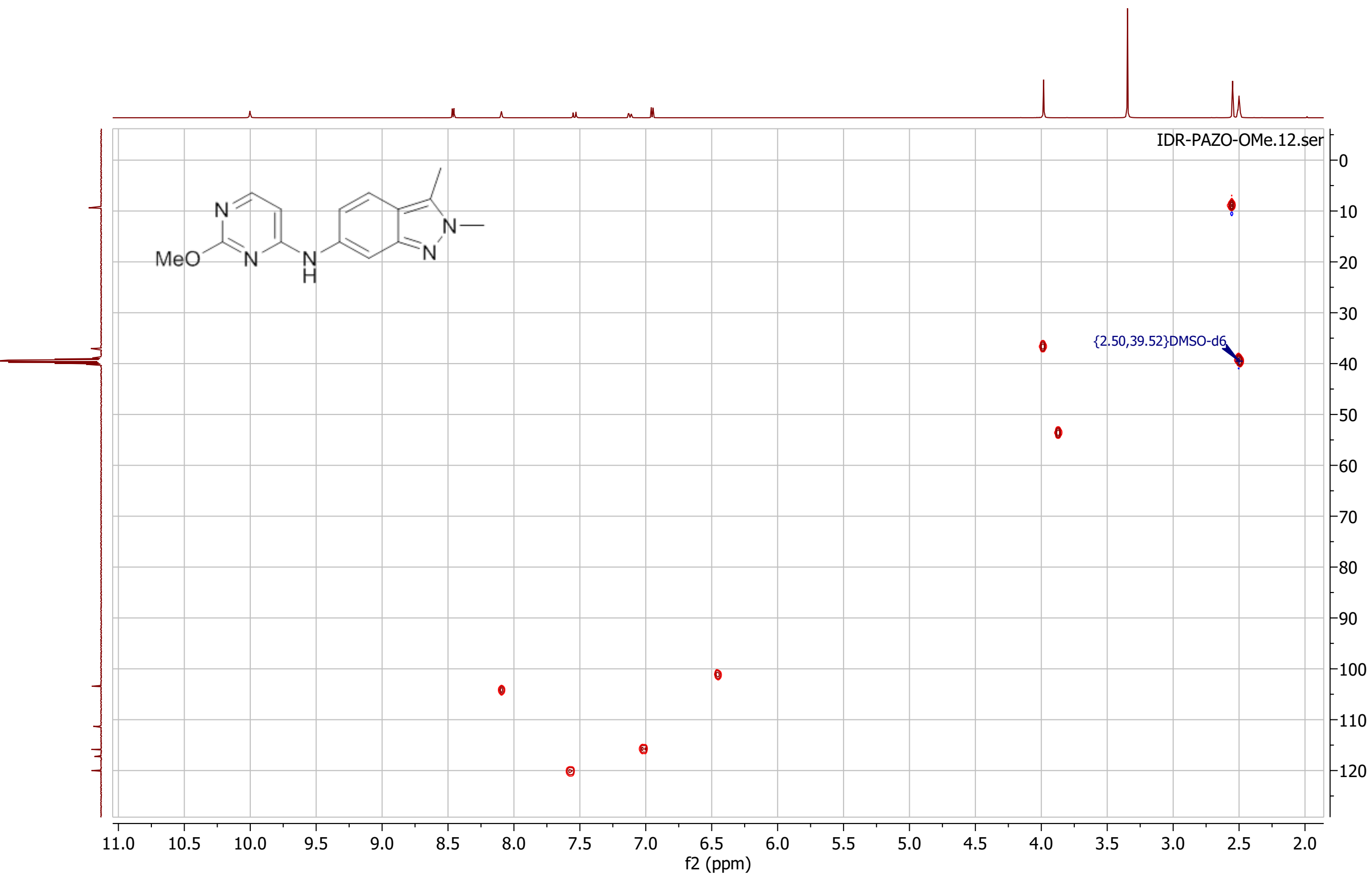

Fig. 17 HSQC spectrum (400 MHz, $\left.100 \mathrm{MHz}, \mathrm{DMSO}-\mathrm{d}_{6}\right)$ of (2,3-dimethyl-2H-indazol-6-yl)-(2-methoxypyrimidin-4-yl)-amine (10) 


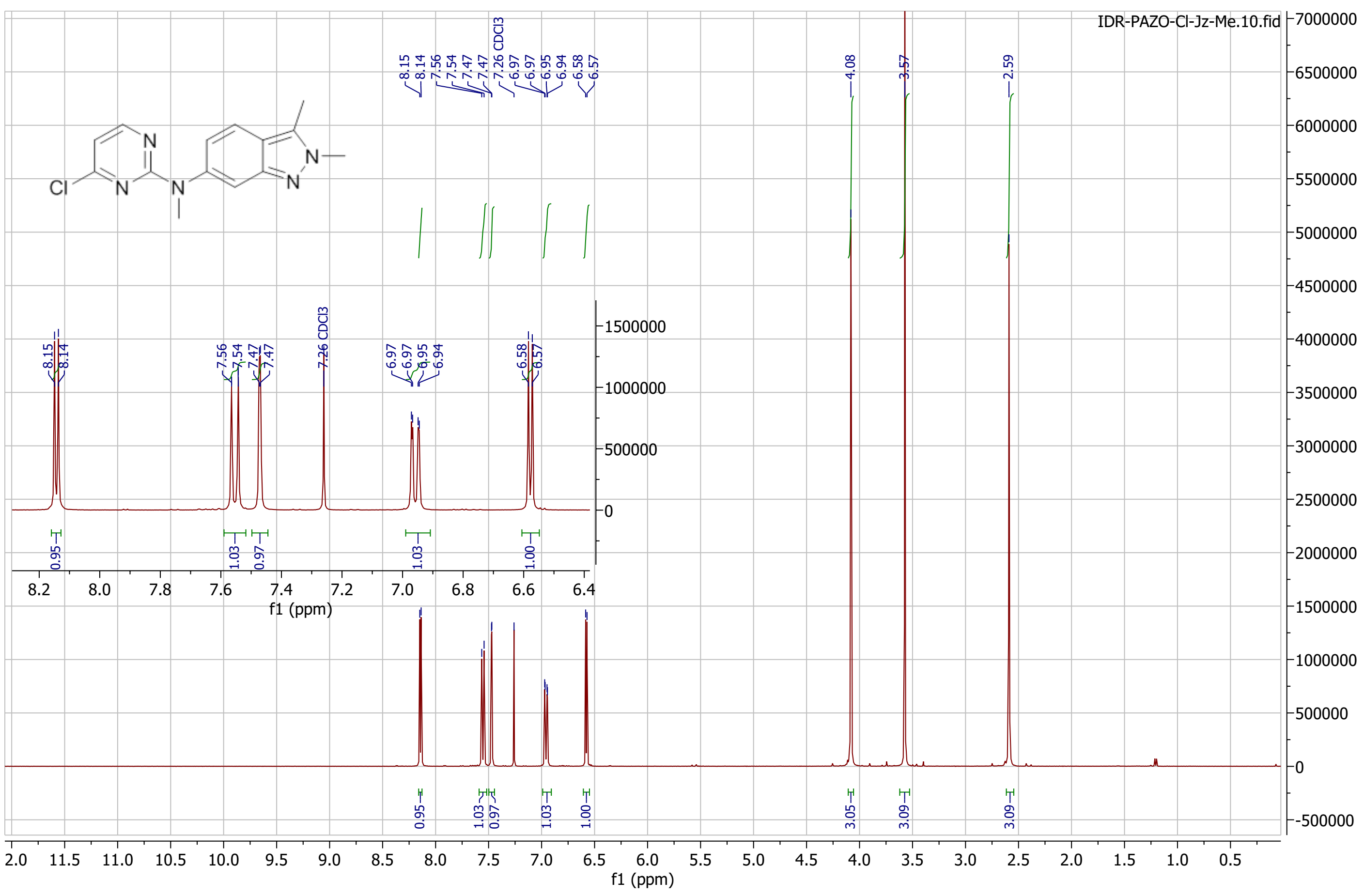

Fig. $19{ }^{1} \mathrm{H}$ NMR spectrum (400 MHz, $\mathrm{CDCl}_{3}$ ) of $N$-(4-chloropyrimidin-2-yl)- $N, 2,3$-trimethyl- $\mathrm{H}$-indazol-6-amine (12) 


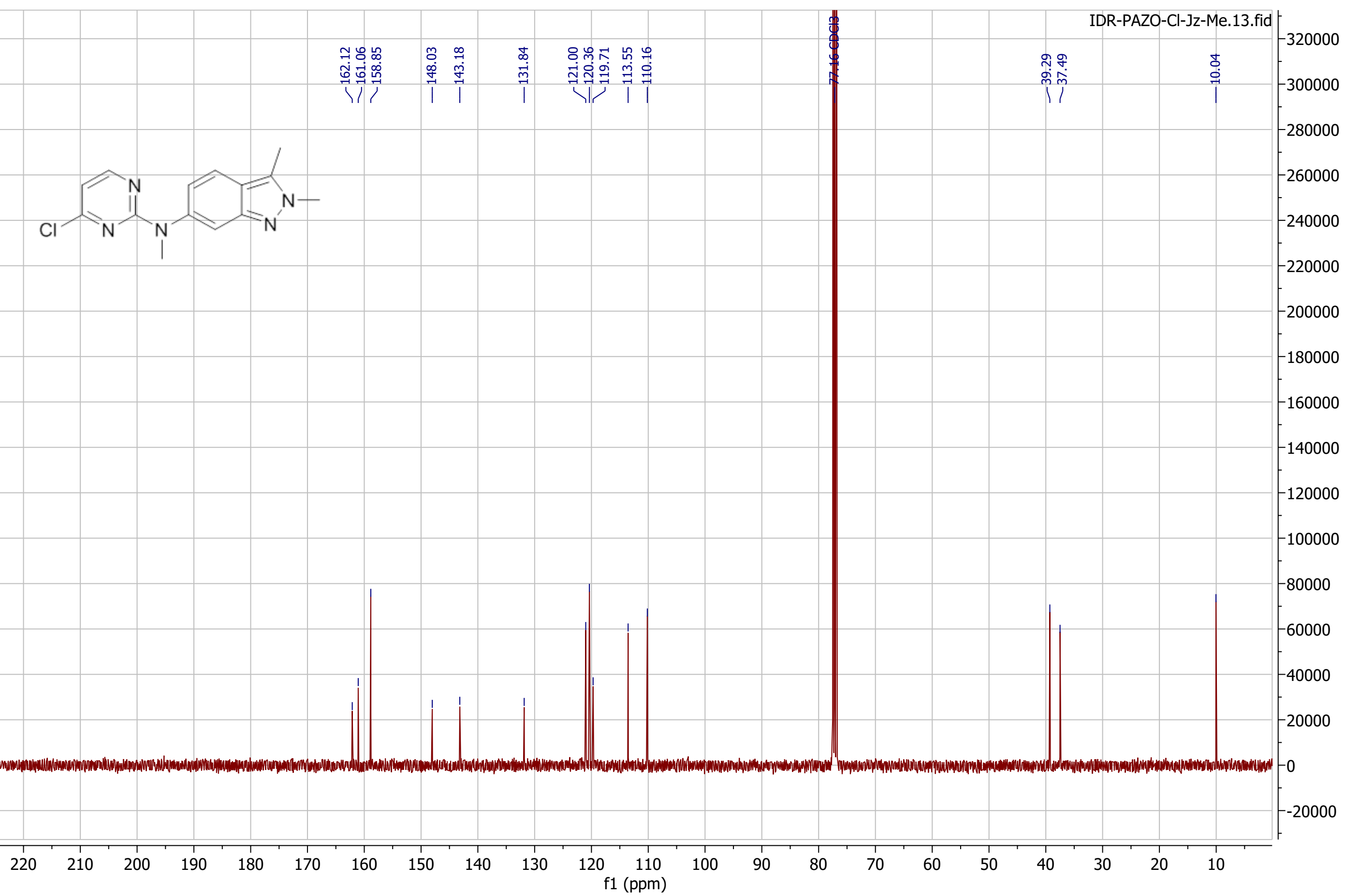

Fig. $20{ }^{13} \mathrm{C} \mathrm{NMR} \mathrm{spectrum} \mathrm{(100} \mathrm{MHz,} \mathrm{CDCl}_{3}$ ) of $N$-(4-chloropyrimidin-2-yl)- $N, 2,3$-trimethyl- $2 H$-indazol-6-amine (12) 


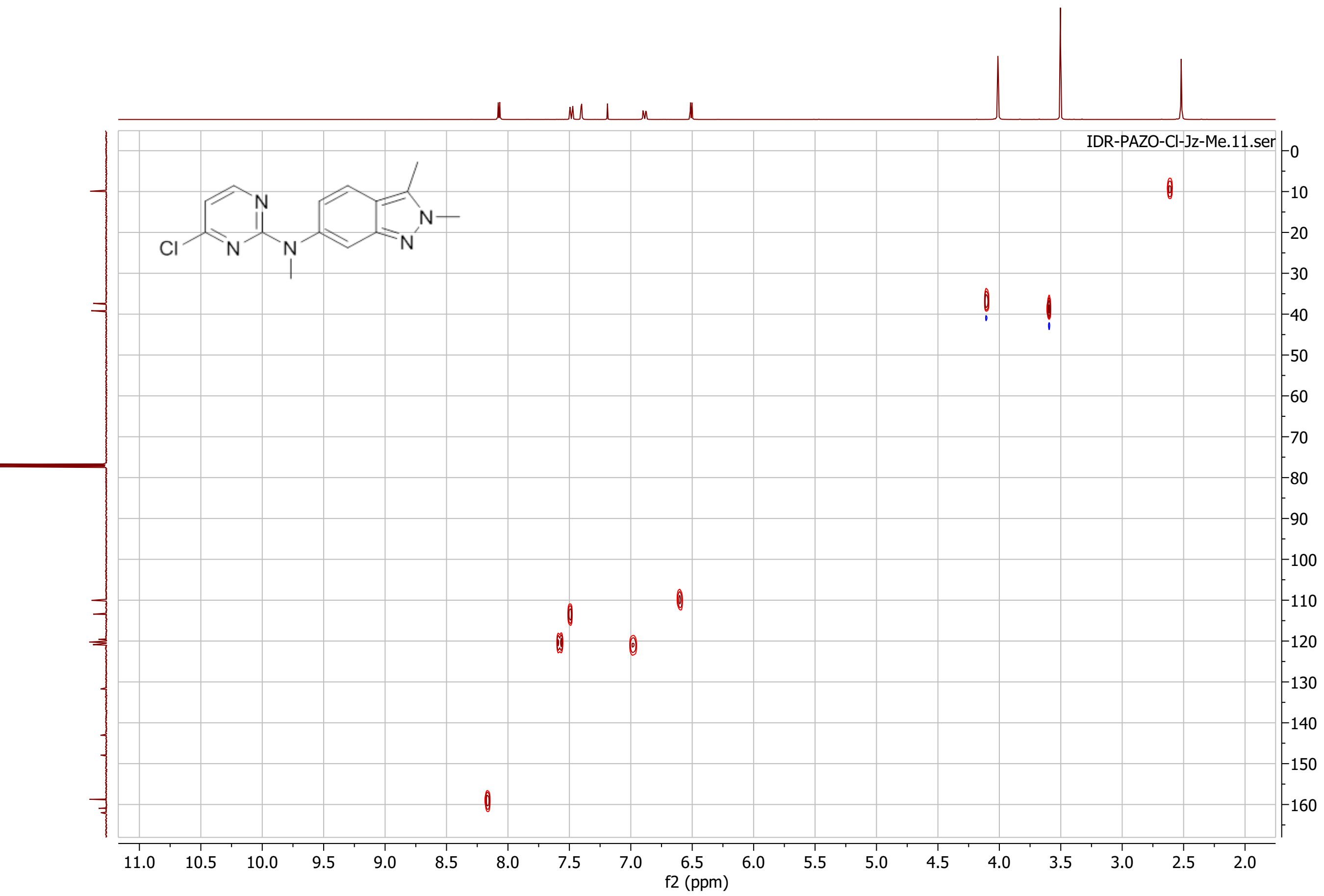

Fig.21 HSQC spectrum (400 MHz, $100 \mathrm{MHz}, \mathrm{CDCl}_{3}$ ) of $N$-(4-chloropyrimidin-2-yl)- $N, 2,3$-trimethyl-2H-indazol-6-amine (12) 


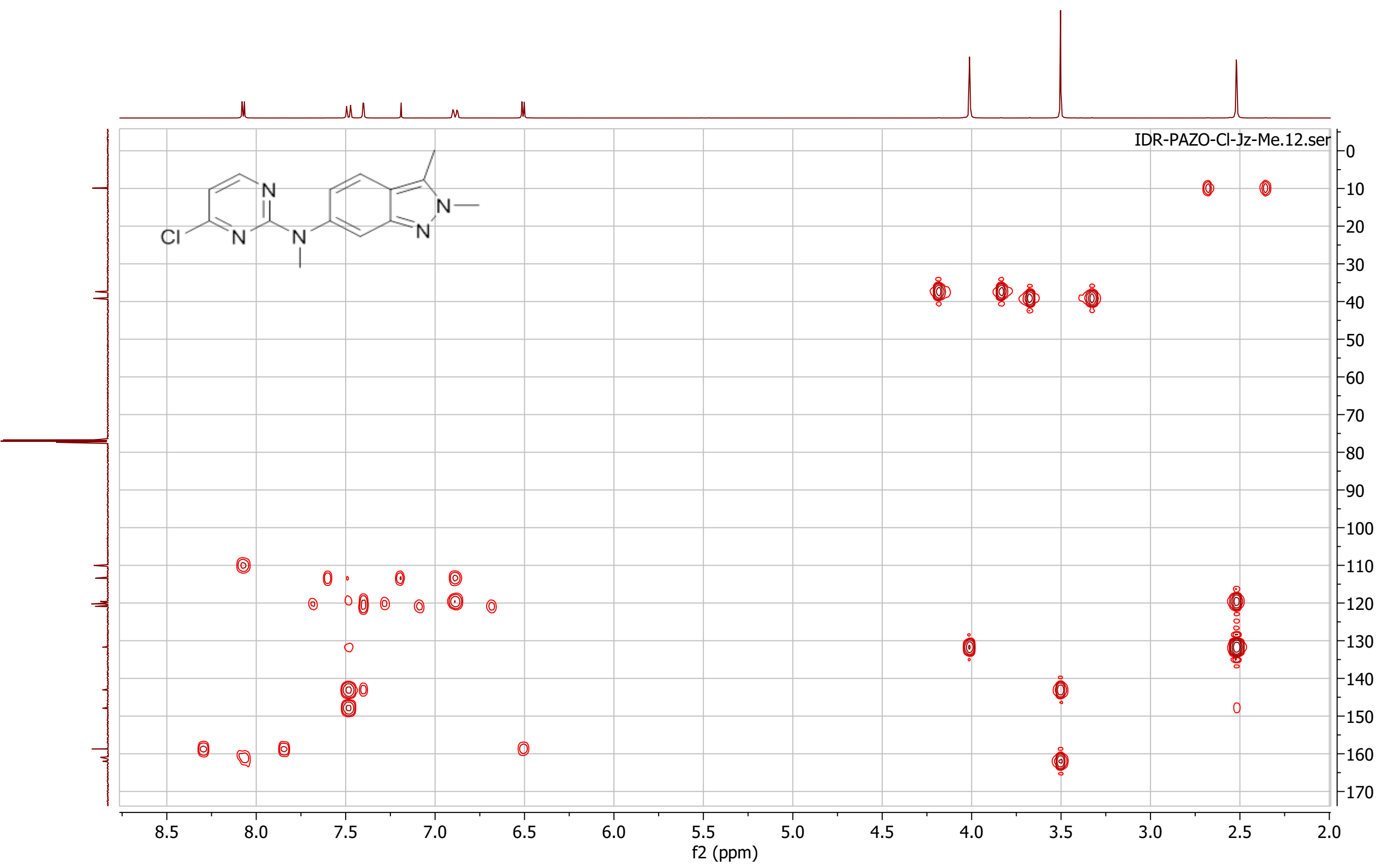

Fig.22 HMBC spectrum (400 MHz, $100 \mathrm{MHz}, \mathrm{CDCl}_{3}$ ) of $N$-(4-chloropyrimidin-2-yl)- $N, 2,3$-trimethyl-2H-indazol-6-amine (12) 


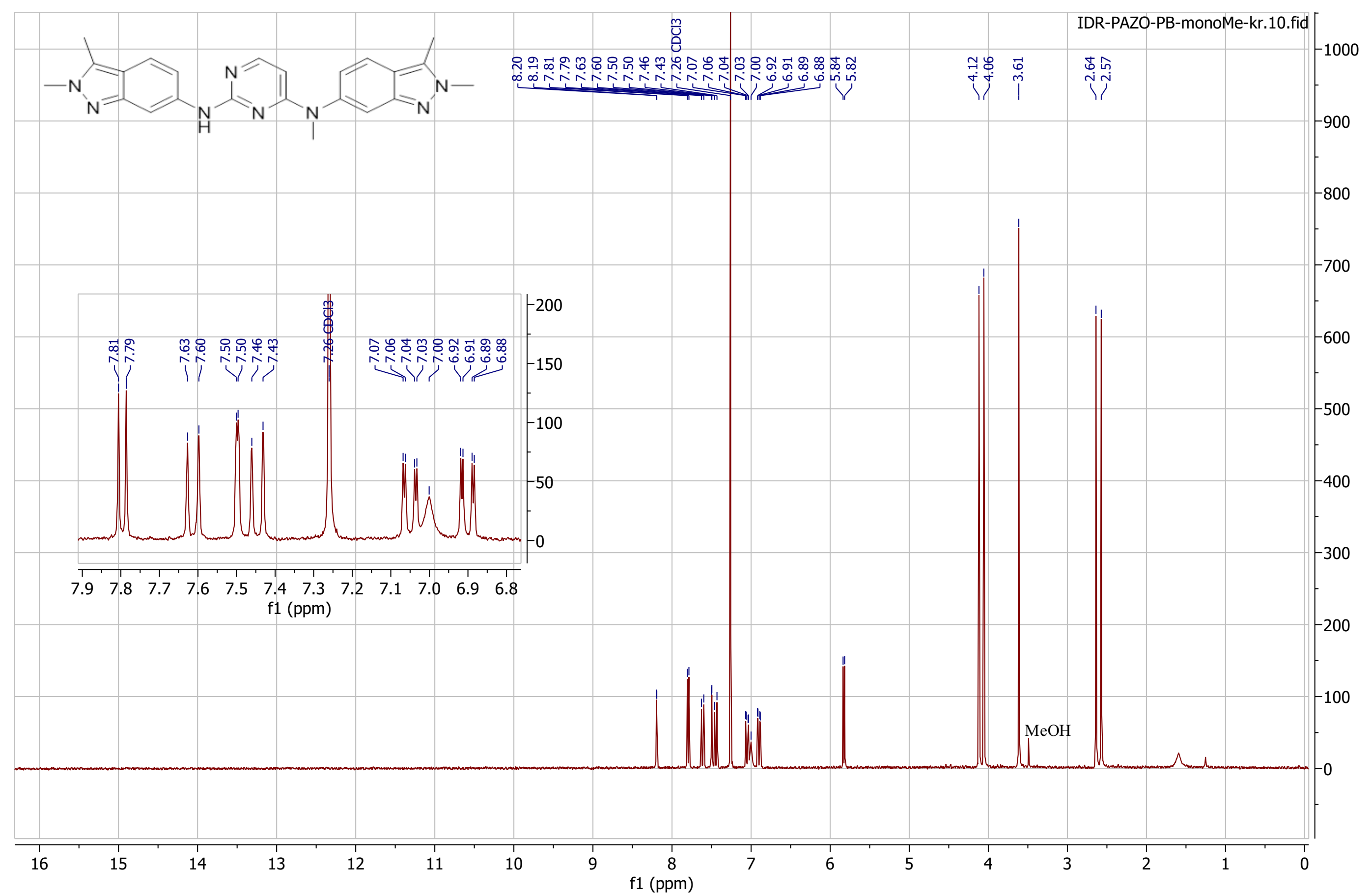

Fig. $23{ }^{1} \mathrm{H}$ NMR spectrum (400 MHz, $\mathrm{CDCl}_{3}$ ) of $N^{2}, N^{4}$-bis-(2,3-dimethyl-2H-indazol-6-yl)- $N^{4}$-methylpyrimidine-2,4-diamine (13) 


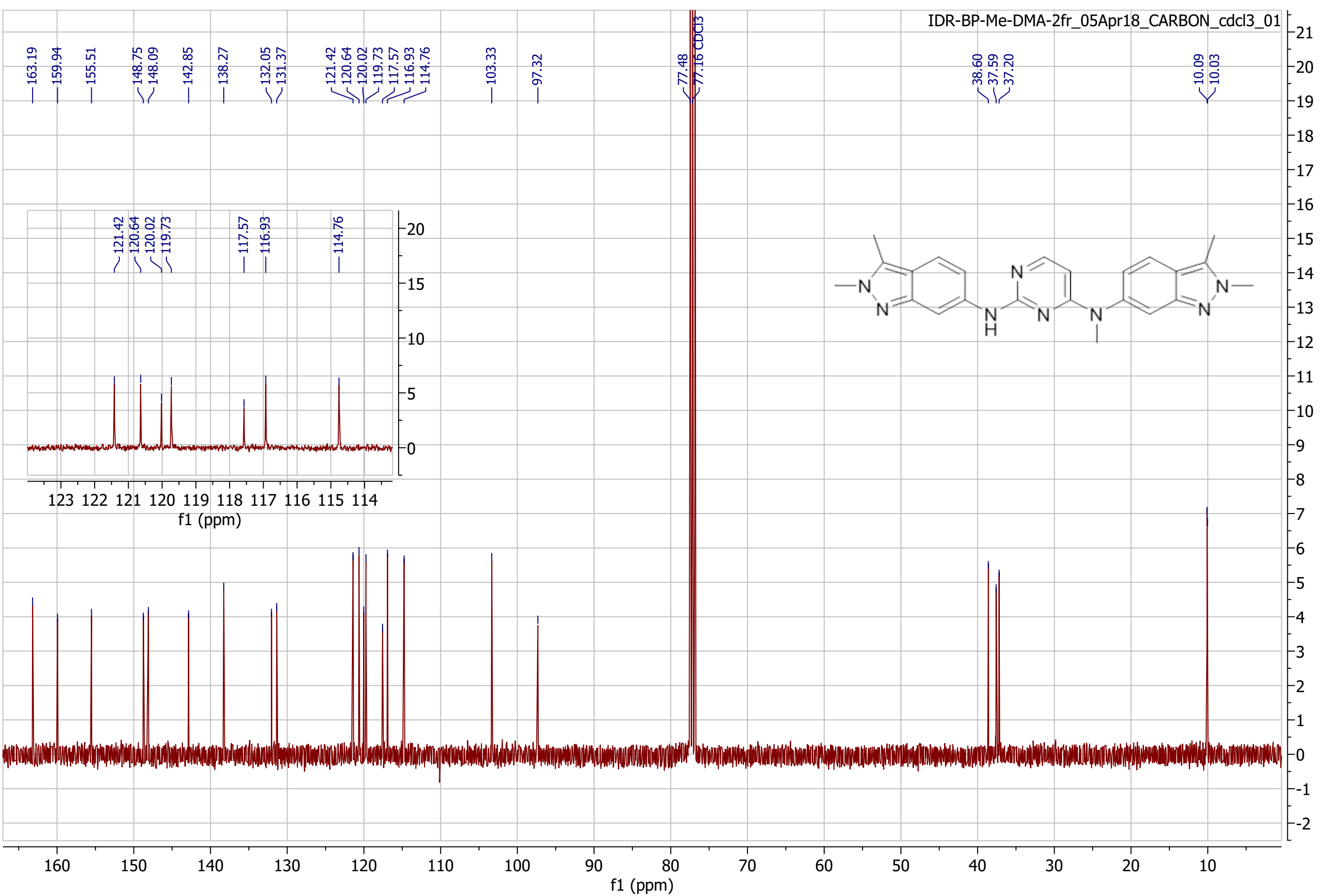

Fig. $24{ }^{13} \mathrm{C}$ NMR spectrum (100 MHz, $\mathrm{CDCl}_{3}$ ) of $N^{2}, N^{4}$-bis-(2,3-dimethyl- $2 H$-indazol-6-yl)- $N^{4-}$ methylpyrimidine-2,4-diamine (13) 


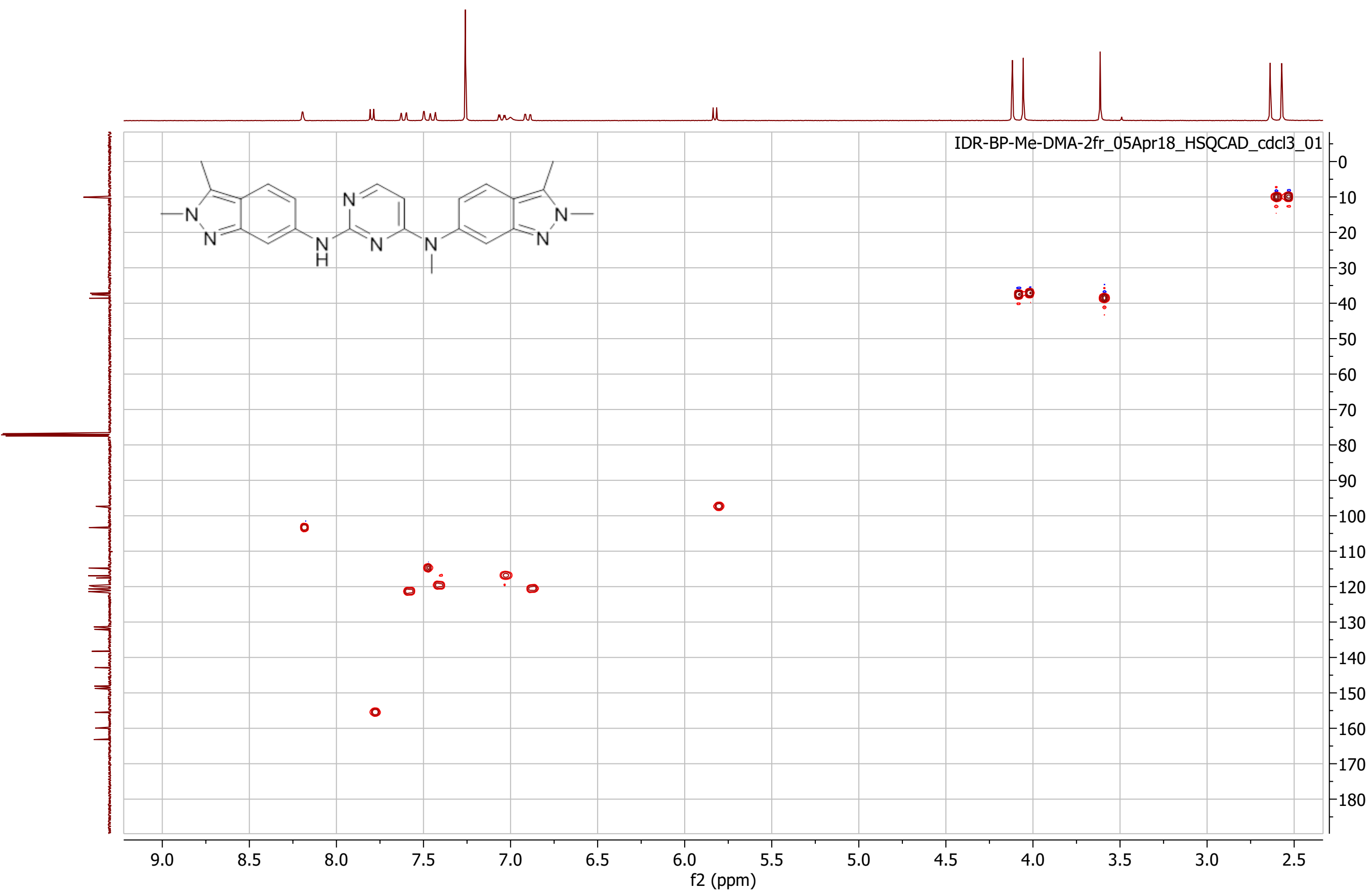

Fig.25 HSQC spectrum (400 MHz, $100 \mathrm{MHz}, \mathrm{CDCl}_{3}$ ) of $N^{2}, N^{4}$-bis-(2,3-dimethyl-2H-indazol-6-yl)- $N^{4-}$ methylpyrimidine-2,4-diamine (13) 


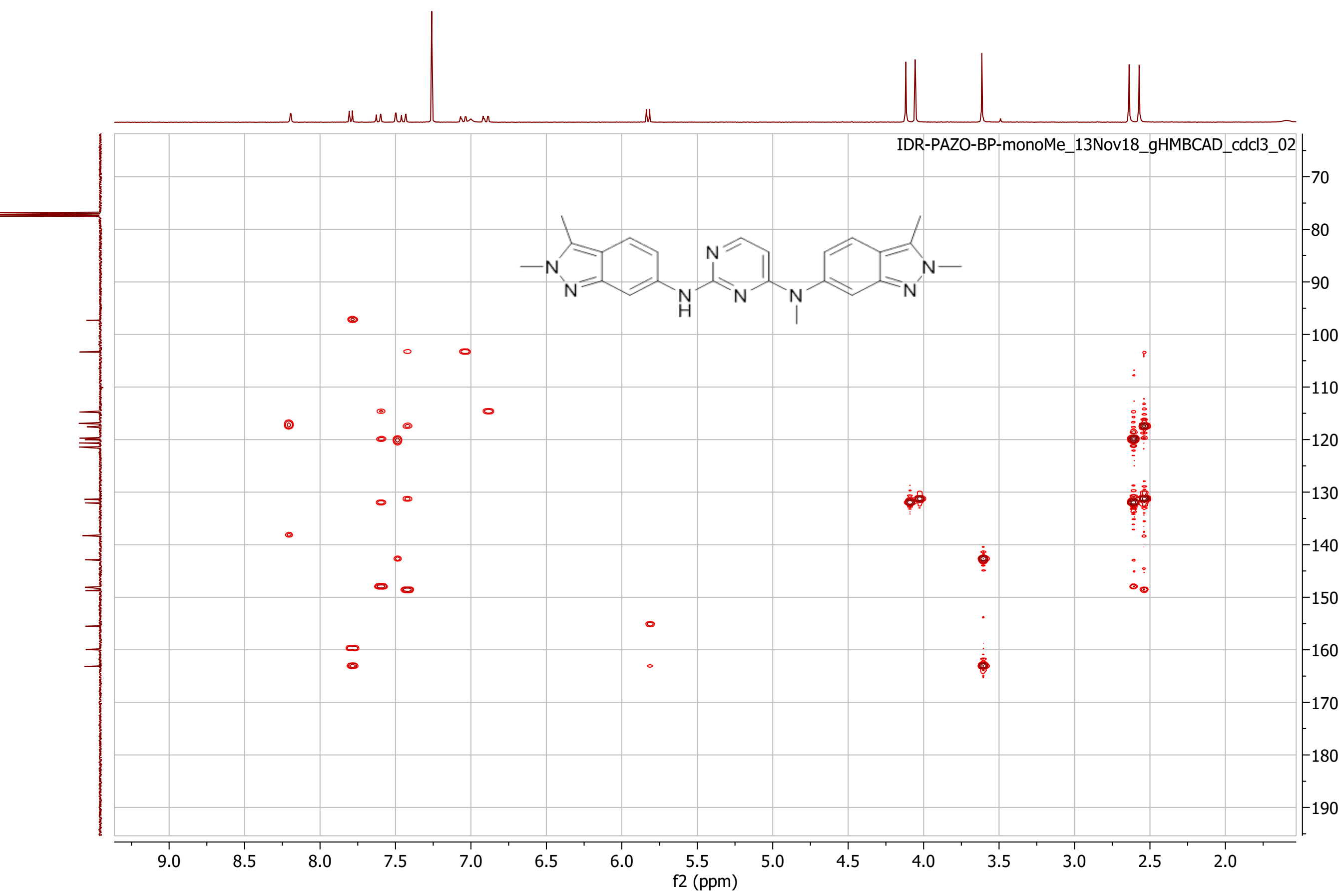

Fig.26 HMBC spectrum (400 MHz, $\left.100 \mathrm{MHz}, \mathrm{CDCl}_{3}\right)$ of $N^{2}, N^{4}$-bis-(2,3-dimethyl-2H-indazol-6-yl)- $N^{4}$-methylpyrimidine-2,4-diamine (13) 


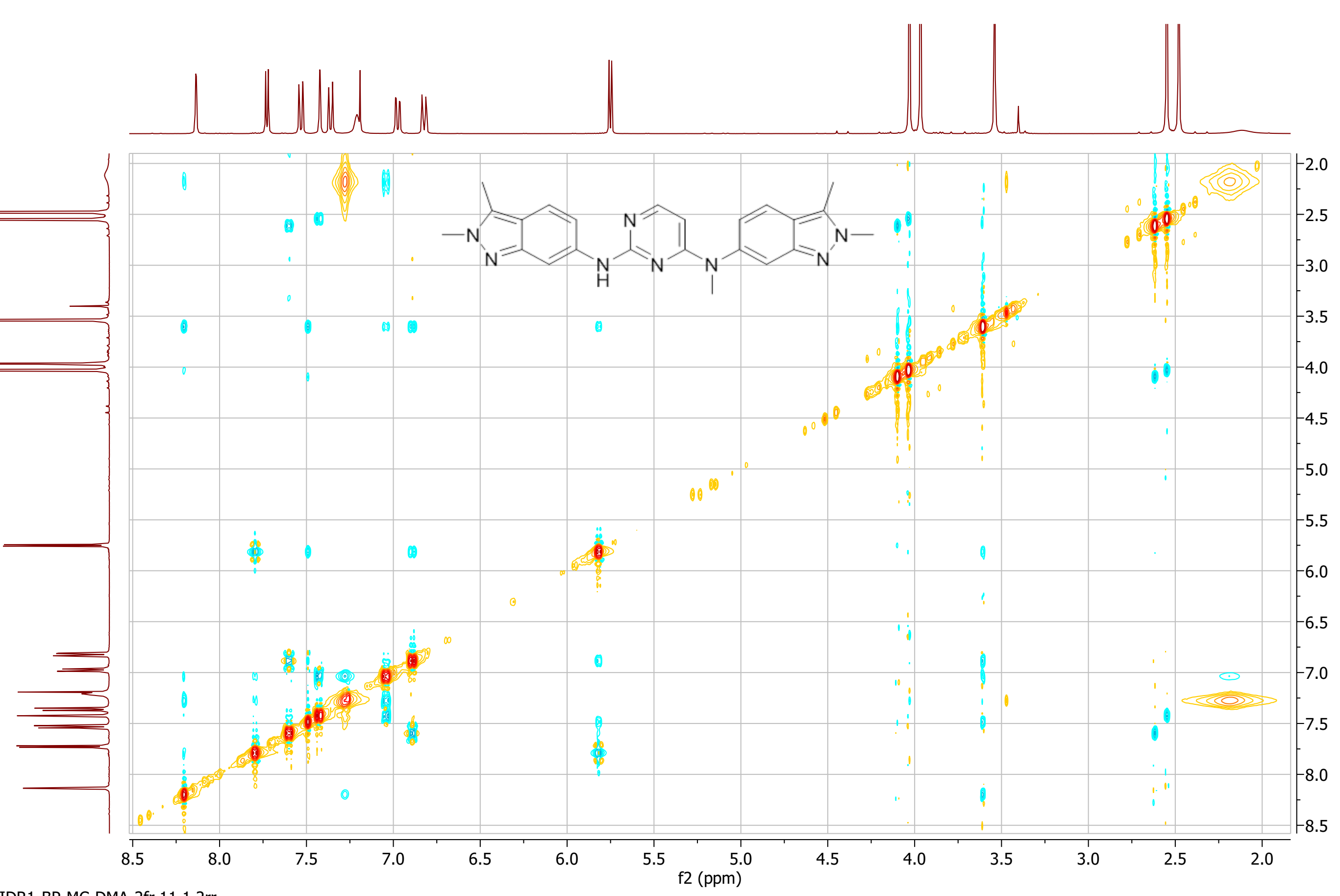

IDR1-BP-MC-DMA-2fr.11.1.2rr -

Fig.27 NOESY spectrum (400 MHz, $\left.\mathrm{CDCl}_{3}\right)$ of $N^{2}, N^{4}$-bis-(2,3-dimethyl-2H-indazol-6-yl)- $N^{4}$-methylpyrimidine-2,4-diamine (13) 


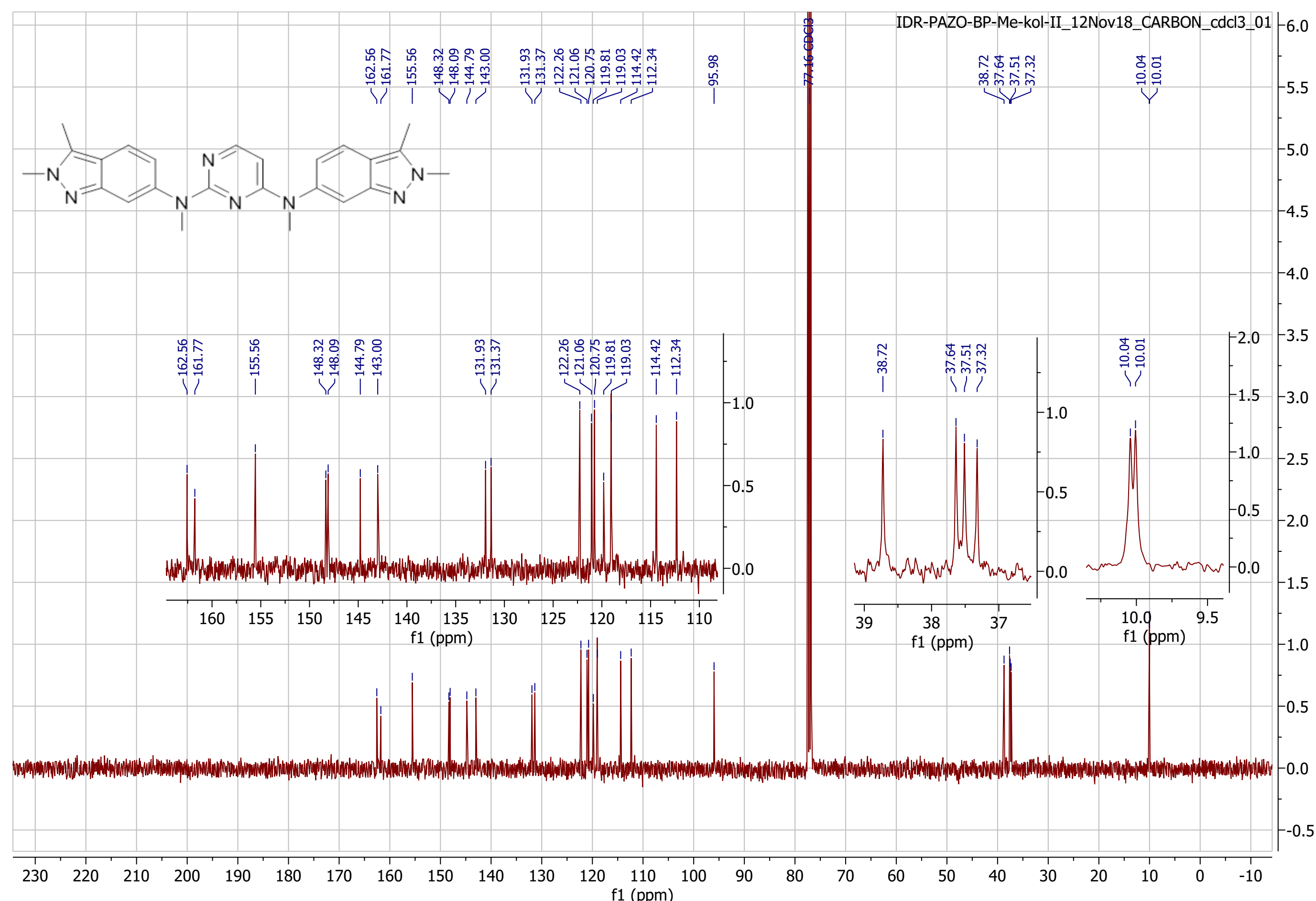

Fig. $28{ }^{13} \mathrm{C}-\mathrm{NMR}$ spectrum $\left(100 \mathrm{MHz}, \mathrm{CDCl}_{3}\right)$ of $N^{2}, N^{4}$-bis-(2,3-dimethyl-2 $H$-indazol-6-yl)- $N^{2}, N^{4}$-dimethylpyrimidine-2,4-diamine (14) 


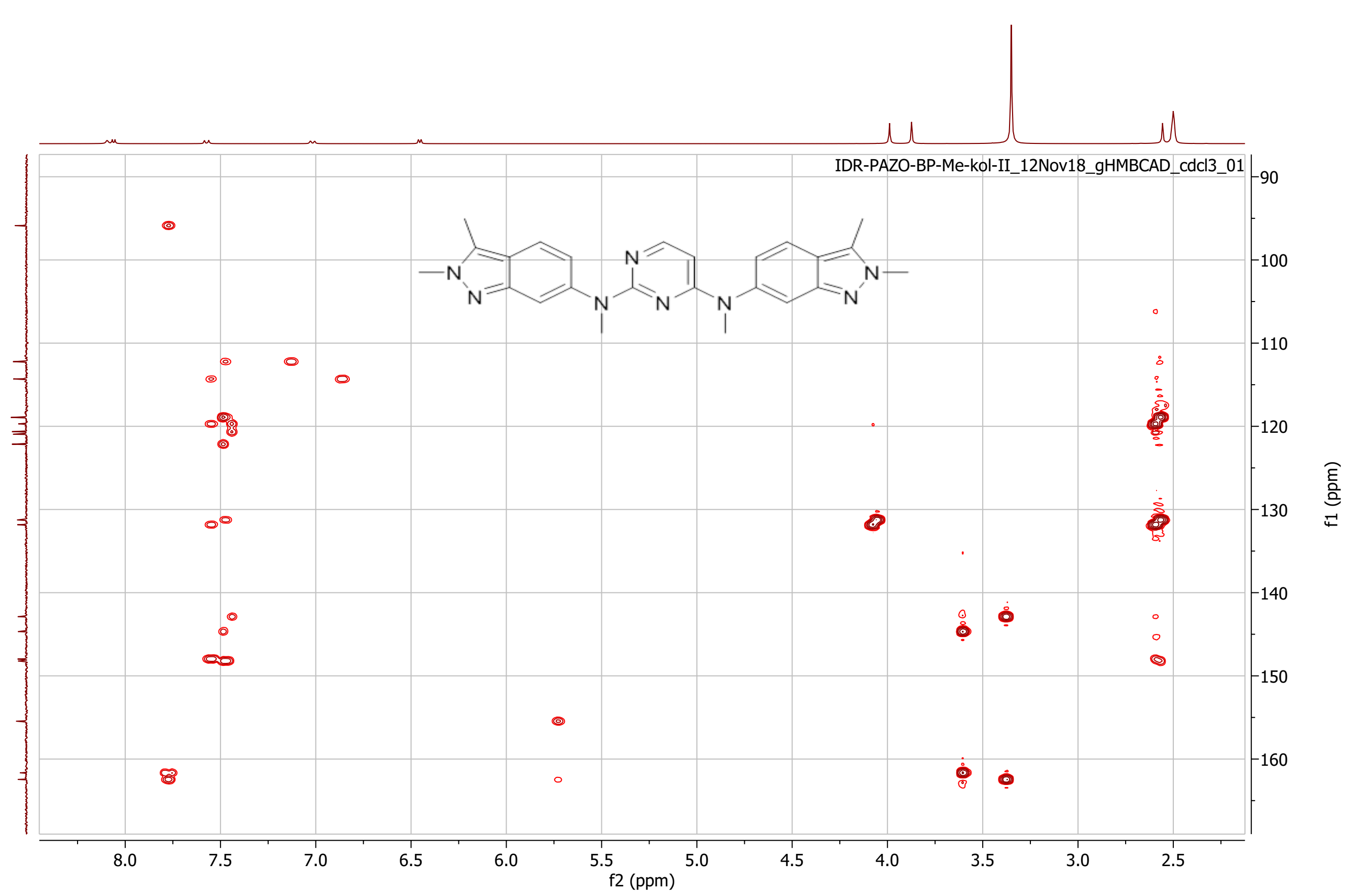

Fig. $29 \mathrm{HMBC}$ spectrum (400 MHz, 100MHz, $\mathrm{CDCl}_{3}$ ) of $N^{2}, N^{4}$-bis-(2,3-dimethyl-2H-indazol-6-yl)- $N^{2}, N^{4}$-dimethylpyrimidine-2,4-diamine (14) 
U

M

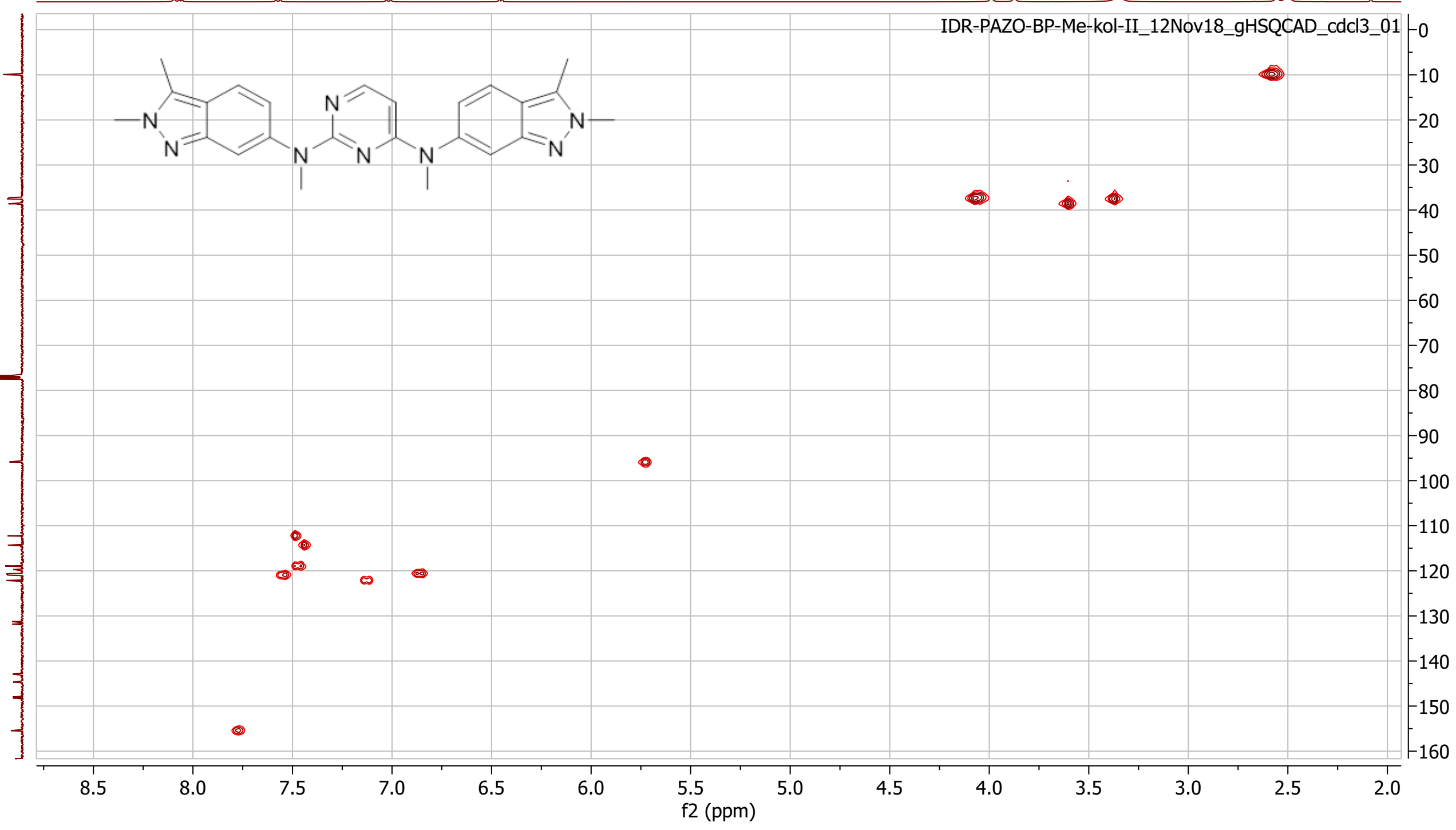

Fig.30 HSQC spectrum (400 MHz, $\left.100 \mathrm{MHz}, \mathrm{CDCl}_{3}\right)$ of $N^{2}, N^{4}$-bis-(2,3-dimethyl-2H-indazol-6-yl)- $N^{2}, N^{4}$-dimethylpyrimidine-2,4-diamine (14) 


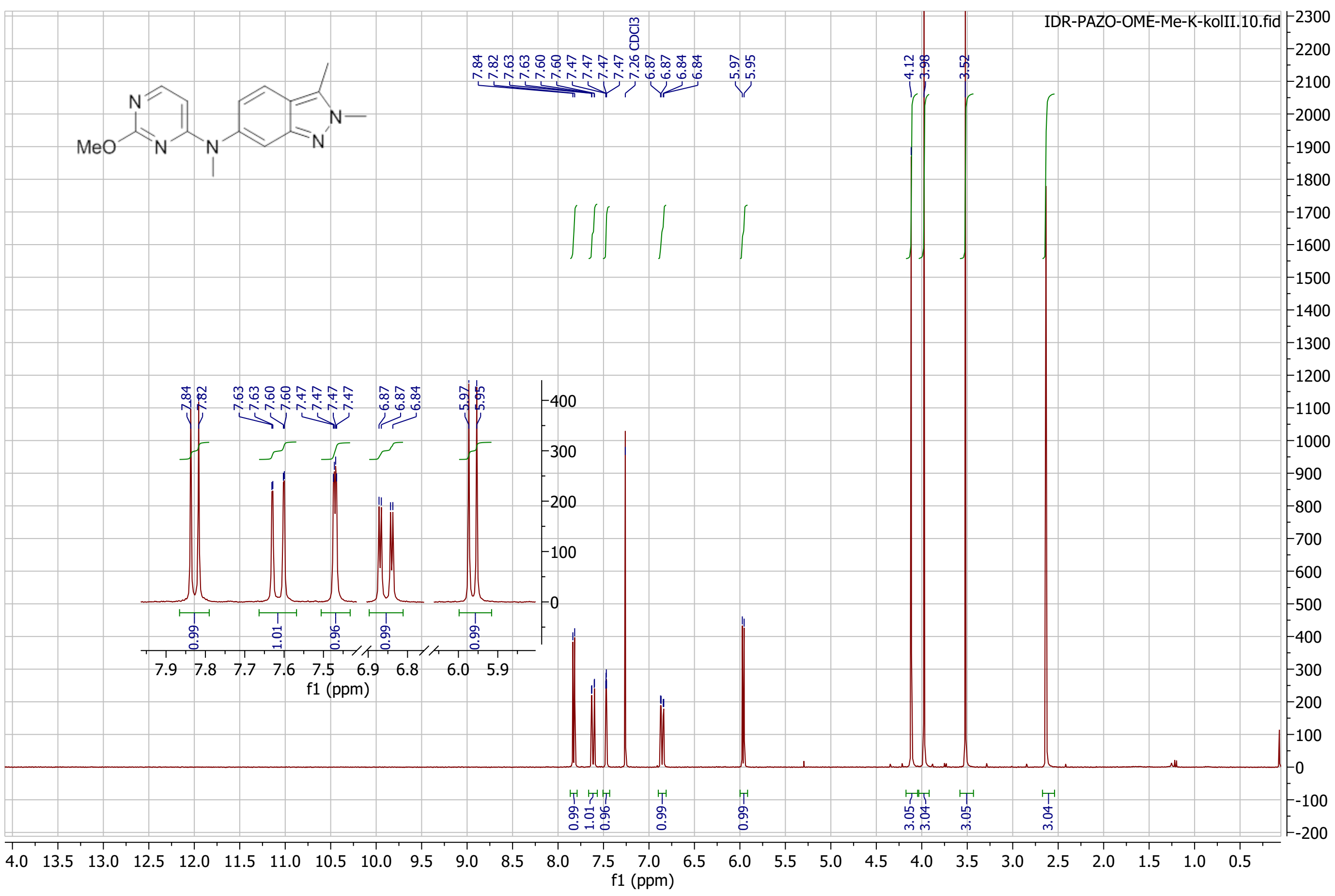

Fig. $31{ }^{1} \mathrm{H}$ NMR spectrum (300 MHz, $\mathrm{CDCl}_{3}$ ) of (2,3-dimethyl-2H-indazol-6-yl)-(2-methoxypyrimidin-4-yl)methylamine (15) 


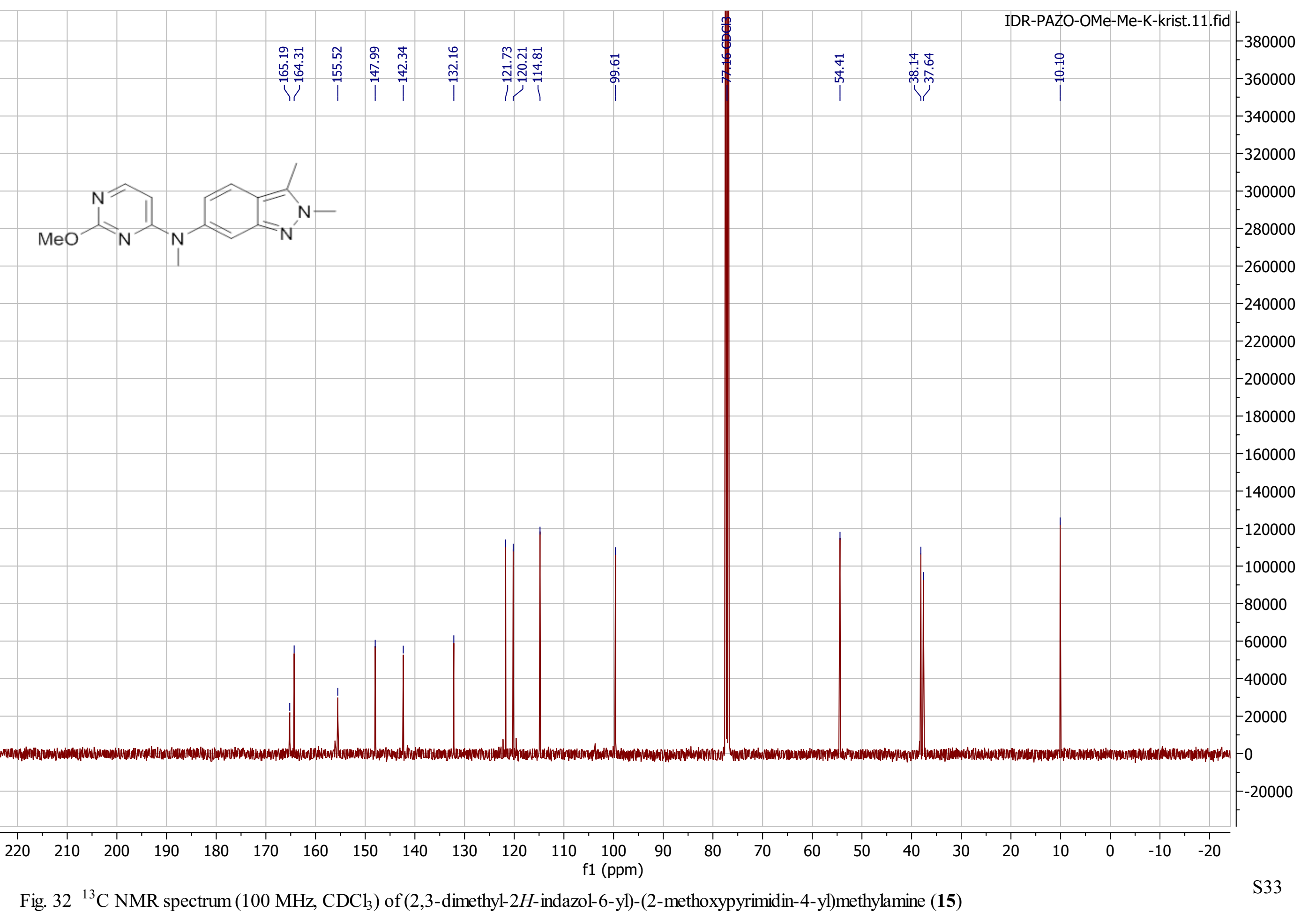




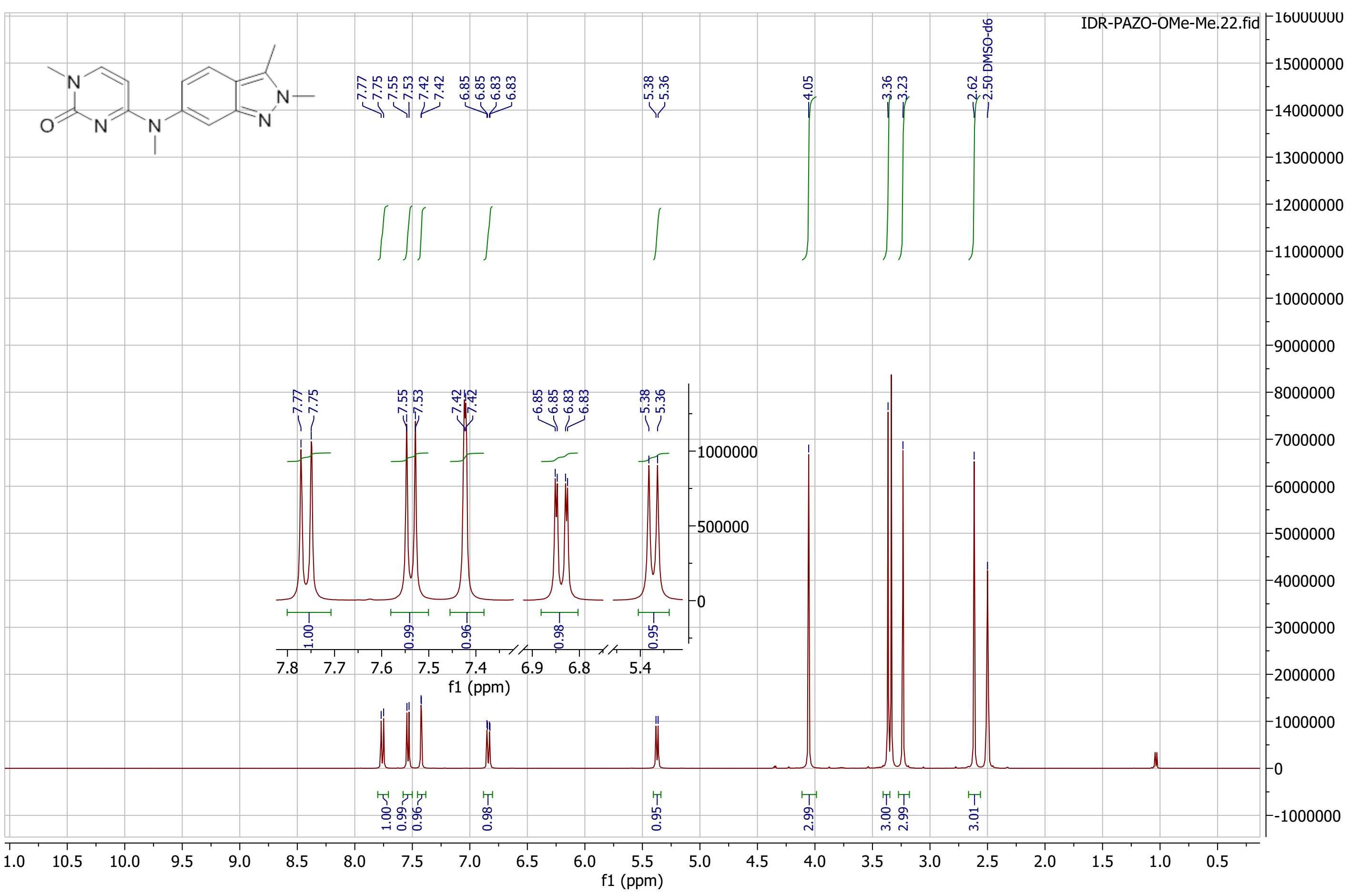

Fig. $33{ }^{1} \mathrm{H}-\mathrm{NMR}$ spectrum (300MHz, DMSO-d $\left.{ }_{6}\right)$ of 4-[(2,3-dimethyl-2H-indazol-6-yl)methylamino]-1-metil-1H-pirimidin-2-one (16) 


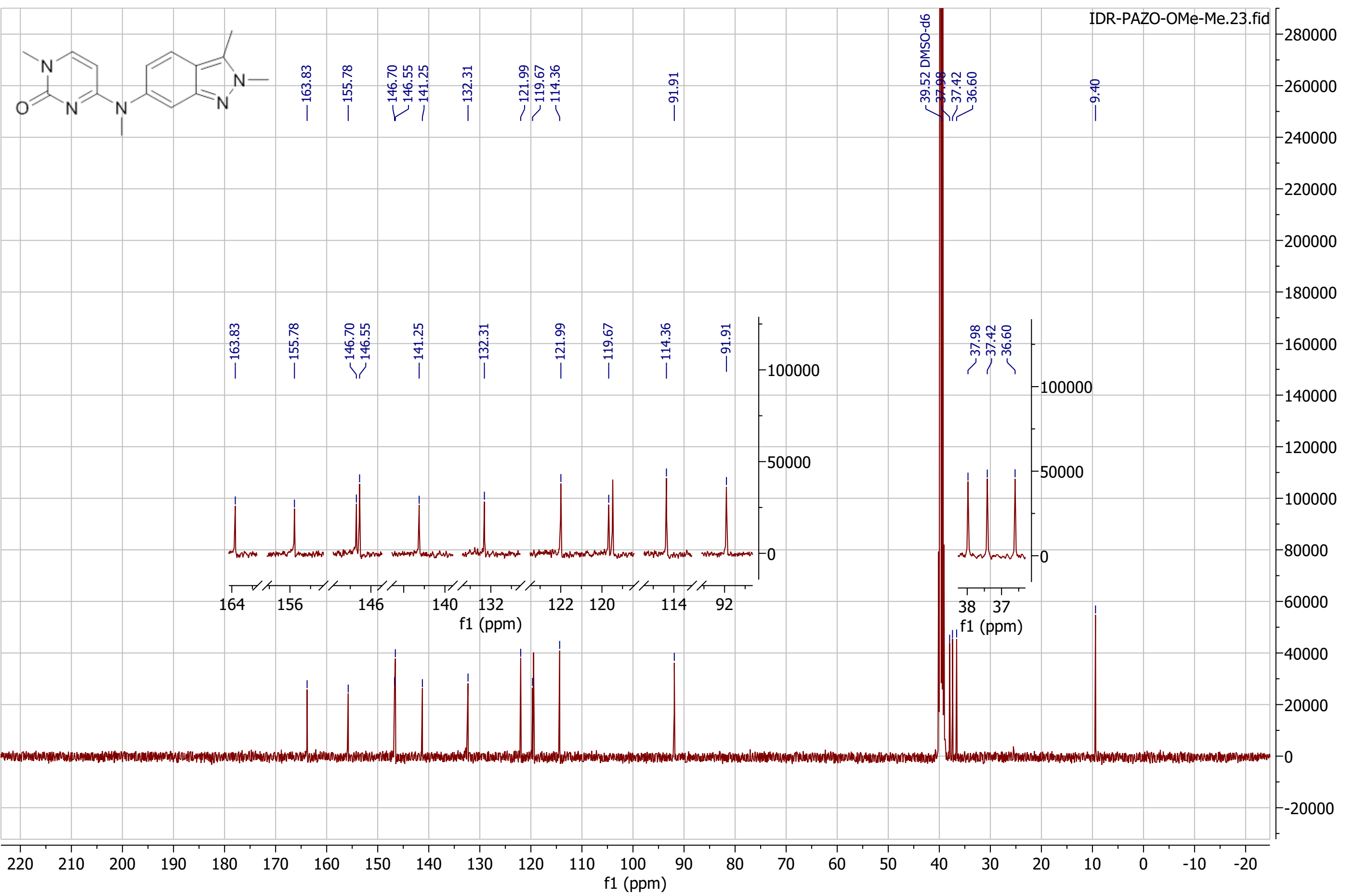

Fig. $34{ }^{13} \mathrm{C}-\mathrm{NMR}$ spectrum $\left(100 \mathrm{MHz}, \mathrm{DMSO}-\mathrm{d}_{6}\right)$ of 4-[(2,3-dimethyl-2H-indazol-6-yl)methylamino]-1-metil-1H-pirimidin-2-one (16) 


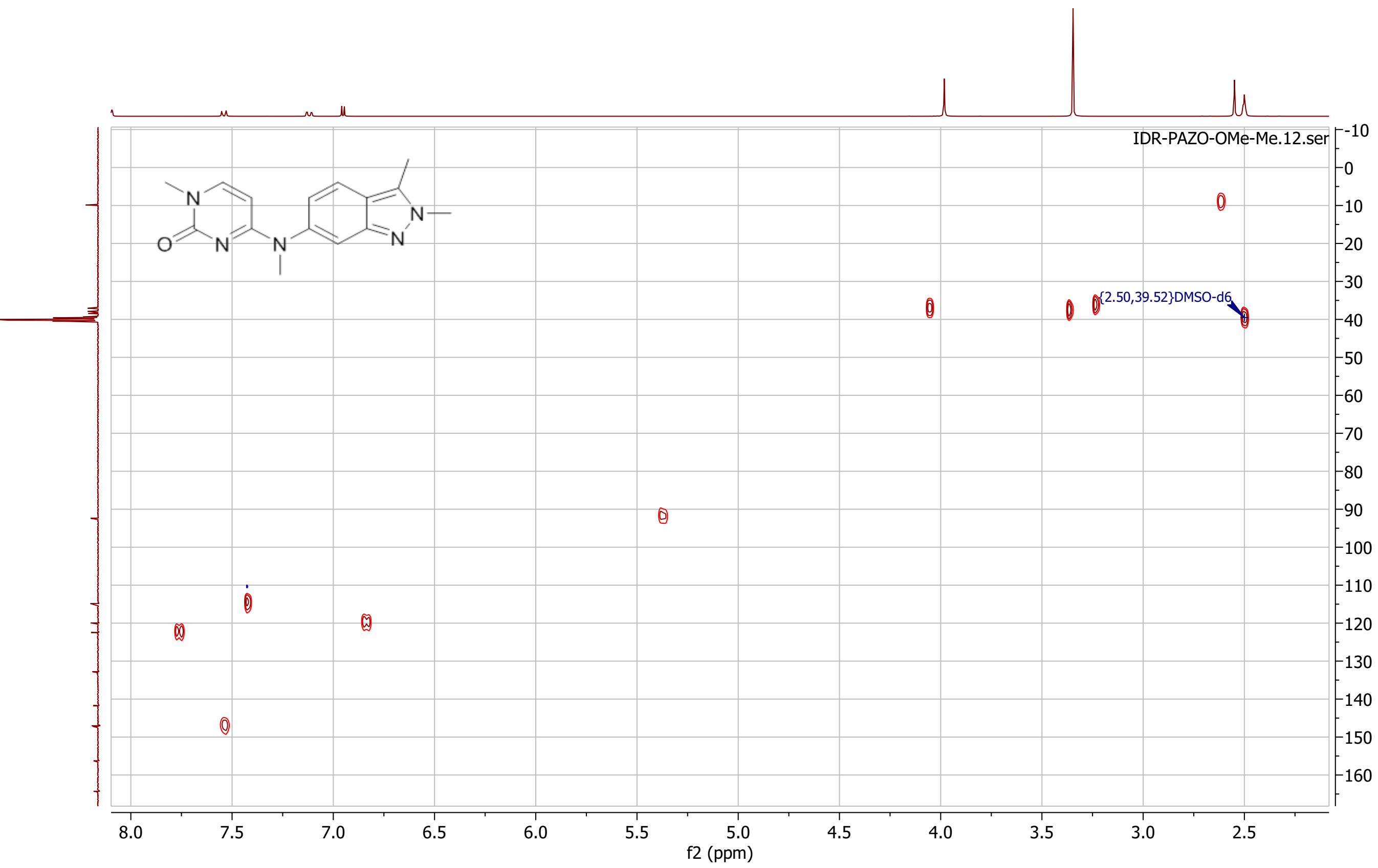

Fig. 35 HSQC spectrum (400 MHz, $\left.100 \mathrm{MHz}, \mathrm{DMSO}-\mathrm{d}_{6}\right)$ of 4-[(2,3-dimethyl-2H-indazol-6-yl)methylamino]-1-metil-1H-pirimidin-2-one (16) 


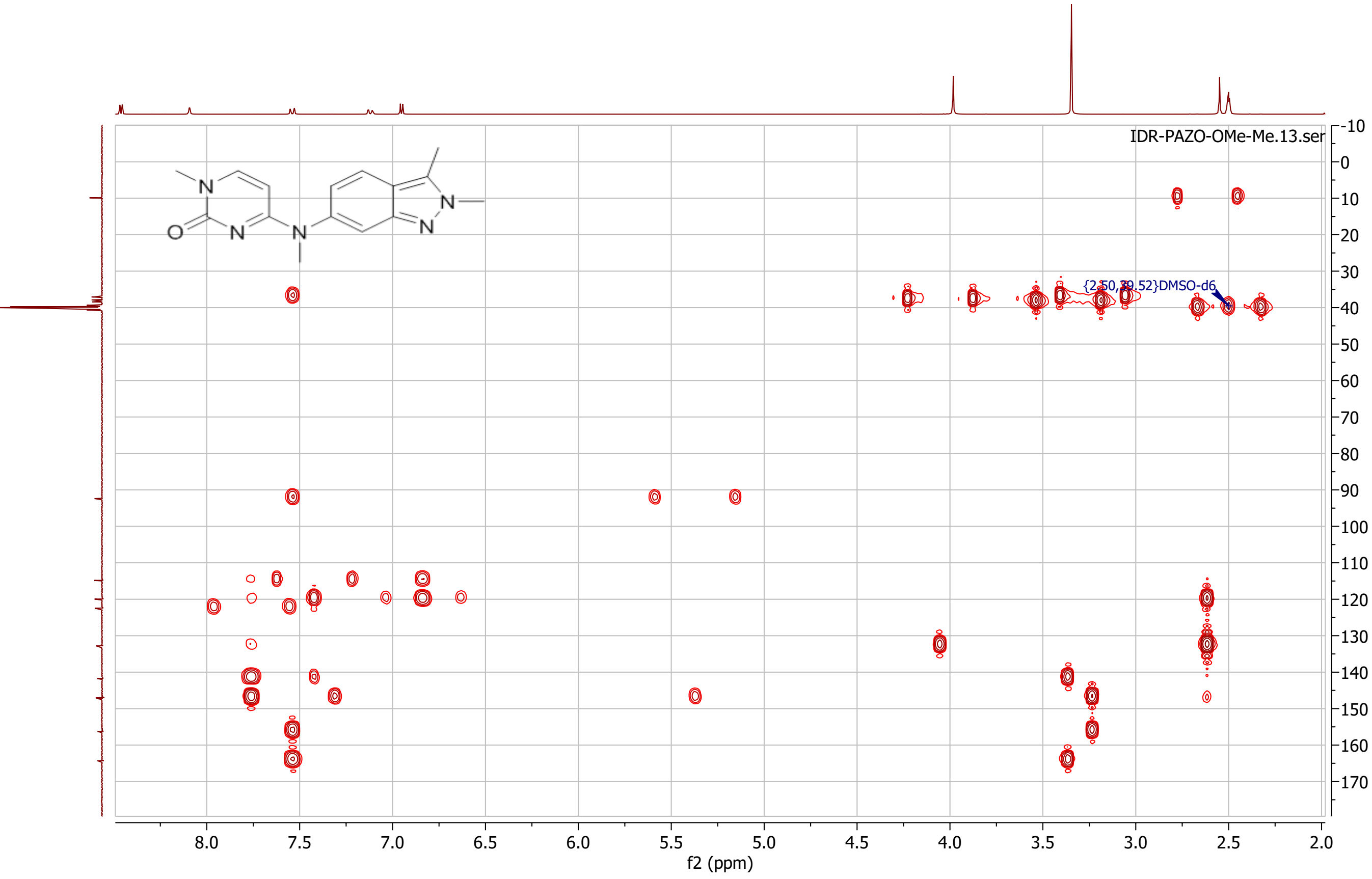

Fig. $36 \mathrm{HMBC}$ spectrum (400 MHz, 100MHz, DMSO-d $\left.{ }_{6}\right)$ of 4-[(2,3-dimethyl-2H-indazol-6-yl)methylamino]-1-metil-1H-pirimidin-2-one (16) 


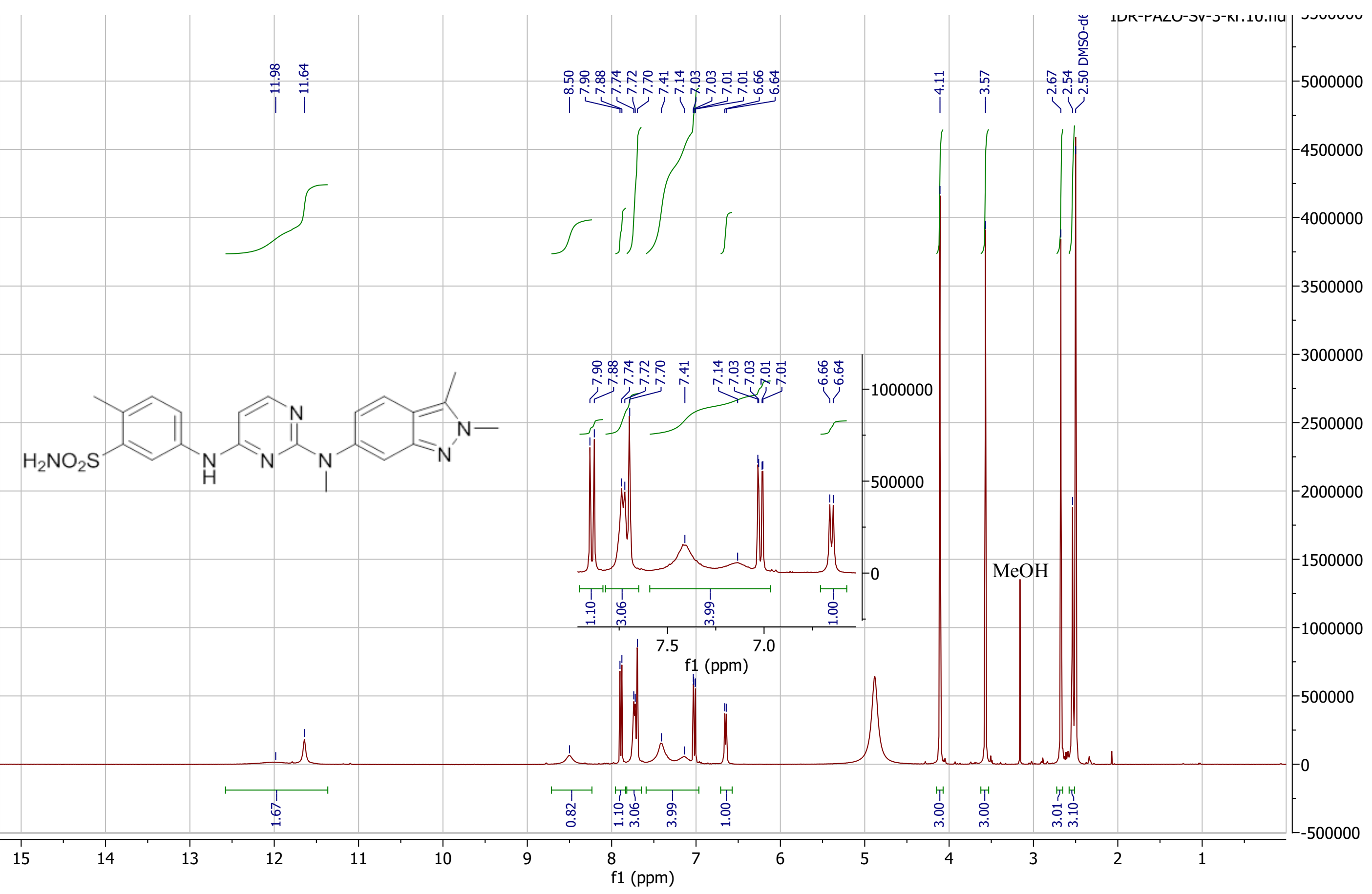

Fig. $37{ }^{1} \mathrm{H}$ NMR spectrum (400 MHz, DMSO-d 6 ) of 5-(2-[(2,3-dimethyl-2H-indazol-6-yl)methylamino]pyrimidin-4-ylamino)-2-methylbenzenesulfonamide hydrochloride(17) 


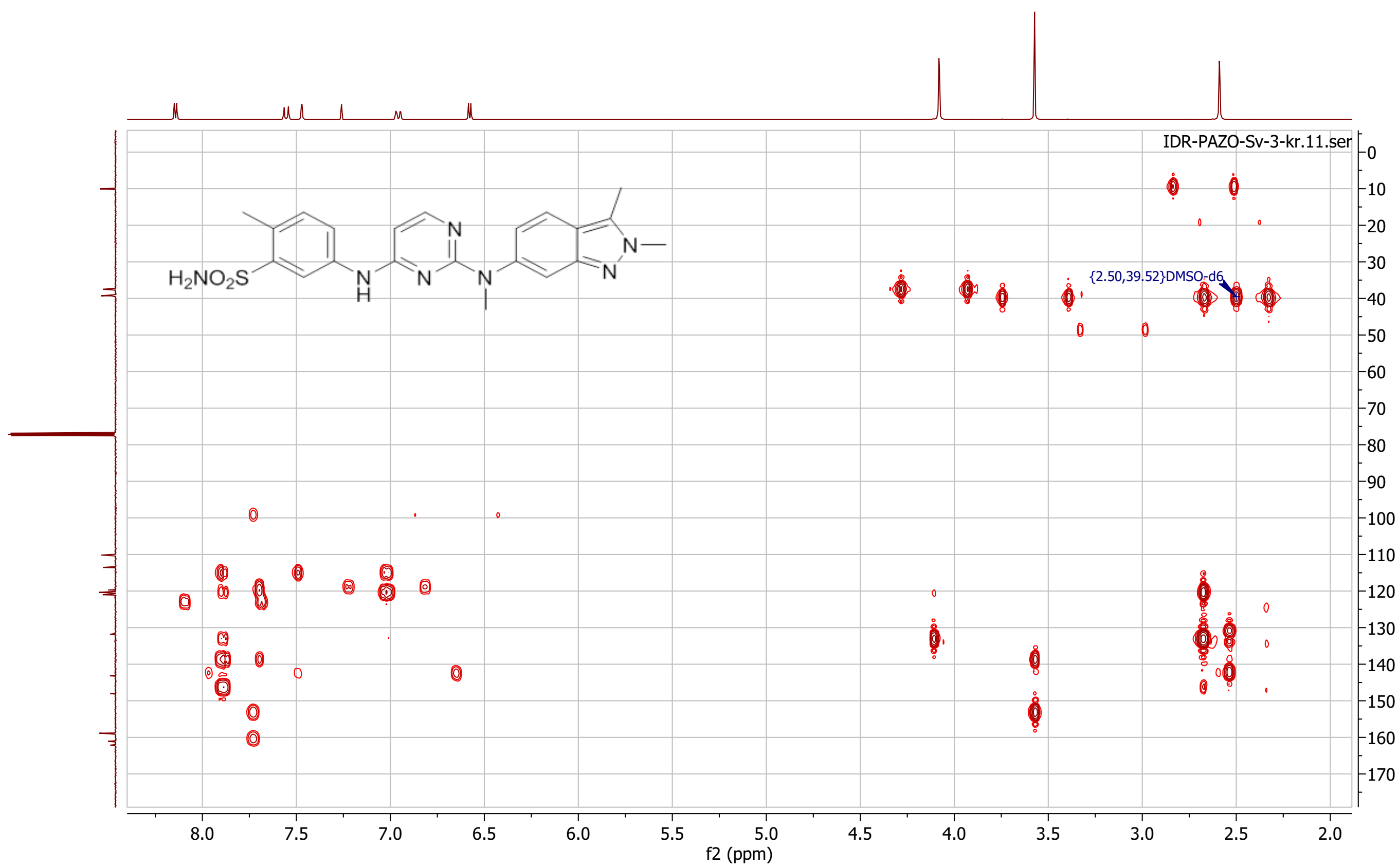

Fig. $38 \mathrm{HMBC}$ spectrum (400 MHz,100 MHz, DMSO-d $\mathrm{d}_{6}$ of 5-(2-[(2,3-dimethyl-2H-indazol-6-yl)methylamino]pyrimidin-4-ylamino)-2-methylbenzenesulfonamide hydrochloride(17) 


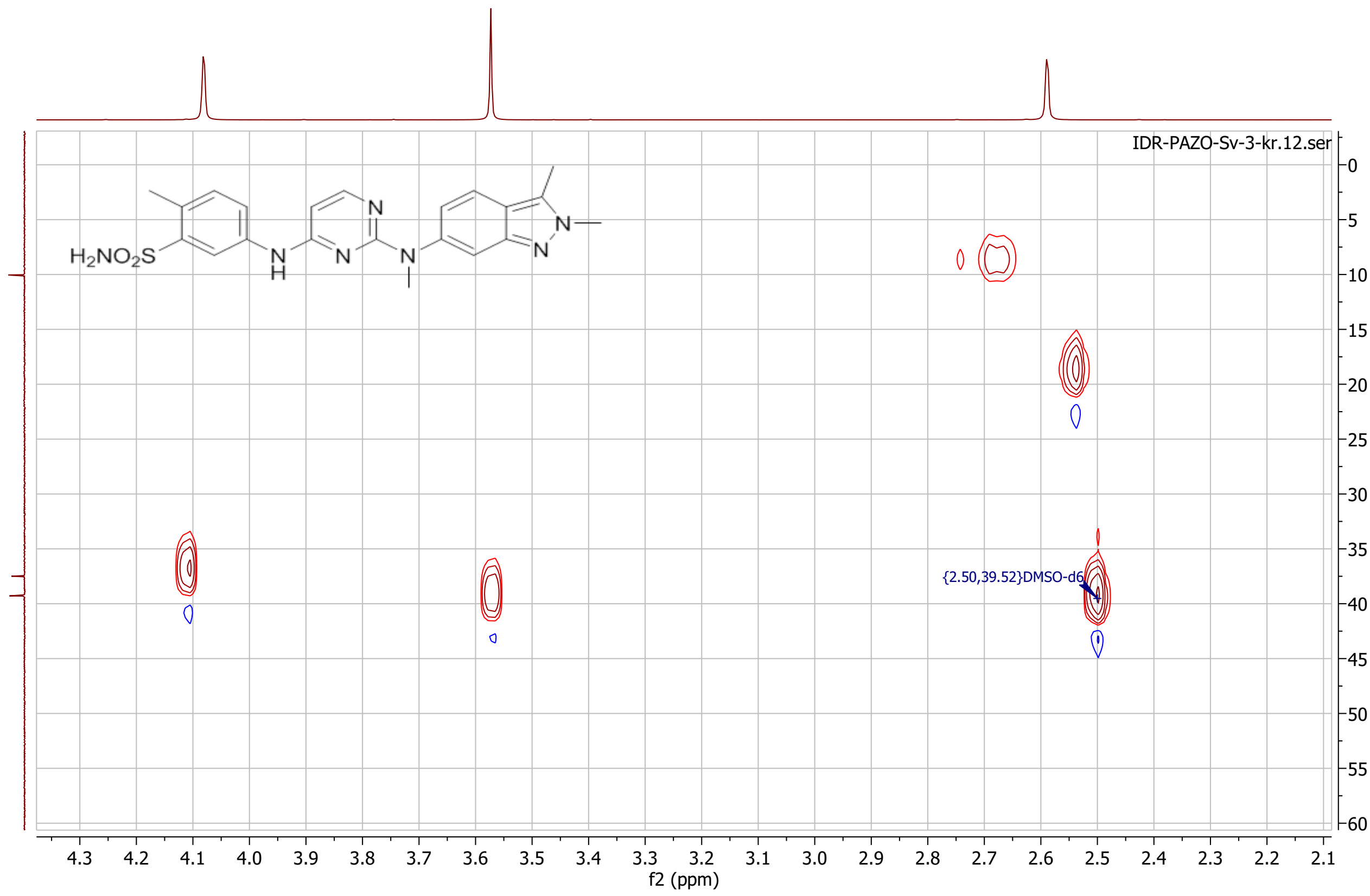

Fig. 39 Part of HSQC spectrum (400 MHz,100 MHz, DMSO-d 6 ) of 5-(2-[(2,3-dimethyl-2H-indazol-6-yl)methylamino]pyrimidin-4-ylamino)-2methylbenzenesulfonamide hydrochloride(17) 


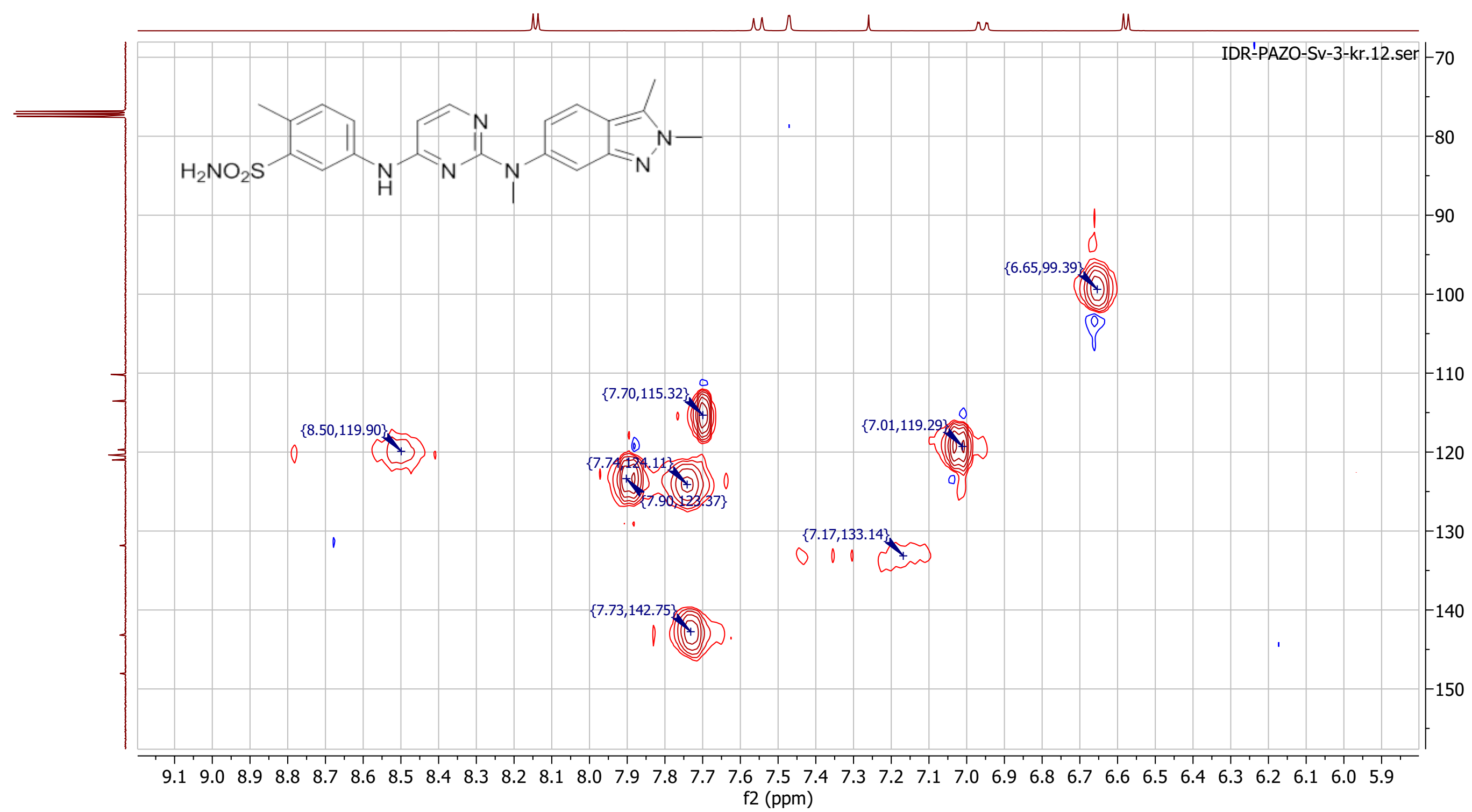

Fig.40 Part of HSQC spectrum (400 MHz,100 MHz, DMSO-d 6 ) of 5-(2-[(2,3-dimethyl-2H-indazol-6-yl)methylamino]pyrimidin-4ylamino)-2-methylbenzenesulfonamide hydrochloride(17) 


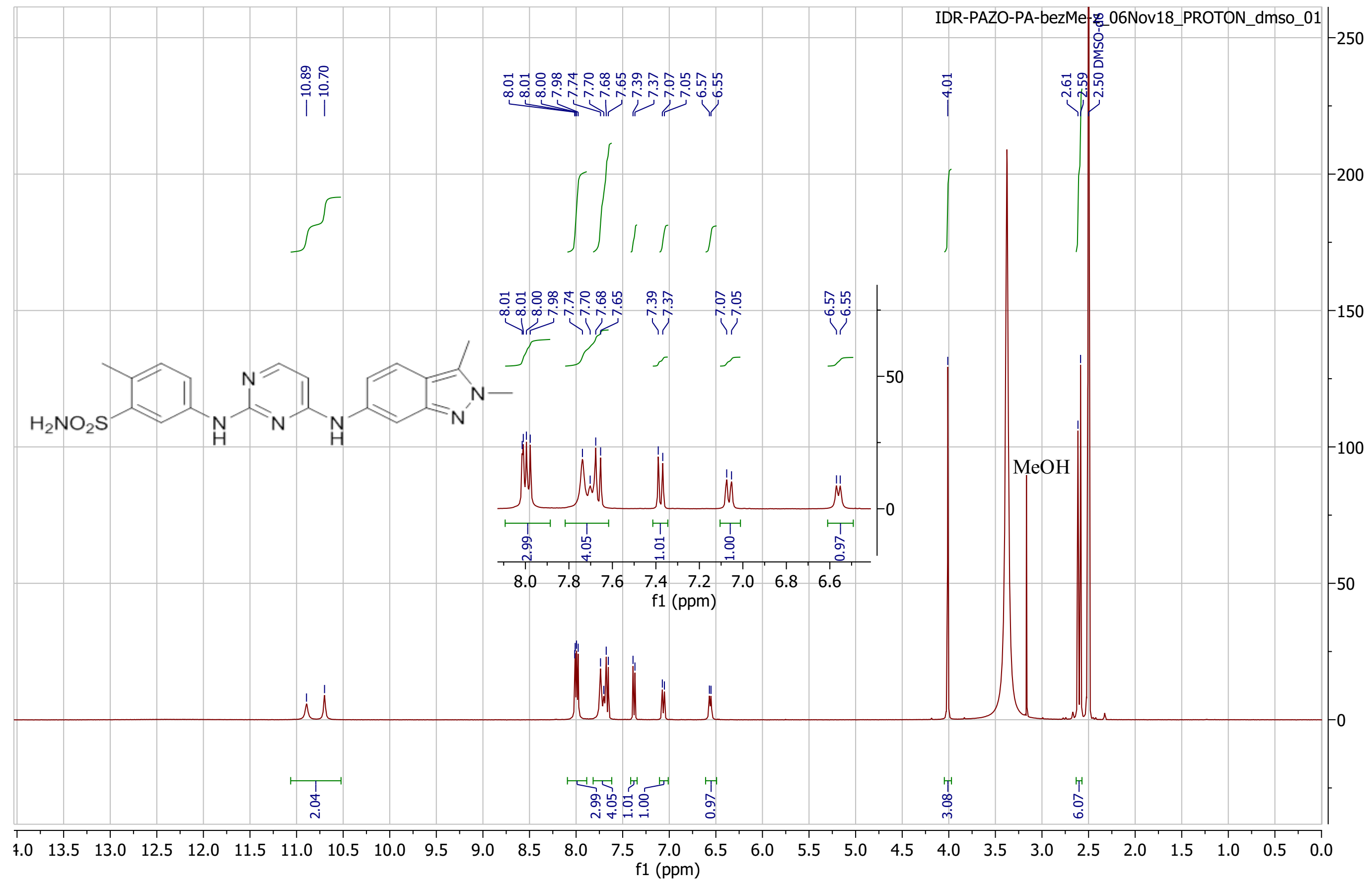

Fig. $41{ }^{1} \mathrm{H}-\mathrm{NMR}$ spectrum (300MHz, DMSO) of 5-[4-[(2,3-dimethyl-2H-indazol-6-ylamino]pyrimidin-2-ylamino]-2-methylbenzene sulfonamide (18) 


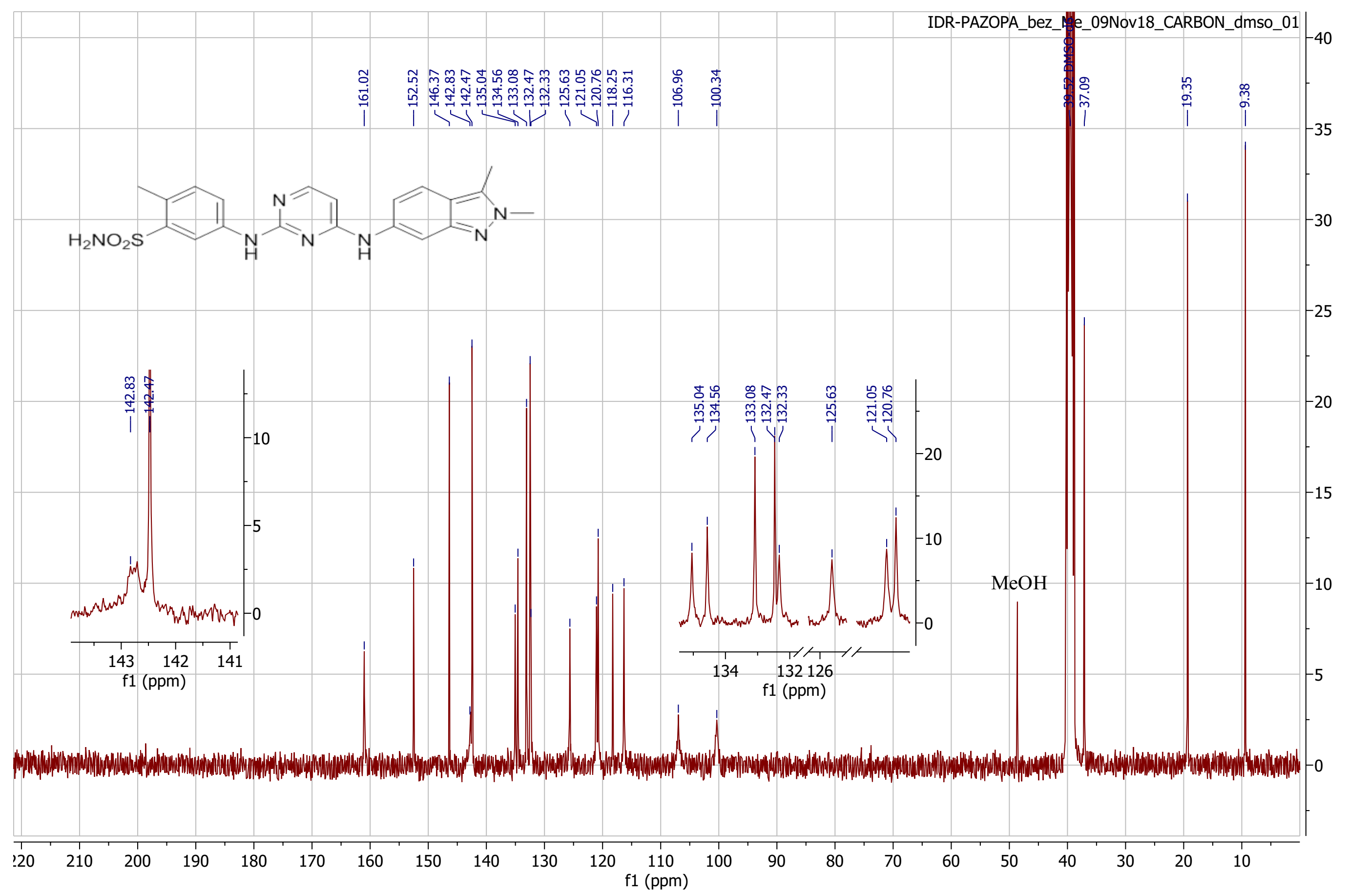

Fig. $42{ }^{13} \mathrm{C}$ NMR spectrum (100MHz, DMSO-d 6 ) of 5-[4-[(2,3-dimethyl-2H-indazol-6-ylamino]pyrimidin-2-ylamino]-2-methylbenzene sulfonamide (18) 
U

$\mu$

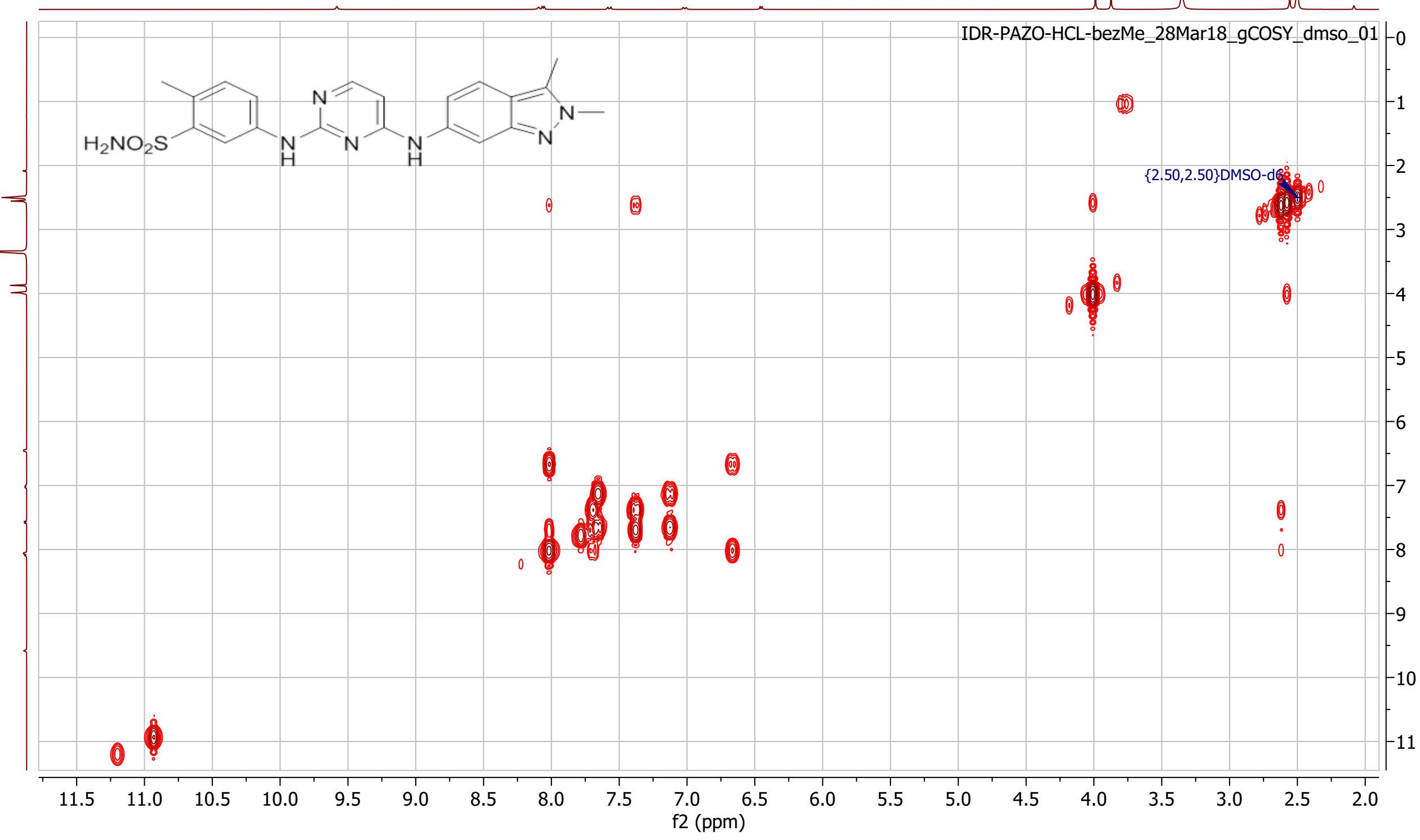

Fig. 43 COSY spectrum $\left(400 \mathrm{MHz}\right.$, DMSO-d $\left.\mathrm{d}_{6}\right)$ of 5-[4-[(2,3-dimethyl-2H-indazol-6-ylamino]pyrimidin-2-ylamino]-2-methylbenzene sulfonamide (18) 
U

$\mu$

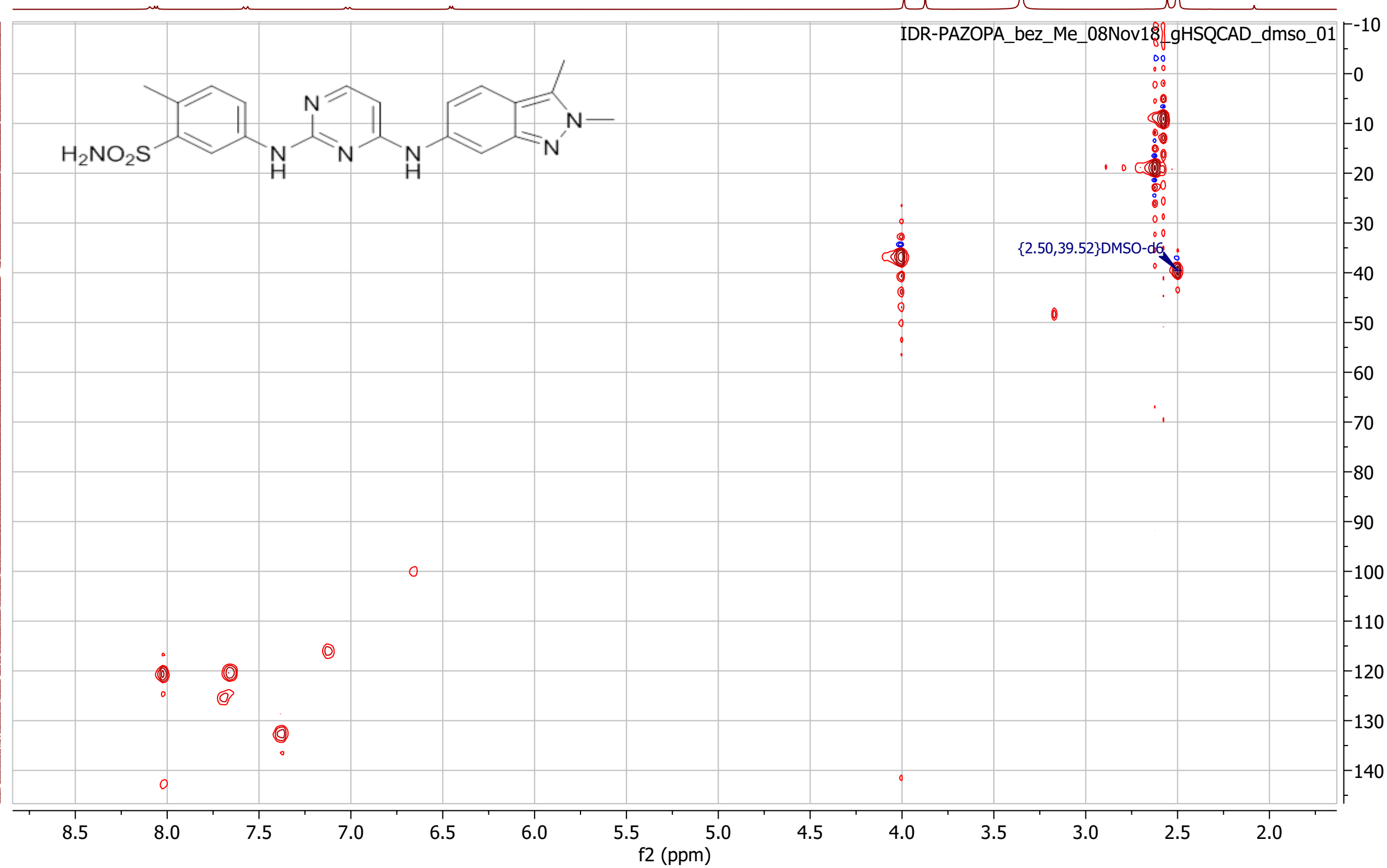

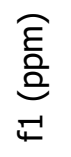




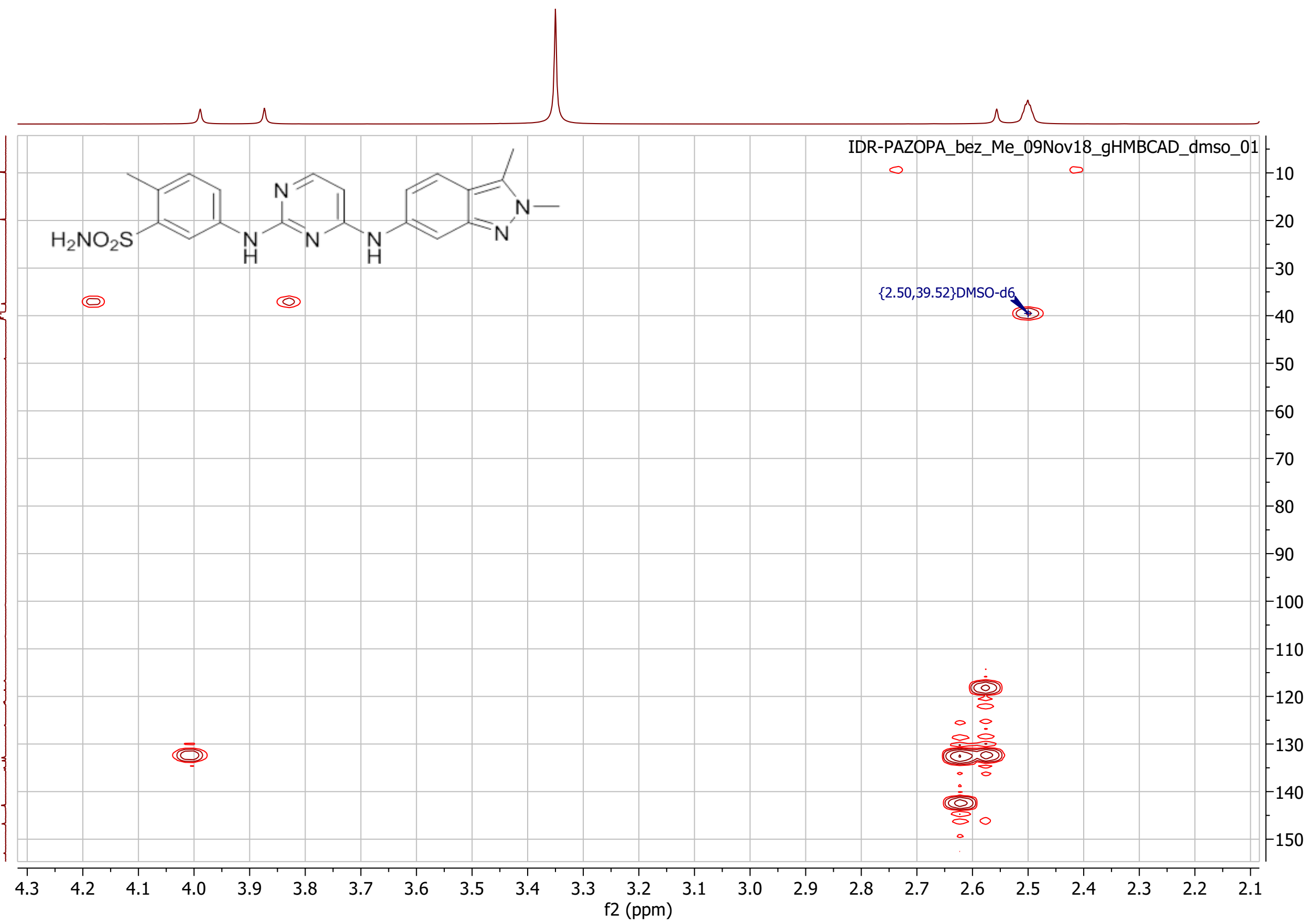

Fig.45 Part of HMBC spectrum (400MHz, $100 \mathrm{MHz}$, DMSO-d $\left.{ }_{6}\right)$ of 5-[4-[(2,3-dimethyl-2H-indazol-6-ylamino]pyrimidin-2-ylamino]-2-methylbenzene sulfonamide (18) 


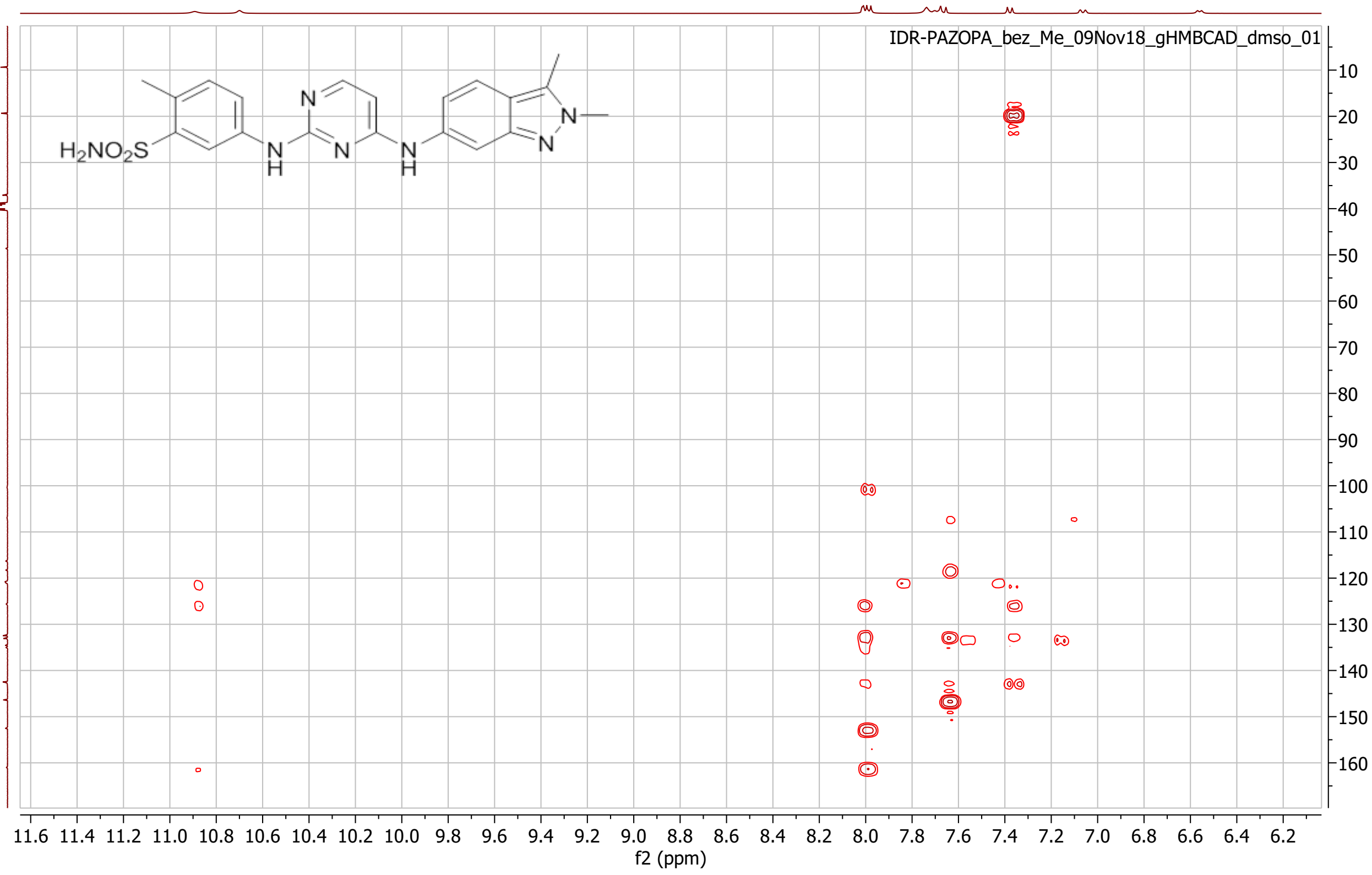




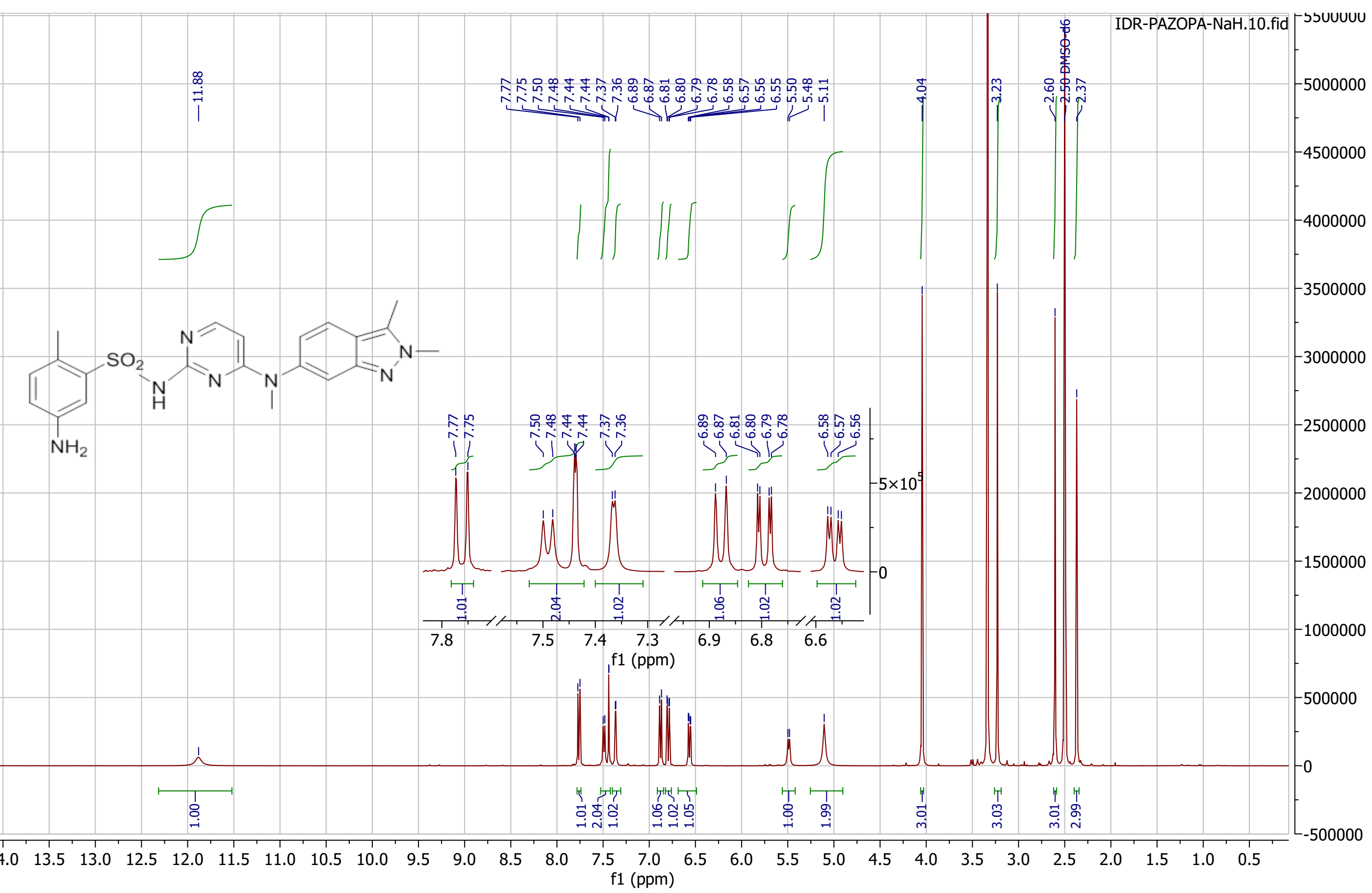

Fig. $47{ }^{1} \mathrm{H}$ NMRspectrum (400 MHz, DMSO-d 6 ) of 5-amino- $N$-[4-[(2,3-dimethyl-2H-indazol-6-yl)-methylamino]pyrimidin-2-yl]-2-methylbenzenesulfonamide (19) 


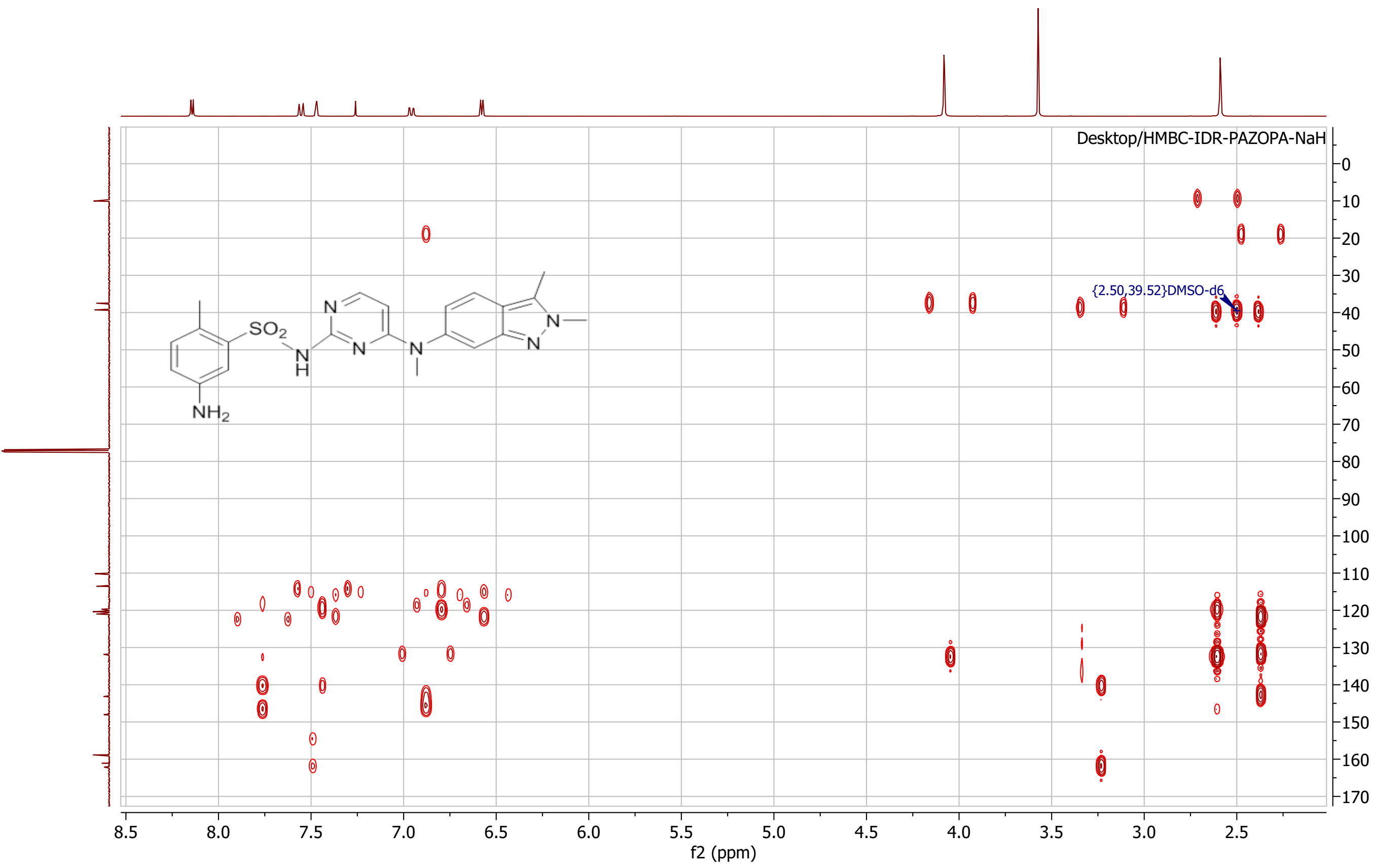

Fig. $48 \mathrm{HMBC}$ spectrum (400 MHz, $\left.100 \mathrm{MHz}, \mathrm{DMSO}-\mathrm{d}_{6}\right)$ of 5-amino- $N$-[4-[(2,3-dimethyl-2H-indazol-6-yl)-methylamino]pyrimidin-2-yl]-2methylbenzenesulfonamide (19) 


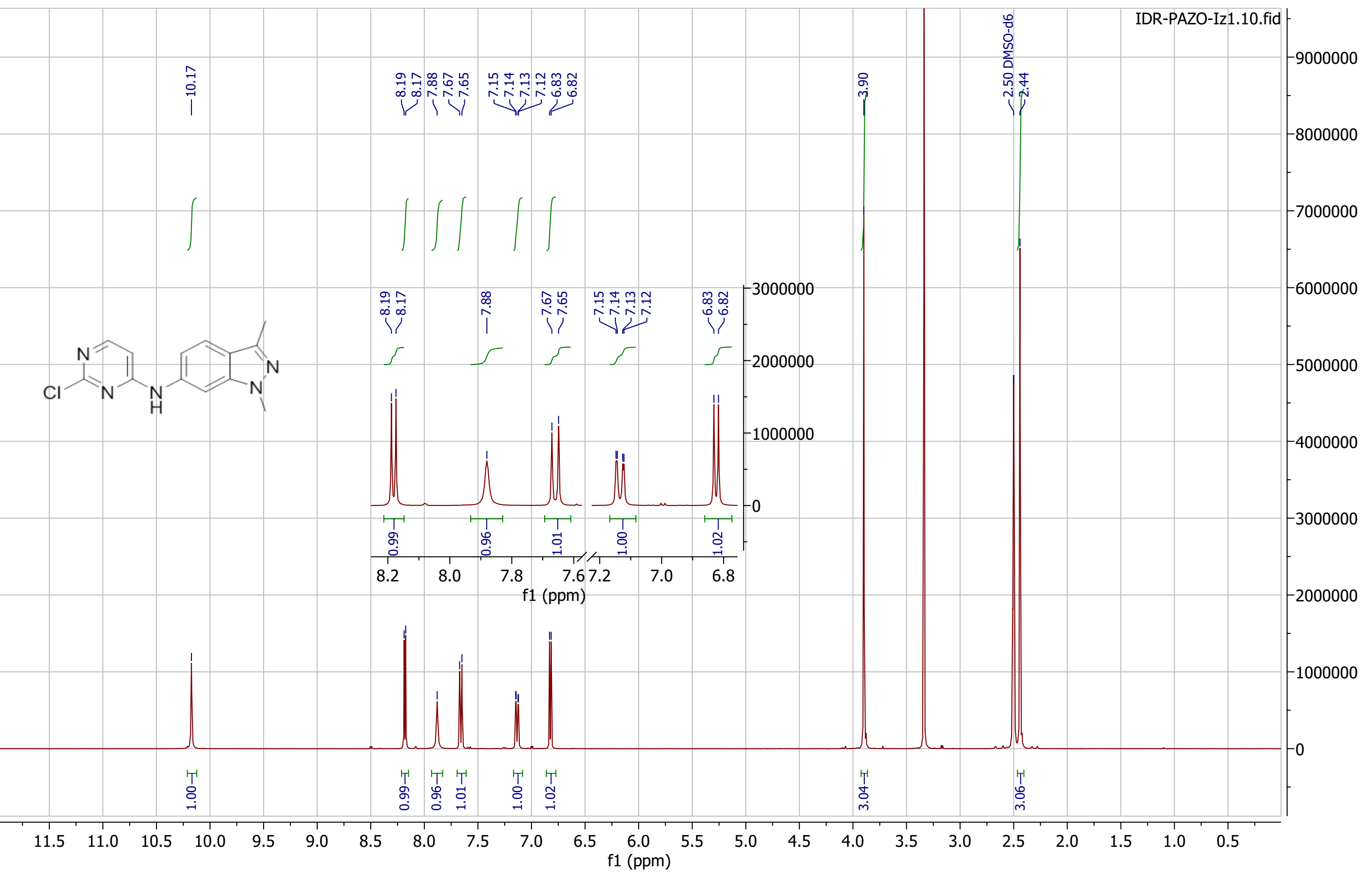

Fig. $49{ }^{1} \mathrm{H}$ NMR spectrum (300 MHz, DMSO-d 6 ) of (2-chloropyrimidin-4-yl)-(1,3-dimethyl-1H-indazol-6-yl)amine (21) 


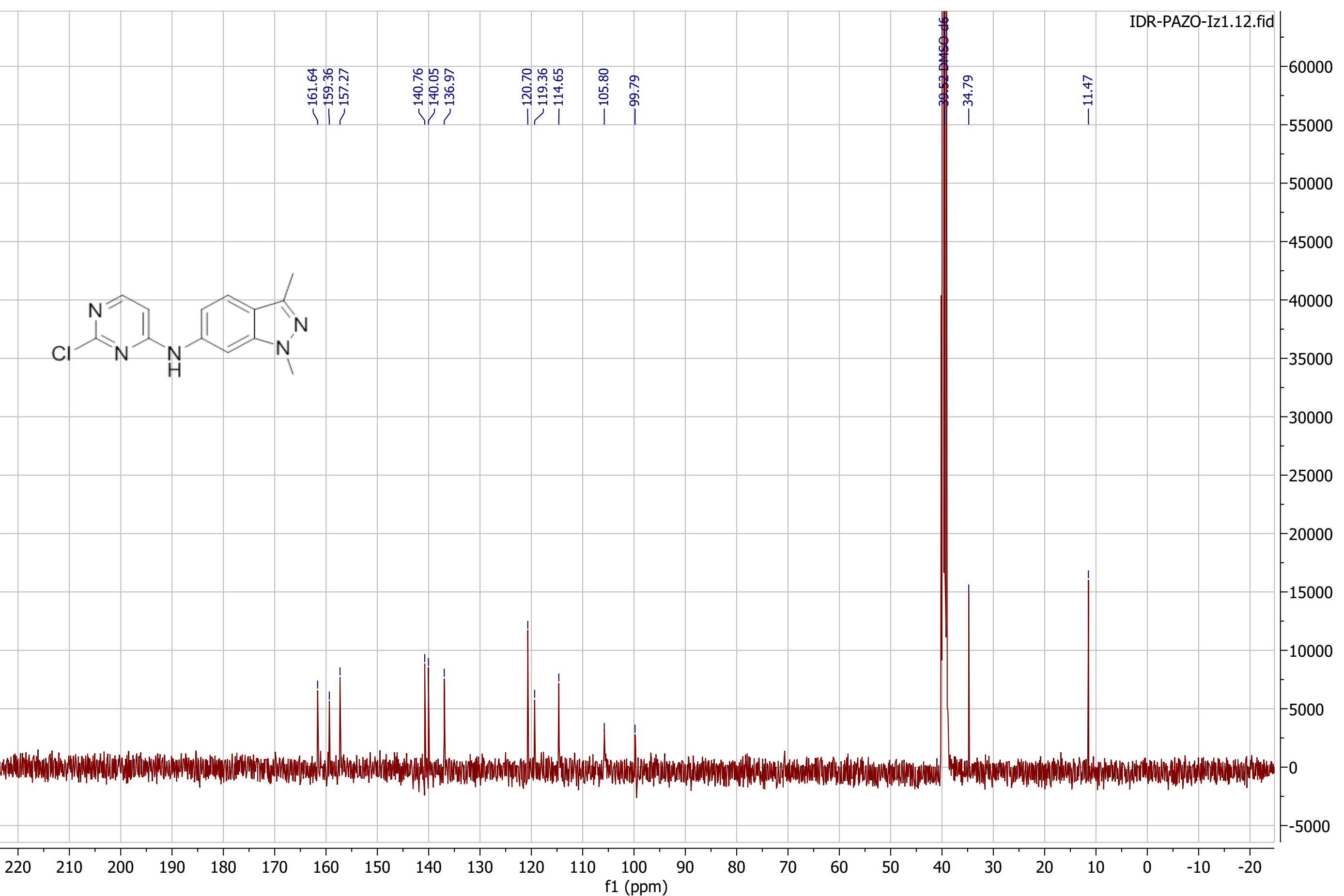

Fig. $50{ }^{13} \mathrm{C}$ NMR spectrum (100 MHz, DMSO-d 6 of (2-chloropyrimidin-4-yl)-(1,3-dimethyl-1 $H$-indazol-6-yl)amine (21) 


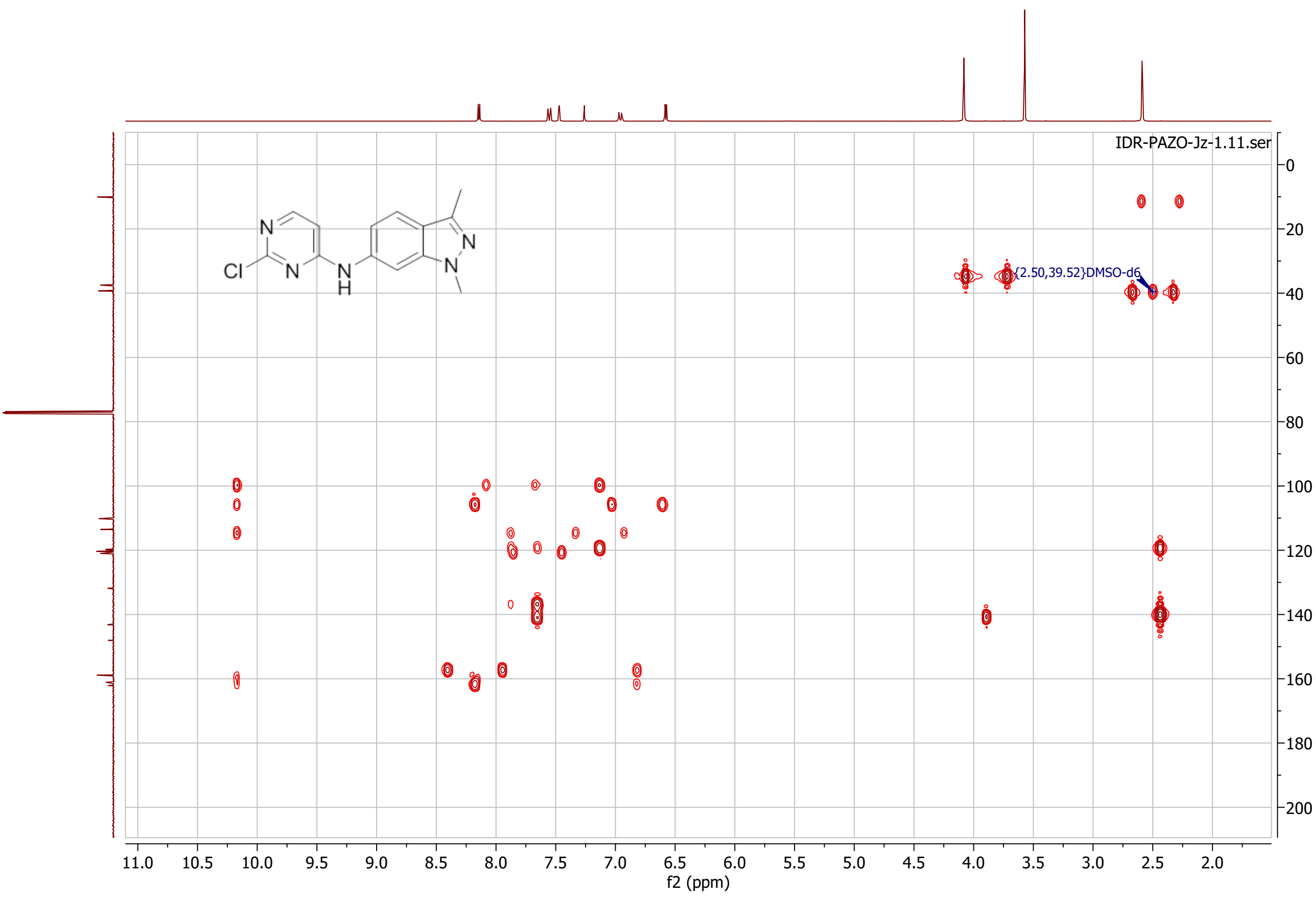

Fig. 51 HMBC spectrum (400 MHz, 100 MHz, DMSO-d $)$ of (2-chloropyrimidin-4-yl)-(1,3-dimethyl-1H-indazol-6-yl)amine (21) 


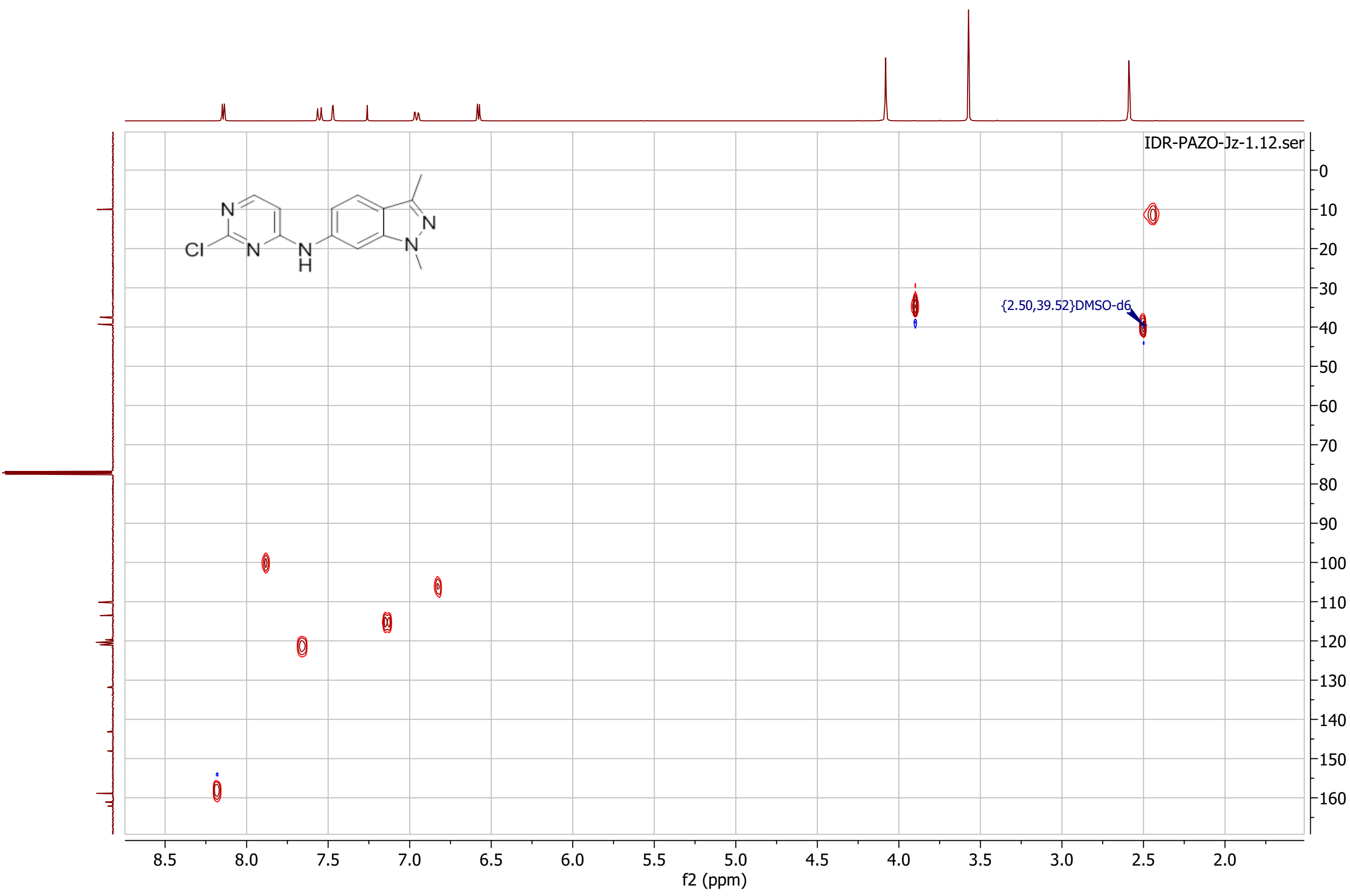

Fig. 52 HSQC spectrum (400 MHz, 100 MHz, DMSO-d ${ }_{6}$ ) of (2-chloropyrimidin-4-yl)-(1,3-dimethyl-1H-indazol-6-yl)amine (21) 


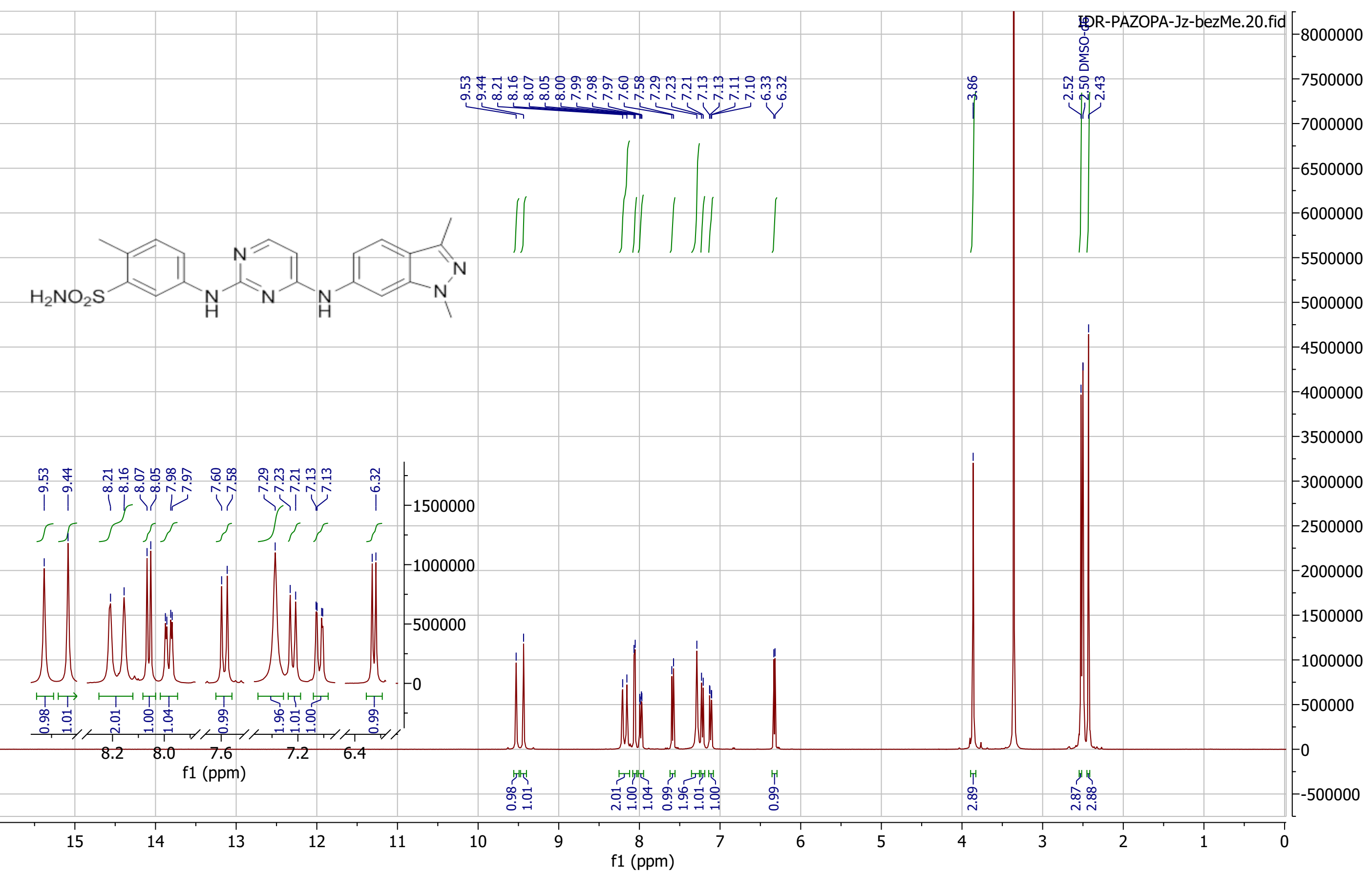

Fig. $53{ }^{1} \mathrm{H}$ NMR spectrum (400 MHz, DMSO-d 6 ) of 5-[4-(1,3-dimethyl-1H-indazol-6-ylamino)pyrimidin-2-ylamino]-2-methylbenzene sulfonamide (22) 


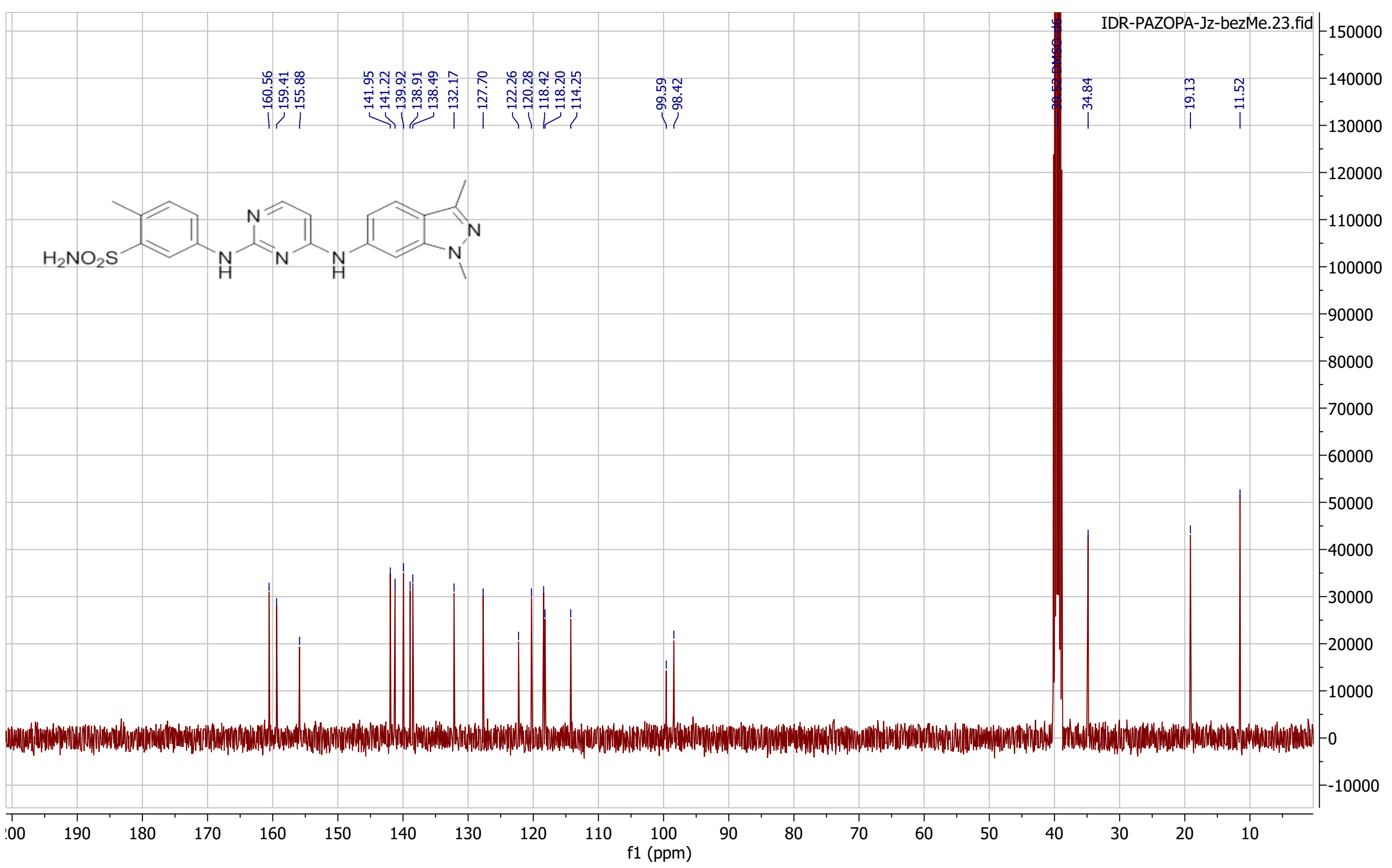

Fig. $54{ }^{13} \mathrm{C}$ NMR spectrum (100 MHz, DMSO-d $\left.\mathrm{d}_{6}\right)$ of 5-[4-(1,3-dimethyl-1H-indazol-6-ylamino)pyrimidin-2-ylamino]-2-methylbenzene sulfonamide (22) 


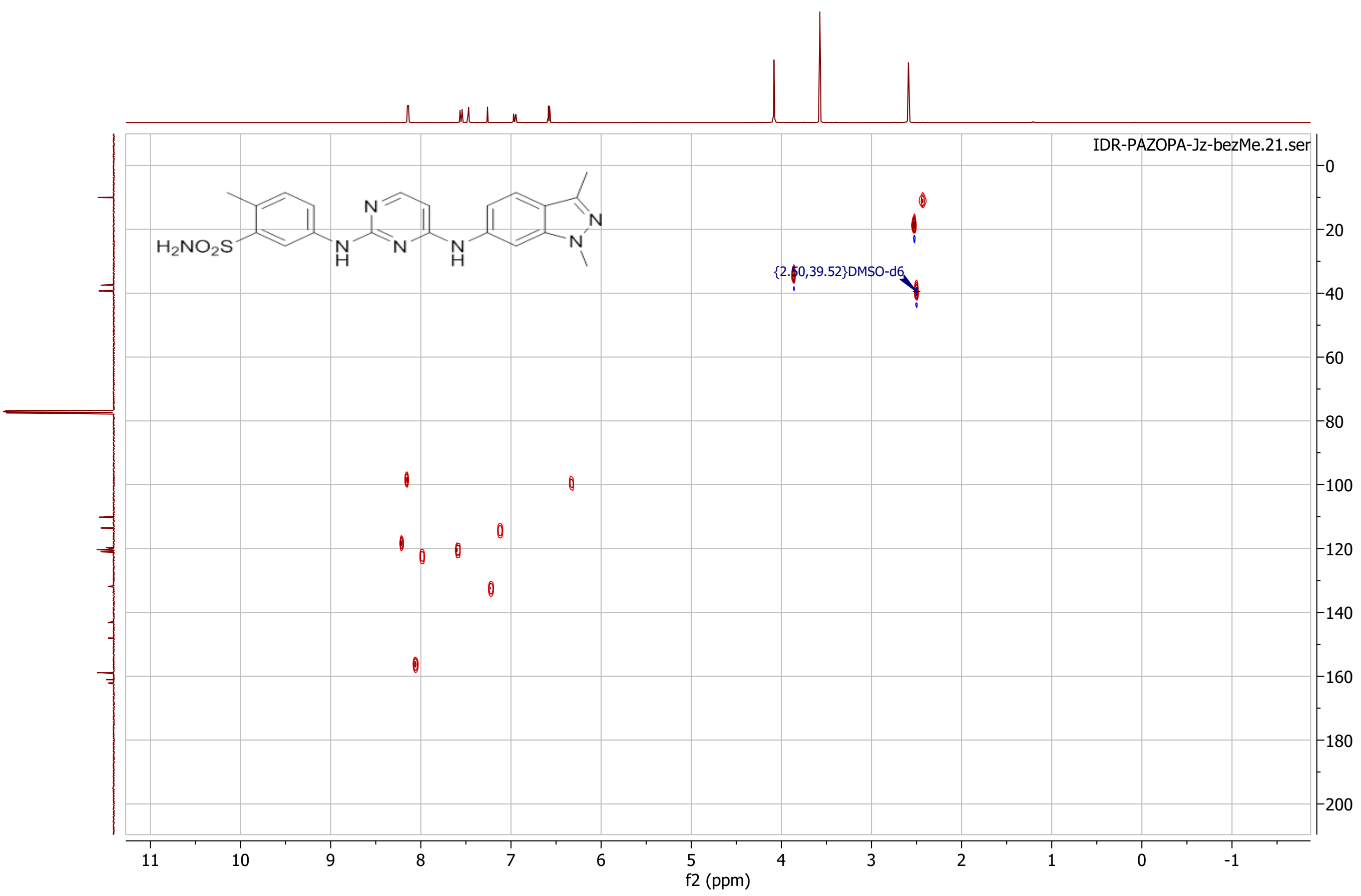

Fig. 55 HSQC spectrum (400 MHz,100 MHz, DMSO-d ${ }_{6}$ ) of 5-[4-(1,3-dimethyl-1H-indazol-6-ylamino)pyrimidin-2-ylamino]-2-methylbenzene sulfonamide (22) 


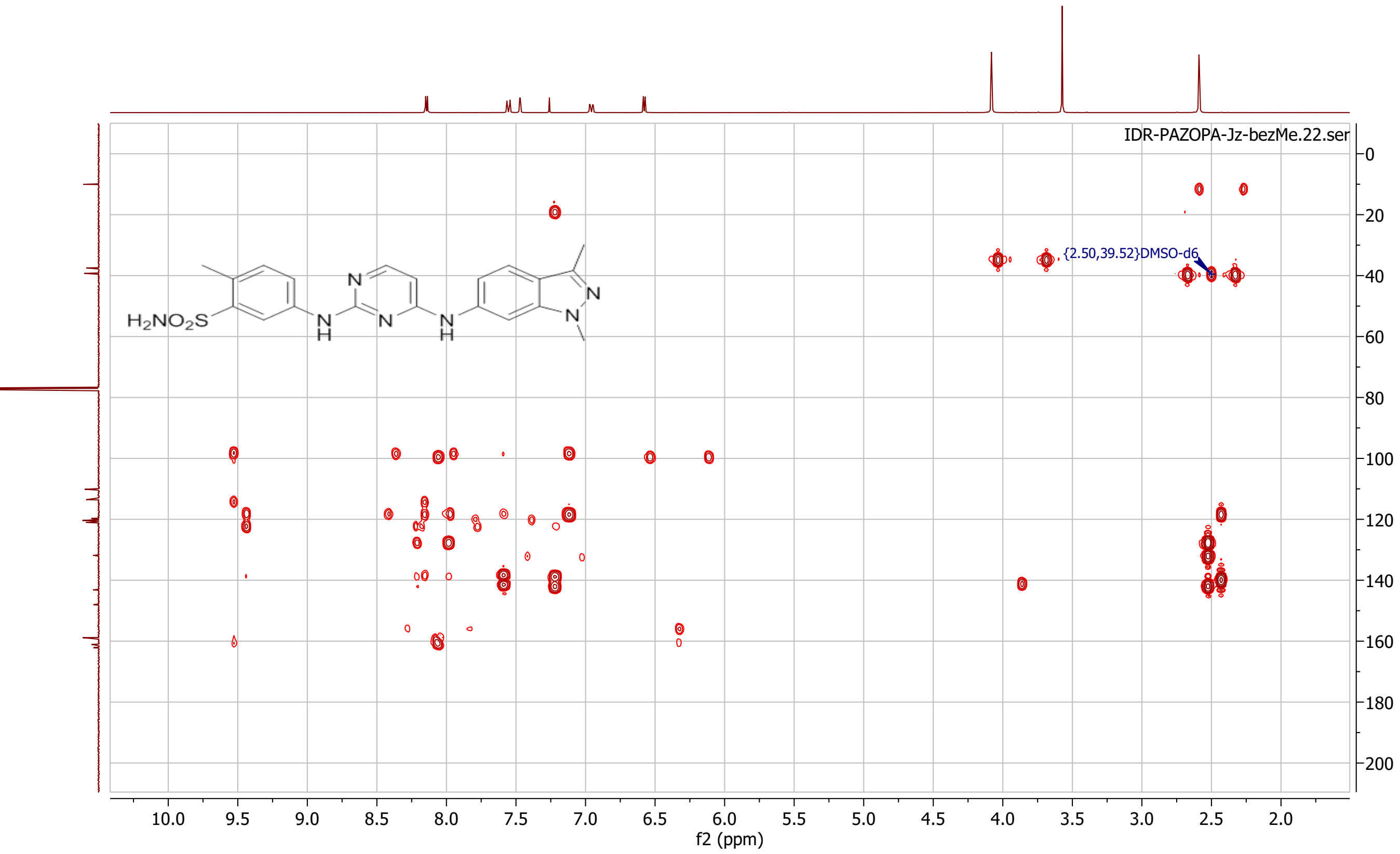

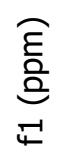

Fig. 56 HMBC spectrum (400 MHz,100 MHz, DMSO-d ${ }_{6}$ ) of 5-[4-(1,3-dimethyl-1H-indazol-6-ylamino)pyrimidin-2-ylamino]-2-methylbenzene sulfonamide (22) 


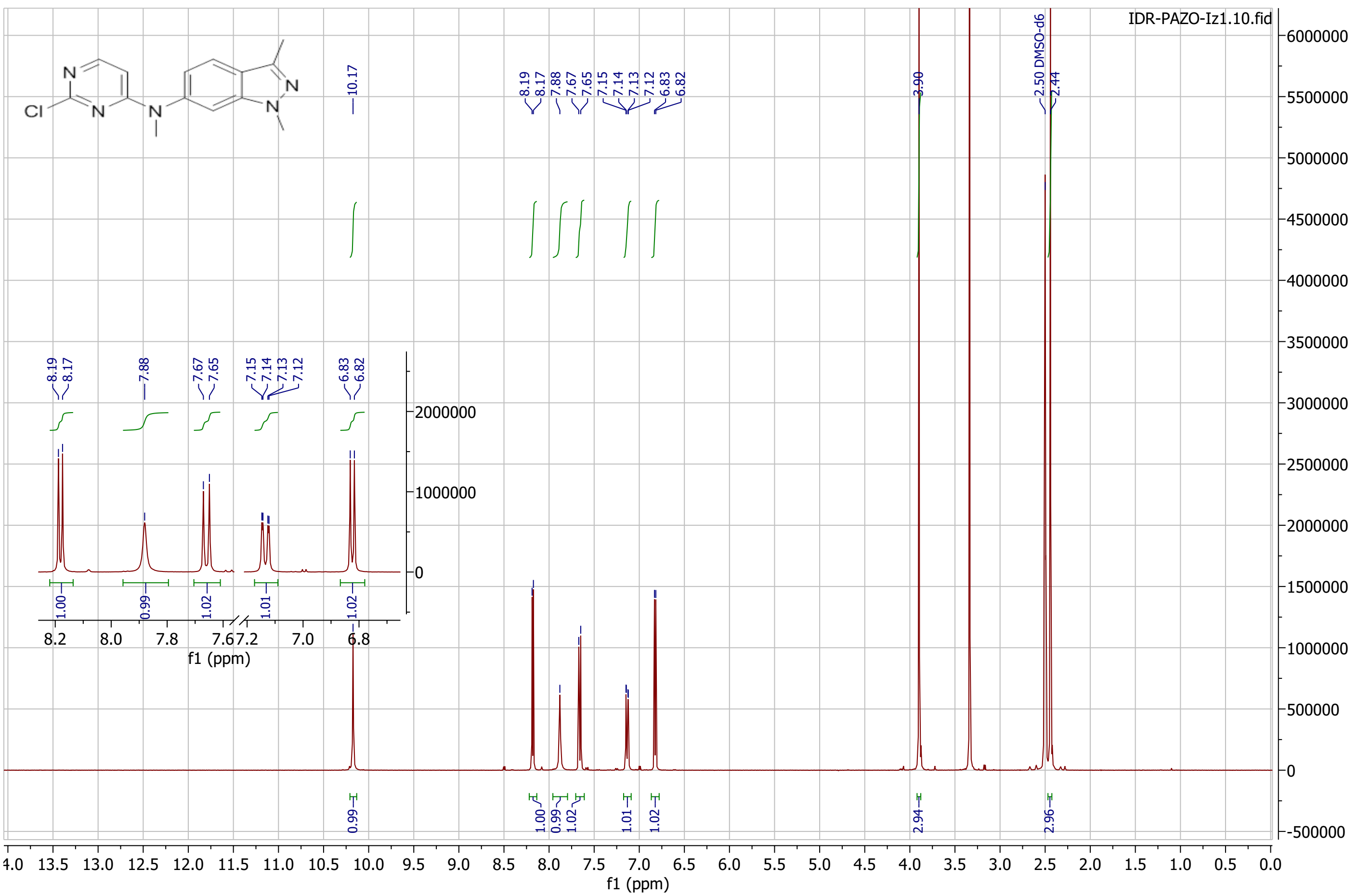

Fig. $57{ }^{1} \mathrm{H}$ NMR spectrum (300 MHz, $\mathrm{CDCl}_{3}$ ) of (2-chloropyrimidin-4-yl)-(1,3-dimethyl-1H-indazol-6-yl)methylamine (23) 


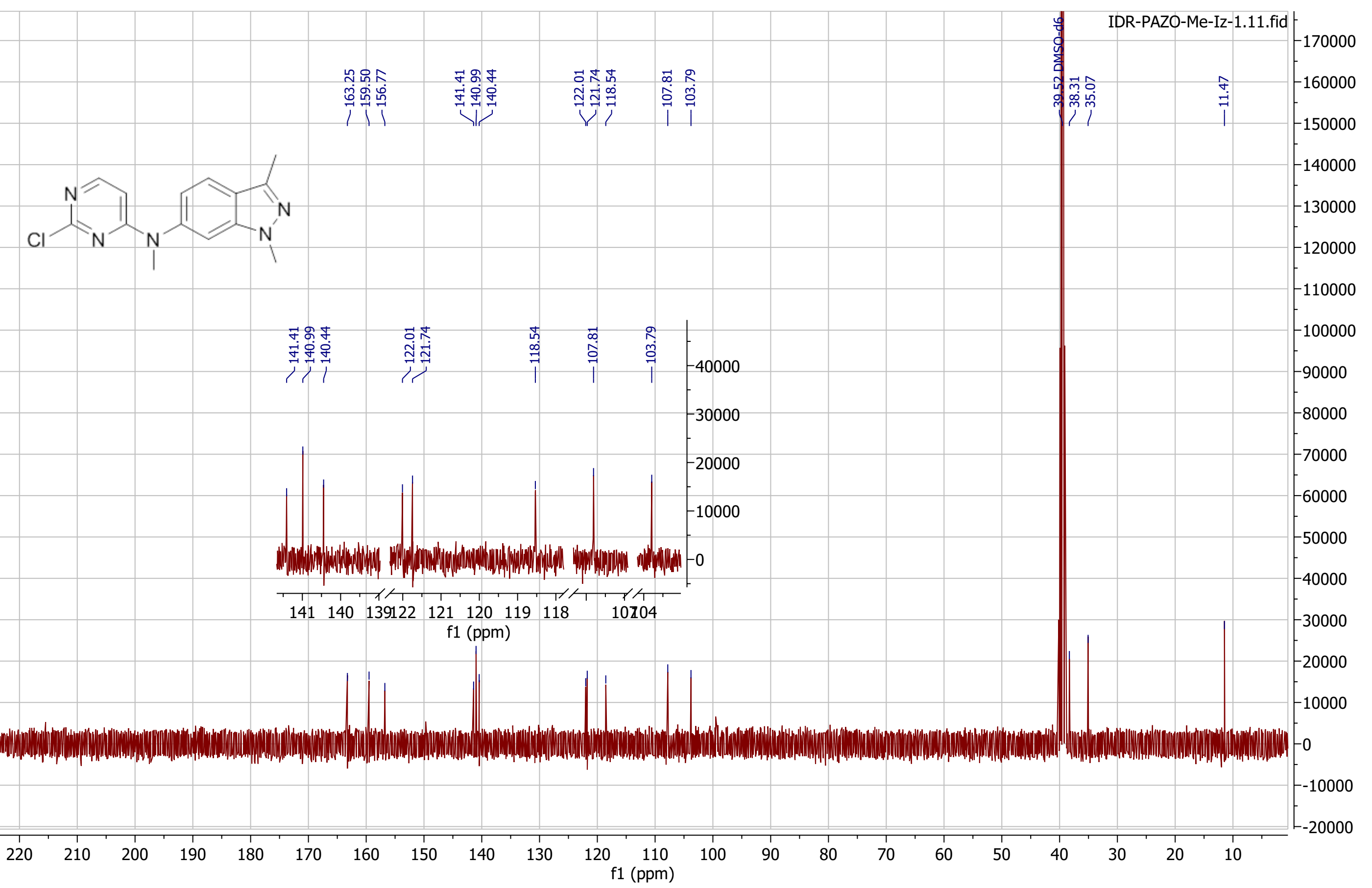

Fig. $58{ }^{13} \mathrm{C}$ NMR spectrum (100 MHz, DMSO-d 6 ) of (2-chloropyrimidin-4-yl)-(1,3-dimethyl- $1 H$-indazol-6-yl)methylamine (23) 


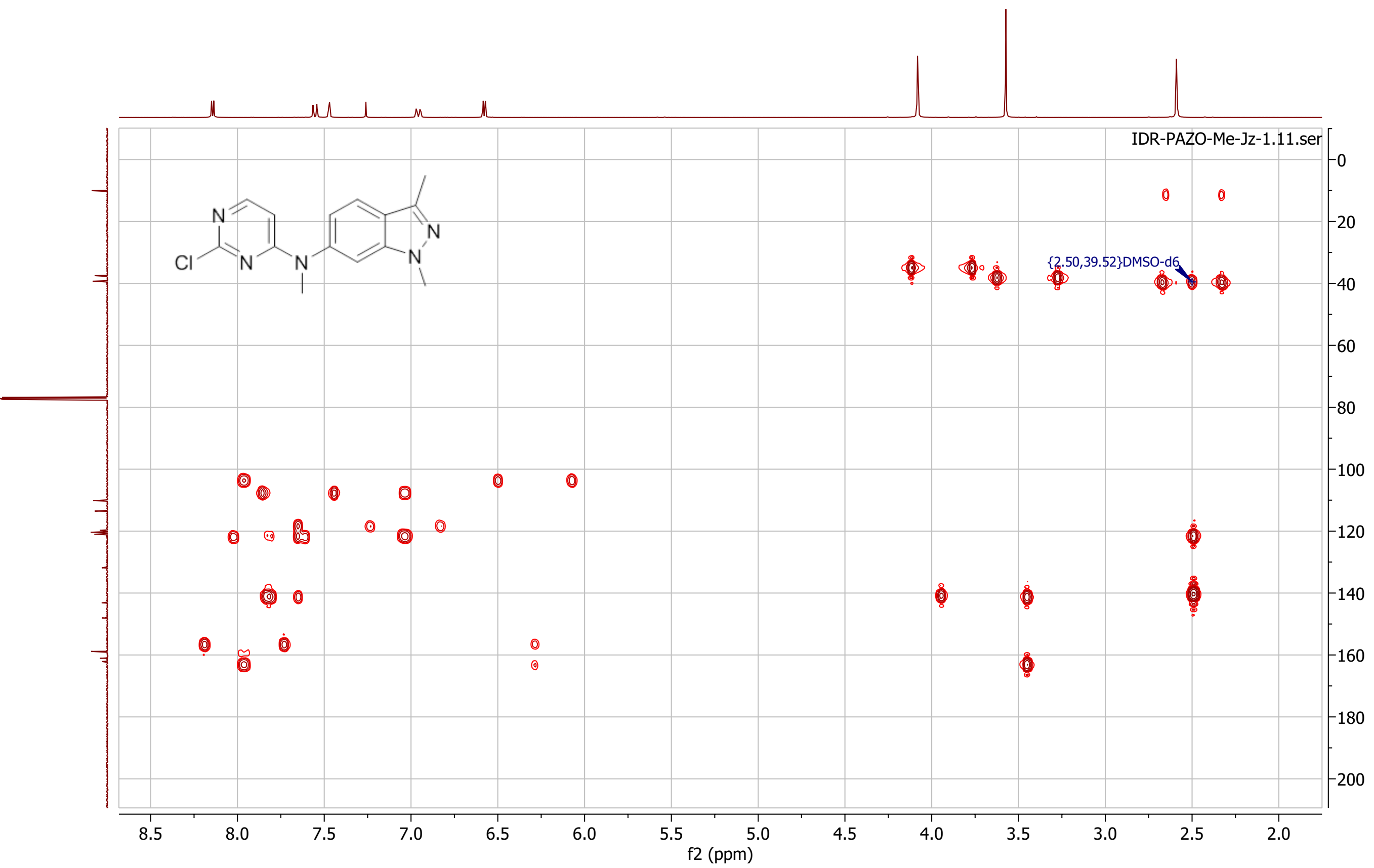

Fig. 59 HMBC spectrum (400 MHz, 100 MHz, DMSO-d ${ }_{6}$ ) of (2-chloropyrimidin-4-yl)-(1,3-dimethyl-1H-indazol-6-yl)methylamine (23) 


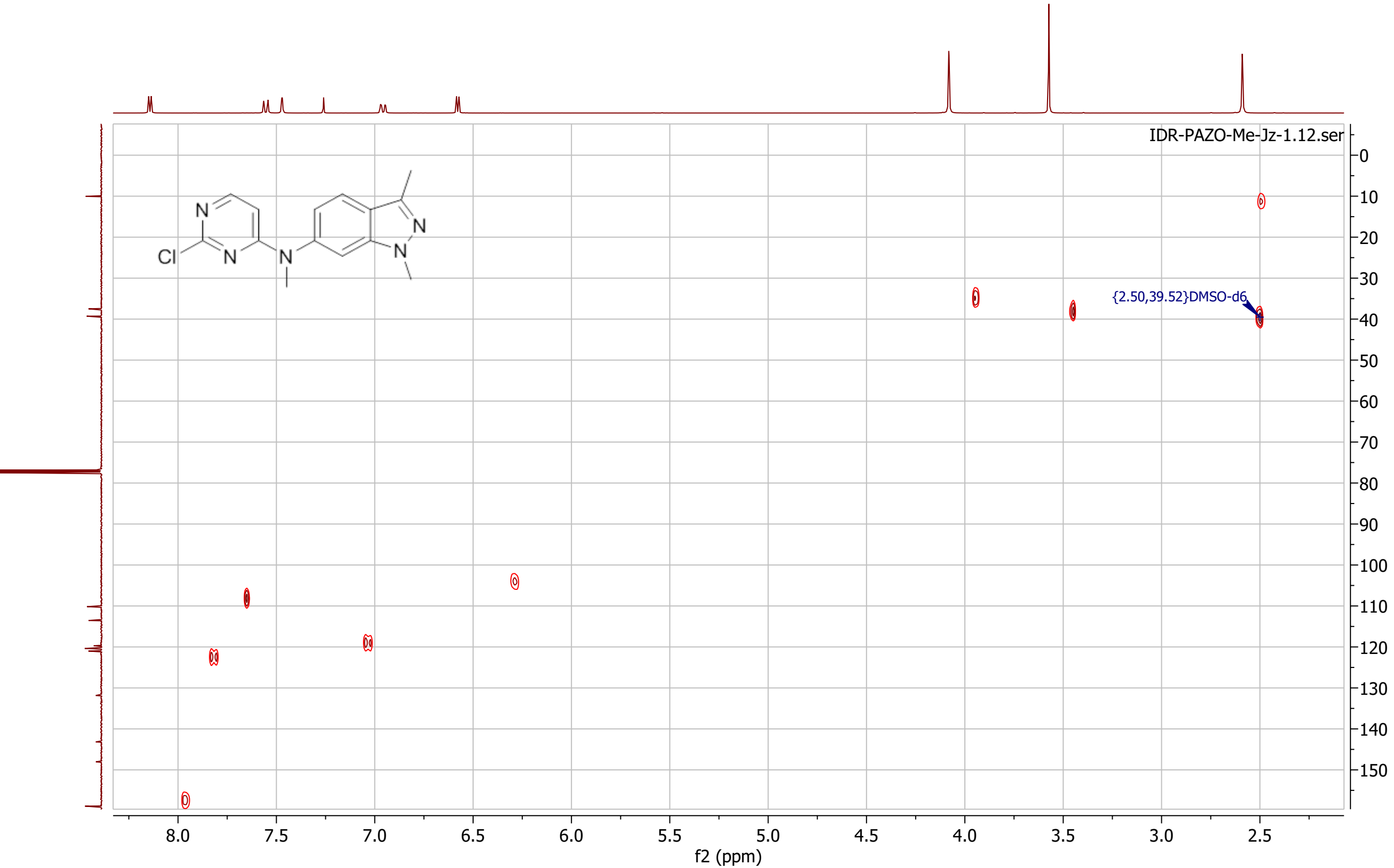

Fig. 60 HSQC spectrum (400 MHz, 100 MHz, DMSO-d d $_{6}$ of (2-chloropyrimidin-4-yl)-(1,3-dimethyl- $1 H$-indazol-6-yl)methylamine (23) 


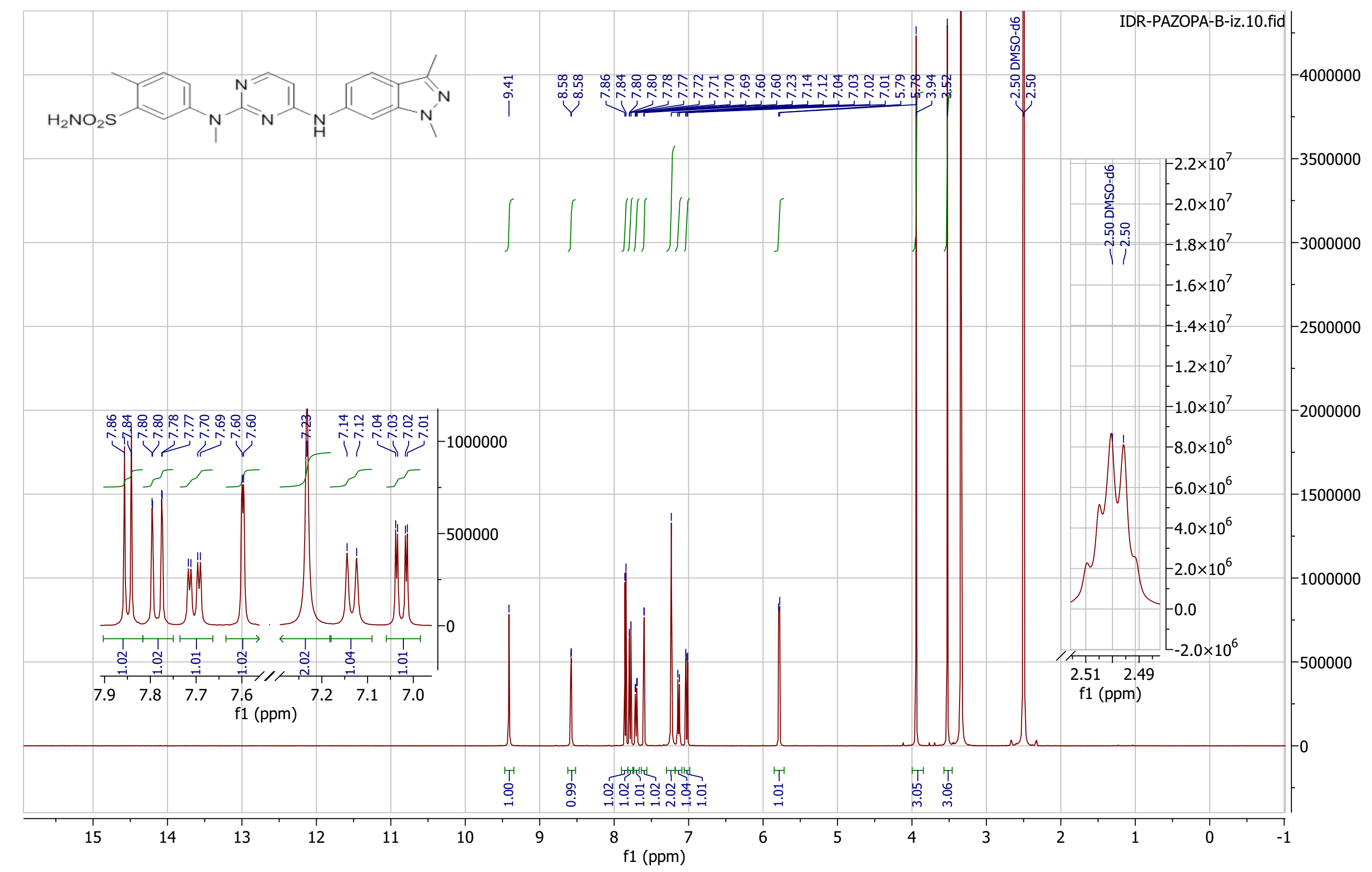

Fig. $61{ }^{1} \mathrm{H}$ NMR spectrum (300 MHz, DMSO-d $\left.{ }_{6}\right)$ of 5-(4-[(1,3-dimethyl-1H-indazol-6-yl)methylamino]pyrimidin-2-ylamino)-2-methylbenzenesulfonamide (25) 


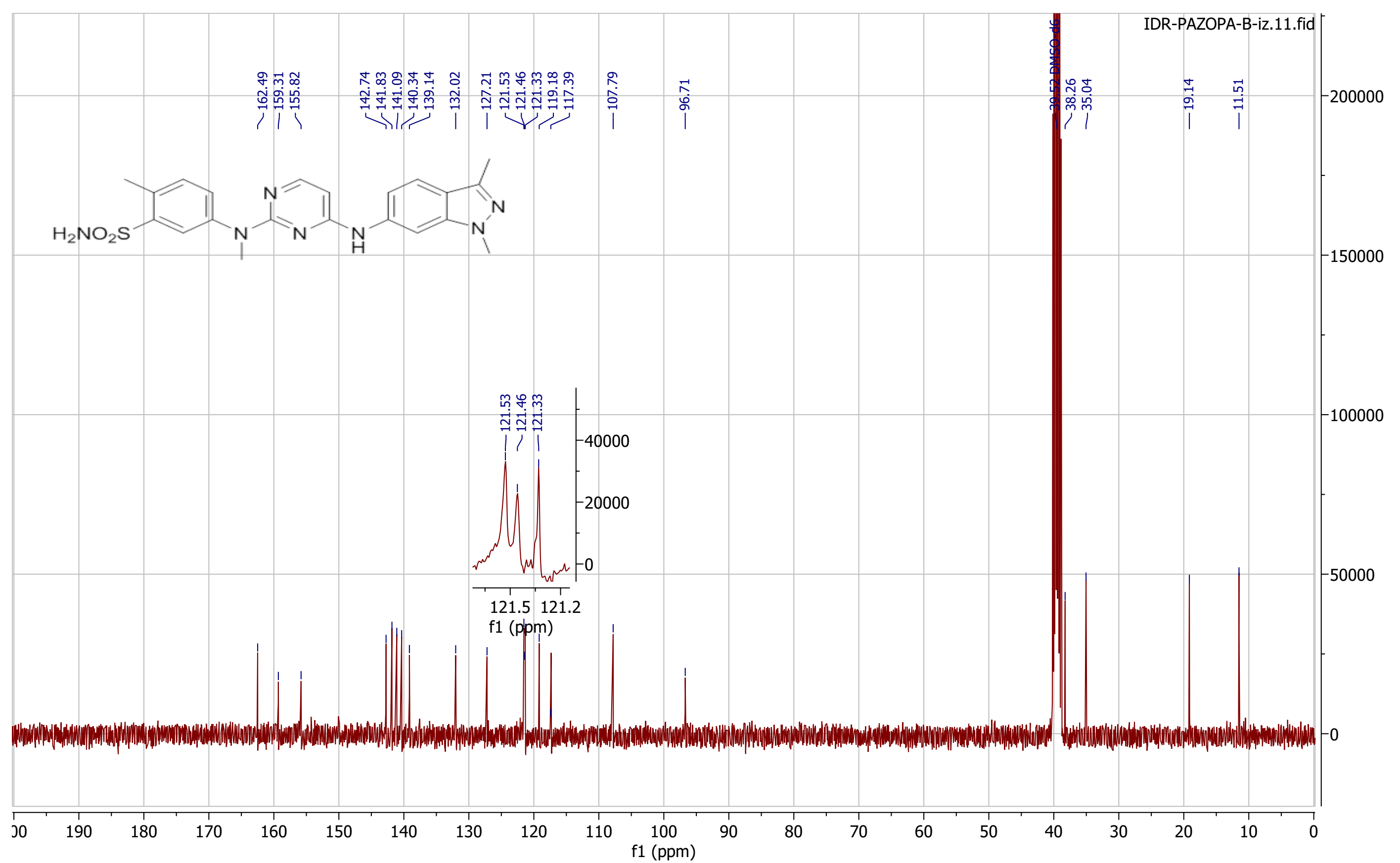

Fig. $62{ }^{13} \mathrm{C}$ NMR spectrum (100 MHz, DMSO-d 6 ) of 5-(4-[(1,3-dimethyl- $1 H$-indazol-6-yl)methylamino]pyrimidin-2-ylamino)-2-methylbenzenesulfonamide (25) 


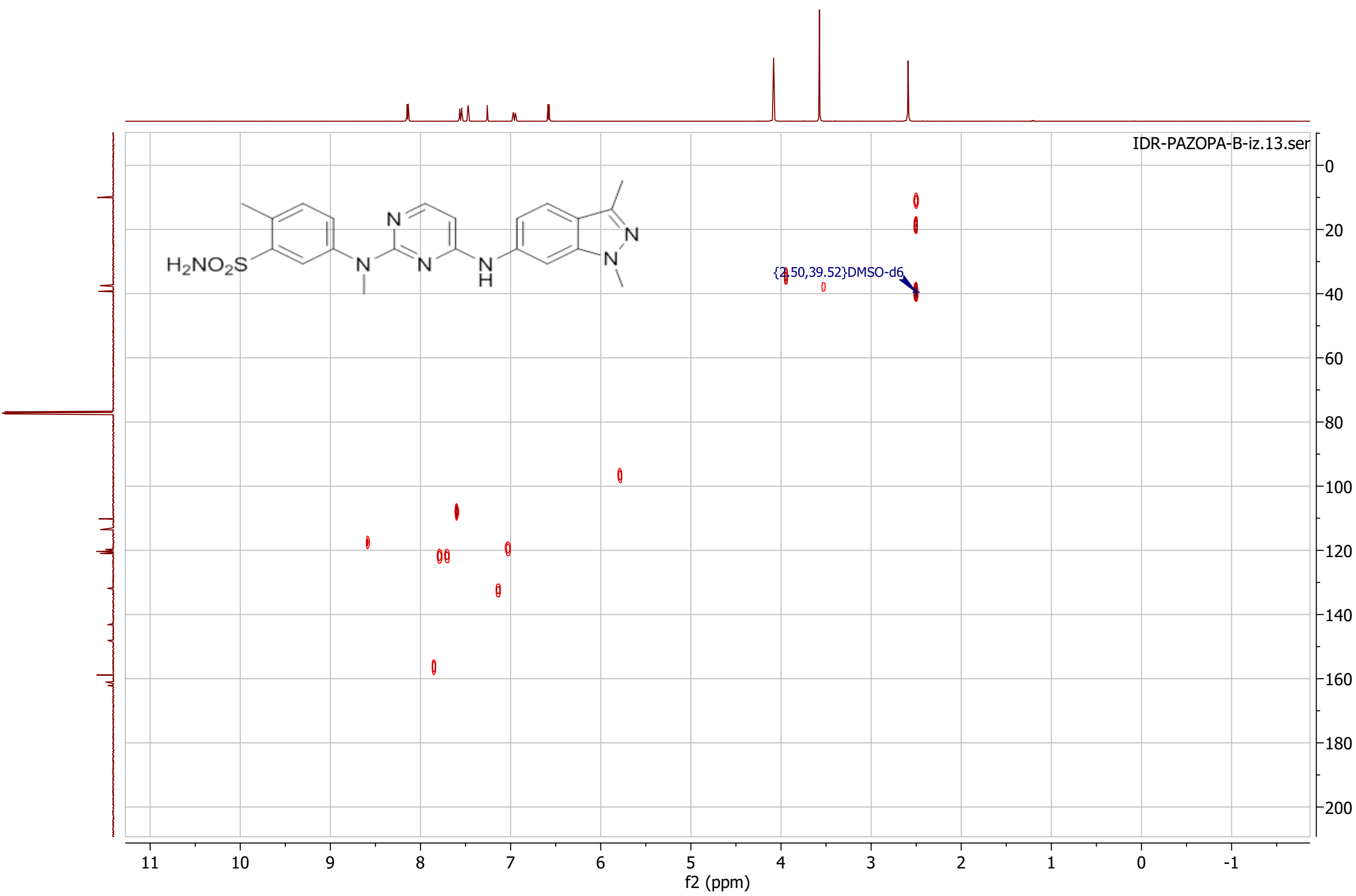

Fig. 63 HSQC spectrum (400 MHz, 100 MHz, DMSO-d $)$ of 5-(4-[(1,3-dimethyl- 1 H-indazol-6-yl)methylamino]pyrimidin-2-ylamino)-2methylbenzenesulfonamide (25) 


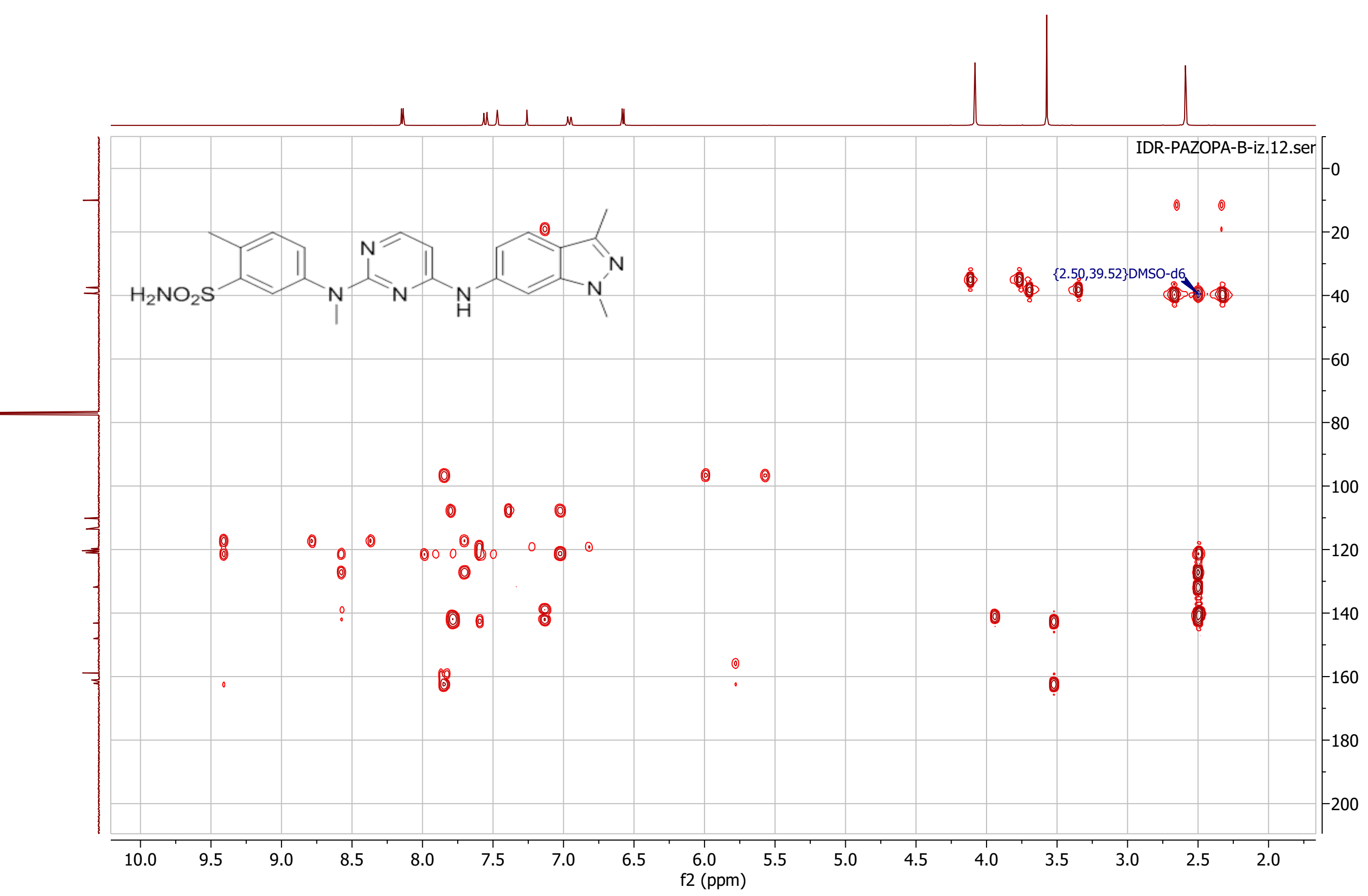

Fig. $64 \mathrm{HMBC}$ spectrum (400 MHz, $100 \mathrm{MHz}$, DMSO-d $\left.{ }_{6}\right)$ of 5-(4-[(1,3-dimethyl- $1 H$-indazol-6-yl)methylamino]pyrimidin-2-ylamino)-2methylbenzenesulfonamide (25) 


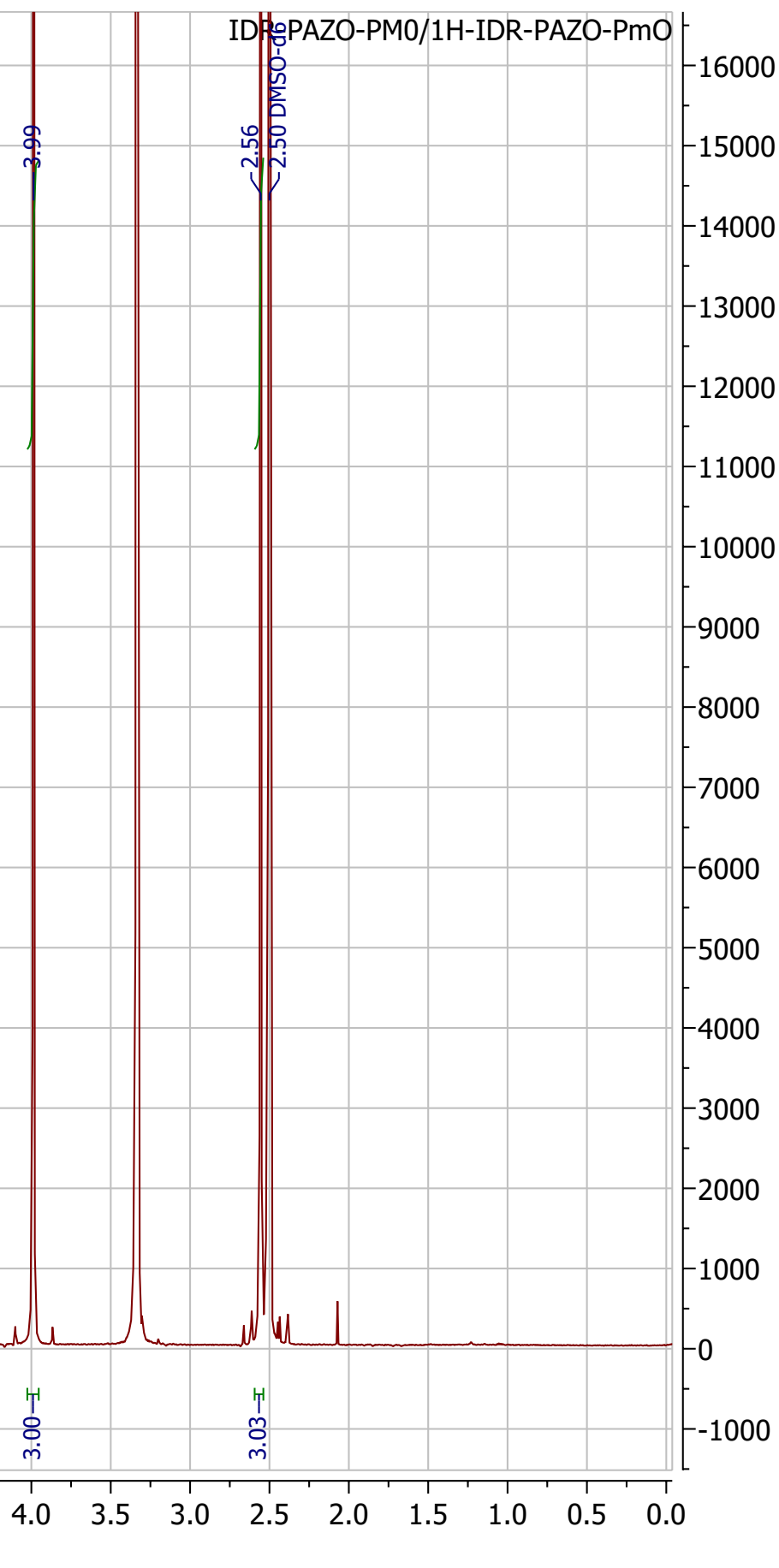

Fig. $65{ }^{1} \mathrm{H}-\mathrm{NMR}$ spectrum (300MHz, DMSO- $\left.\mathrm{d}_{6}\right)$ of 2-[(2,3-dimethylindazol-6-yl)amino] pyrimidin-4-ol 